\title{
Non uniform deposition of pMDI aerosol in a large volume spacer
}

\author{
by \\ Elizabeth Sprigge, B.Eng. \\ A thesis submitted to the \\ Faculty of Graduate and Postdoctoral Affairs \\ in partial fulfillment of the requirements for the degree of \\ Master of Applied Science \\ in \\ Mechanical Engineering \\ Ottawa-Carleton Institute for Mechanical and Aerospace Engineering \\ Department of Mechanical Engineering \\ Carleton University \\ Ottawa, Ontario \\ January, 2014 \\ (c) Copyright \\ Elizabeth Sprigge, 2014
}




\begin{abstract}
Chronic lower respiratory tract diseases have been cited as the fourth leading cause of death in Canada in both 2000 and 2009 [1]. Pressurised metered dose inhalers (pMDIs) administer medication for patients suffering from these disorders, in particular for asthma. Medication released from a pMDI can be difficult to inhale directly, and add-on devices have been designed to aid delivery.

While add-on devices, or spacers, increase the percentage of medication that reaches the patient, medication also deposits on the walls of the device.

The deposition of medication within a large volume spacer is studied, using spectrophotometry for experiments and computational fluid dynamics, implementing mean-flow and turbulent tracking of particles. Regions of deposition are of interest, as well as the way that the deposition varied for different inhalation flow rates. The aim is to determine optimal inhalation flow rate as well as studying the cause of the non-symmetrical deposition.
\end{abstract}




\section{Acknowledgments}

Firstly, I would like to thank Professor Matida for the opportunity to work on this project; I am grateful for his extensive help and guidance throughout this research and for always being available and able to answer any and all of my questions. I'd also like to thank Sandra Fiset for the work that she performed before me and for being such a great conference partner. I have been honoured and delighted to work with such knowledgeable and interesting people.

From the department, I express thanks to Professor Yaras for his advice regarding research, Bruce Johnston and Neil McFadyen for their IT support, Owen Roberts for helping me build things, David Raude and Steve Truttman for the equipment I borrowed, and everyone in the MAE office. These thanks are extended to the other 6th floor Canal Building students, especially Prameela Karumanchi, for sharing their conversation and ideas while I have been here, and everyone else who has made my Carleton experience what it has been.

I'd also like to thank Simon Blades, Ela Yuregir and my family; my grandparents for giving me a sense of perspective, my dad for everything he's taught me and Martha for being a great sister. Finally, I'd like to express how much I appreciate my mum, for her patience, her proof reading and for being there to "hold my fin". 


\section{Table of Contents}

Abstract $\quad$ ii

Acknowledgments

Table of Contents $\quad$ iv

List of Tables $\quad$ vii

List of Figures $\quad$ viii

Nomenclature $\quad x$

1 Introduction $\quad 1$

1.1 Thesis Statement . . . . . . . . . . . . . . . . . . . . . . . 1

1.2 The Respiratory System . . . . . . . . . . . . . . . . . . 2

1.2 .1 Conducting zone . . . . . . . . . . . . . . 3

1.2 .2 Respiratory Zone . . . . . . . . . . . . . . . . . . . . . 4

1.3 Lung Capacities . . . . . . . . . . . . . . . . . . . . . . . 5

1.3 .1 Gas Exchange . . . . . . . . . . . . . . 6

1.3.2 Resistance to breathing . . . . . . . . . . . . . . . . . 7

1.4 Lung Mechanics . . . . . . . . . . . . . . . . . . . . . . . 8

1.5 Gas Flow in Lungs . . . . . . . . . . . . . . . . . . . . . 9

1.5.1 Inspiratory flow rates . . . . . . . . . . . . . . . . 9

1.5.2 Respirable particles . . . . . . . . . . . . . . . 10

1.6 Lower Respiratory Diseases . . . . . . . . . . . . . . . . . . 11

1.6.1 Chronic Obstructive Respiratory Disease . . . . . . . . . . 11

1.6 .2 Bronchitis . . . . . . . . . . . . . . . . . . . . . 12

1.6 .3 Asthma . . . . . . . . . . . . . . . . . . 12

2 Background and Literature Review $\quad 14$

2.1 Prophylactic Medication . . . . . . . . . . . . . . . . 15

2.1.1 Corticosteroids . . . . . . . . . . . . . . 15

2.1.2 Long-Acting Bronchodilators . . . . . . . . . . . . . . 16

2.1 .3 Combination Medication . . . . . . . . . . . . . . . 17 
2.1.4 Alternative medications . . . . . . . . . . . . . . . 17

2.2 Reliever Medication . . . . . . . . . . . . . . . . . . . . 18

2.3 Salbutamol Sulphate . . . . . . . . . . . . . . . . . . . . . . . . . 19

2.3.1 Chemical Structure . . . . . . . . . . . . . . . . . . . . 19

2.3.2 Properties . . . . . . . . . . . . . . . . . . . . . . 19

2.4 Methods of Delivery . . . . . . . . . . . . . . . . . . . 21

2.4 .1 Nebulisers . . . . . . . . . . . . . . . . . . . . . . . . 21

2.4 .2 Dry Powder Inhalers . . . . . . . . . . . . . . . . . . . . 23

2.4 .3 Pressurised Metered Dose Inhalers _. . . . . . . . . . . 25

2.4 .4 Propellants . . . . . . . . . . . . . . . . 27

2.4 .5 Limitations . . . . . . . . . . . . . . . . . . . . 28

2.4 .6 Nozzle diameter . . . . . . . . . . . . . . . . . . . . . . . . . 30

2.4 .7 Spray properties . . . . . . . . . . . . . . . 33

2.4.8 Particle distribution of aerosol sprays . . . . . . . . . . . 36

2.5 Add-on Devices . . . . . . . . . . . . . . . . . . . . . . . . 38

2.5 .1 Open-Tube Spacers . . . . . . . . . . . . . . . . . . . . . . . . . 39

2.5.2 Reverse Flow Devices . . . . . . . . . . . . . . . . . . . . . 39

2.5 .3 Holding Chambers . . . . . . . . . . . . . . . . . 41

2.6 Add-on device studies $\ldots \ldots \ldots \ldots$

2.7 Inspiratory flow rate $\ldots \ldots \ldots \ldots$

3 Methodology 53

3.1 Experimental Description $\ldots \ldots \ldots \ldots \ldots \ldots$

3.2 Equipment . . . . . . . . . . . . . . . . . . . . . 55

3.2 .1 Flow Meter . . . . . . . . . . . . . . . . 55

3.2 .2 Pressure Transducer . . . . . . . . . . . . . . . . 55

3.2 .3 UV Spectrophotometer . . . . . . . . . . . . . . . . 56

3.2 .4 Salbutamol Sulphate . . . . . . . . . . . . . . . . . 60

$3.2 .520 \%$ Ethanol Solution . . . . . . . . . . . . . . . 60

3.3 Experimental Set up . . . . . . . . . . . . . . . . . . . 60

3.4 Experimental Procedure . . . . . . . . . . . . . . . . . . . . . . . 63

3.5 Wash Procedure . . . . . . . . . . . . . . . . . . . . 64

3.6 Calibration . . . . . . . . . . . . . . . . . . . . . 65

3.7 Deposition Measurements . . . . . . . . . . . . . . . . 66

4 Numerical methods $\quad 67$

4.1 Geometry . . . . . . . . . . . . . . . . 67

4.2 Boundary Conditions . . . . . . . . . . . . . . . . . . . . 68

4.3 Navier Stokes Equations . . . . . . . . . . . . . . . . . . . . . 69

4.4 Particle tracking . . . . . . . . . . . . . . . . . . 73

4.4 .1 Particle Size Distribution . . . . . . . . . . . . . . . . . 74

4.4 .2 Grid Convergence studies . . . . . . . . . . . . . . . 74 
$\begin{array}{lll}5 & \text { Results } & 77\end{array}$

5.1 Experimental results . . . . . . . . . . . . . . . . 77

5.1.1 Beer Lambert Calibration . . . . . . . . . . . . . . . 78

5.1.2 Deposition Measurements . . . . . . . . . . . . . . . 80

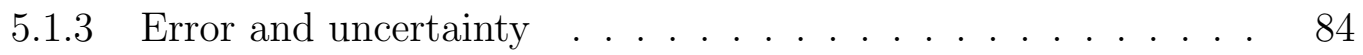

5.2 Numerical results . . . . . . . . . . . . . . . . . . . . . . . 87

5.2.1 Grid Convergence studies . . . . . . . . . . . . . . . 88

5.2 .2 Simulation results . . . . . . . . . . . . . . . . . . . 90

5.2 .3 Velocity in the spacer . . . . . . . . . . . . . . . . 90

5.2.4 Particle tracking . . . . . . . . . . . . . . . . . 94

5.2.5 Particle size distribution . . . . . . . . . . . . . . . . . 98

6 Discussions, Conclusions and Future Work 100

6.1 Discussions and conclusions . . . . . . . . . . . . . . . 101

6.2 Future Work . . . . . . . . . . . . . . . . . . . 101

$\begin{array}{ll}\text { List of References } & 104\end{array}$

$\begin{array}{ll}\text { Appendix A Experimental results } & 111\end{array}$

A.1 Deposition results . . . . . . . . . . . . . . . . . . . 111

A.2 Calibration results . . . . . . . . . . . . . . . . 113

$\begin{array}{lll}\text { Appendix B Uncertainty in measurements } & 114\end{array}$ 


\section{List of Tables}

2.1 Properties of salbutamol from a pMDI (adapted from Smyth) . . . . 20

2.2 Comparison of different spray conditions (adapted from Kleinstreuer et al.) . . . . . . . . . . . . . . . . . . . . 33

2.3 Parameters for Rosin-Rammler (adapted from Oliviera et al.) . . . . . 38

2.4 Characteristics of spacer devices tested (adapted from Barry et al.) . 46

4.1 Flow rate and mass flow outlets . . . . . . . . . . . . . . . 69

5.1 Mass of 100 actuations of Teva Novopharm Salbutamol . . . . . . . . 78

5.2 Measurement errors . . . . . . . . . . . . . . . . . . . . . 87

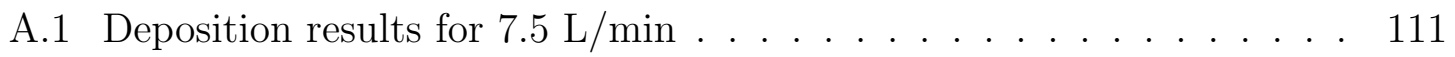

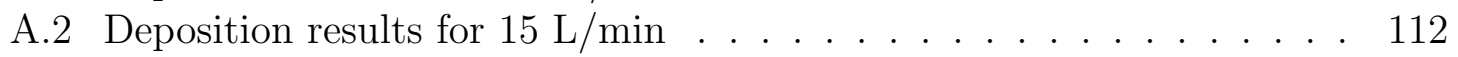

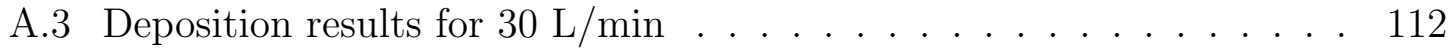

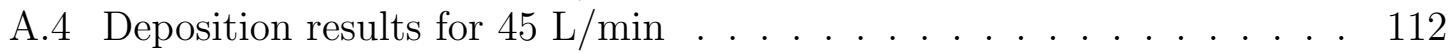

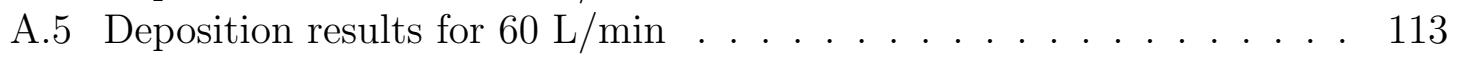

A.6 Absorbance measurements for Beer Lambert calibration curve . . . . 113

B.1 Proximal half of spacer . . . . . . . . . . . . . . . . . 114

B.2 Distal half of spacer . . . . . . . . . . . . . . . . . . . . . . . . . . . . . . . . . . . . . . 114

B.3 Upper half of spacer . . . . . . . . . . . . . . . . . . . . . . . . . . . . . . . . . . 115

B.4 Lower half of spacer . . . . . . . . . . . . . . . . . 115 


\section{List of Figures}

1.1 Respiratory system (adapted from Herman et al.) . . . . . . . . . . . 2

1.2 Lung capacities (adapted from West) . . . . . . . . . . . . . 5

2.1 Nebuliser . . . . . . . . . . . . . . . . . . . . . . 22

2.2 Dry Powder Inhalers . . . . . . . . . . . . . . . . . . . . . . . . . . . . . . . . . . . 23

2.3 TEVA Novopharm salbutamol sulphate pMDI . . . . . . . . . . 25

2.4 Diagram of actuator . . . . . . . . . . . . . . . 26

2.5 Effect of propellant on areas of deposition (adapted from Newman et al. 31

2.6 Effect of nozzle diameter on area at which medication will deposit (adapted from Newman et al.) . . . . . . . . . . . . . . . . 32

2.7 Temporal evolution of the velocity of the spray emitted from a Ventolinbrand pMDI (adapted from Crosland et al.) . . . . . . . . . . . . 34

2.8 Reverse flow spacers . . . . . . . . . . . . . . . . . . . . 40

2.9 Different volume holding chambers . . . . . . . . . . . . . . . . 40

2.10 Allen and Hanbury's Large Volume Spacer . . . . . . . . . . . . . . . 42

2.11 Effect of spacer length on the particle sizes that will reach patient (adapted from Bisgaard et al.) . . . . . . . . . . . . . 44

2.12 Effect of spacer diameter on the particle sizes that will reach patient (adapted from Newman) . . . . . . . . . . . . . . . 44

2.13 Effect of spacer volume on the amount of particles less than $5 \mathrm{mcm}$, adapted from (Barry et al.) . . . . . . . . . . . . . . 45

2.14 Salbutamol deposition for different spacers (adapted from Barry et al.) 47

2.15 Salmeterol dose to patient using different commercially available spacers (adapted from Barry et al.) . . . . . . . . . . . . . . . . 48

2.16 Areas of deposition with fast and slow inhalation rates (adapted from Newman et al.) . . . . . . . . . . . . . . . . . . . . . 49

3.1 How UV works . . . . . . . . . . . . . . . . . . . . . . . 58

3.2 Schematic of experiment . . . . . . . . . . . . . . . . 61

3.3 Spacer replica in four quadrants . . . . . . . . . . . . . . . . 61

3.4 Wooden holder for spacer with pressure transducer attached . . . . . 62

3.5 Experimental Set Up . . . . . . . . . . . . . . . . . 63

3.6 Respirgard $\mathrm{II}^{\mathrm{TM}}$ filter . . . . . . . . . . . . . . . . . . . . 65

4.1 Geometry made in ICEM by ANSYS. . . . . . . . . . . . . . . 68

4.2 Areas of interest for grid convergence studies . . . . . . . . . . . 76

5.1 Beer-Lambert calibration curve . . . . . . . . . . . . . . . . . . 79 
5.2 Percentage of spacer deposition . . . . . . . . . . . . . . 81

5.3 Upper and lower deposition . . . . . . . . . . . . . . . . . . . . . . . . . . . . . . 82

5.4 Proximal and distal deposition . . . . . . . . . . . . . . . . . . 83

5.5 Velocity along x-axis . . . . . . . . . . . . . . . . 88

5.6 Velocity along y-axis . . . . . . . . . . . . . . . . . . . 89

5.7 Velocity along z-axis . . . . . . . . . . . . . . . . 89

5.6 Fluctuating spray angle in time . . . . . . . . . . . . . . . . . 92

5.7 Velocity through spacer . . . . . . . . . . . . . . . . . . . . 93

5.8 Turbulent kinetic energy during actuation . . . . . . . . . . . . . 93

5.9 Mean flow particle tracking for $30 \mathrm{~L} / \mathrm{min}$ flow . . . . . . . . . . . . . 94

5.10 Side view for article deposition for $30 \mathrm{~L} / \mathrm{min}$ flow . . . . . . . . . . 95

5.11 Turbulent particle tracking for $30 \mathrm{~L} / \mathrm{min}$ flow . . . . . . . . . . . 95

5.12 Comparison between turbulent and meanflow tracking in ANSYS CFX 96

5.13 Mean flow tracking deposition results (CFD) . . . . . . . . . . . . 97

5.14 Experimental results for deposition . . . . . . . . . . . . . . 97

5.15 Comparison of spray angles . . . . . . . . . . . . . . . . . . 98

5.16 Comparison of uniform diameter and diameter distribution with experimental results . . . . . . . . . . . . . . . . . . . . . . 99

5.17 Particle diameter distribution . . . . . . . . . . . . . . . . . . . 99 


\section{Nomenclature}

\begin{tabular}{|c|c|c|}
\hline Symbols & Definition & Units \\
\hline $\mathrm{A}$ & Absorbance & - \\
\hline $\mathrm{a}$ & Constant of proportionality for pressure & - \\
\hline $\mathrm{b}$ & Path length, spectrophotometry & centimeters $(\mathrm{cm})$ \\
\hline $\mathrm{B}$ & Bias & - \\
\hline $\mathrm{C}_{p}$ & Specific heat capacity & $\mathrm{J} / \mathrm{K}$ \\
\hline $\mathrm{C}_{5}$ & Constant of proportionality & - \\
\hline $\mathrm{C}_{\mu}$ & Constant & - \\
\hline $\mathrm{c}$ & Concentration & $\mu \mathrm{g} / \mathrm{ml}$ \\
\hline $\mathrm{d}$ & Diameter & $\mathrm{m}$ \\
\hline $\mathrm{F}_{1}$ & Blending function & - \\
\hline $\mathrm{F}_{2}$ & Blending function & - \\
\hline $\mathrm{k}$ & Kinetic energy & $\mathrm{J}$ \\
\hline $\mathrm{k}_{1}$ & Calibration coefficient & - \\
\hline $\mathrm{L}$ & Length & $\mathrm{m}$ \\
\hline $\mathrm{m}$ & Mass & $\mathrm{g}$ \\
\hline $\mathrm{n}$ & Constant & - \\
\hline $\mathrm{P}$ & Pressure & $\mathrm{Pa}$ \\
\hline $\mathrm{Pe}$ & Pectlet number & - \\
\hline q & Quality flow parameter & - \\
\hline $\mathrm{q}_{e}$ & Size distribution parameter & \\
\hline $\mathrm{R}$ & Rosin-Rammler & - \\
\hline $\operatorname{Re}$ & Reynolds number & - \\
\hline $\mathrm{T}$ & Temperature & ${ }^{\circ} \mathrm{C}$ \\
\hline
\end{tabular}




\begin{tabular}{lll}
$\mathrm{U}$ & Uncertainty & - \\
$\mathrm{t}$ & time & $\mathrm{s}$ \\
$\mathrm{X}$ & Size constant & - \\
Directional & Definition & Units \\
\hline \hline $\mathrm{U}, \mathrm{V}, \mathrm{W}$ & Directional velocities & $\mathrm{m} / \mathrm{s}$ \\
$\mathrm{u}, \mathrm{v}, \mathrm{w}$ & Instantaneous directional velocities & $\mathrm{m} / \mathrm{s}$ \\
$\mathrm{x}, \mathrm{y}, \mathrm{z}$ & Direction along axis & - \\
$\mathrm{i}, \mathrm{j}, \mathrm{k}$ & Directional subscripts & - \\
\hline
\end{tabular}

\begin{tabular}{lll}
\hline Greek symbols & Definition & Units \\
\hline \hline$\alpha$ & Angle of spray & degrees \\
$\beta_{1}$ & SST Constant & - \\
$\beta^{*}$ & SST Constant & - \\
$\delta_{i j}$ & Kronecker delta & - \\
$\epsilon$ & Extenction coefficient of solvent & - \\
$\varepsilon$ & Dissipation & - \\
$\kappa$ & Diffusivity & $\mathrm{cm}{ }^{2}$ \\
$\gamma$ & SST Constant & - \\
$\lambda$ & Wavelength & $\mathrm{m}$ \\
$\mu$ & Turbulent viscosity & $\mathrm{kg} / \mathrm{sm}$ \\
$\nu$ & Viscosity & $\mathrm{kg} / \mathrm{sm}$ \\
$\rho$ & Density & $\mathrm{kg} / \mathrm{m}^{3}$ \\
$\sigma_{k 1}$ & SST Constant & - \\
$\sigma_{\omega 1}$ & SST Constant & - \\
$\tau$ & Transmittance & - \\
$\tau_{p}$ & Particle relaxation time & $\mathrm{t}$ \\
\hline
\end{tabular}




\begin{tabular}{ll}
\hline Subscripts & Definition \\
\hline \hline 0 & Incident \\
0.5 & Mass median \\
$\mathrm{A}$ & Atmospheric \\
$\mathrm{c}$ & Convective \\
$\mathrm{d}$ & Diffusive \\
$\mathrm{e}$ & Expansion chamber \\
$\mathrm{i}$ & Initial \\
$\mathrm{t}$ & Transmitted \\
\hline
\end{tabular}

\begin{tabular}{ll}
\hline Acronyms & Definition \\
\hline \hline ABS & Acrylonitrile butadiene styrene \\
APS & Aerodynamic particle sizer \\
BST & New baseline model \\
CAN & Controller area network \\
CFC & Chlorofluorocarbon \\
CFD & Computational fluid dynamics \\
COPD & Chronic obstructive pulmonary disorder \\
DF & Deposition fraction \\
DPI & Dry powder inhaler \\
FEV & Forced expiratory volume \\
FIF & Forced inspiratory flow \\
FRC & Functional residual capacity \\
FVC & Functional vital capacity \\
HFA & Hydrofluoroalkane \\
IC & Inspiratory capacity \\
LABA & Long acting beta agonist \\
LES & Large Eddy-simulation
\end{tabular}




\begin{tabular}{ll} 
MDI & Metered dose inhaler \\
MMAD & Mass mean average diameter \\
MMD & Mass mean diameter \\
MSLI & Multi-stage liquid impinger \\
pMDI & pressurized metered dose inhaler \\
PDPA & Phase-Doppler particle analysis \\
PIV & Particle image velocimetry \\
RANS & Reynolds averaged Navier-Stokes \\
RV & Residual volume \\
SST & Shear stress transport \\
TLC & Total lung capacity \\
TV & Total volume \\
URANS & Unsteady Reynolds averaged Navier-Stokes \\
UV & Ultra violet \\
VC & Vital capacity \\
\hline
\end{tabular}




\section{Chapter 1}

\section{Introduction}

\subsection{Thesis Statement}

Large-volume spacer devices are used with pressurised metered dose inhalers (pMDIs) to enhance the delivery of aerosol administered medication. Though spacer devices have been proven to enhance the amount of medication that will reach a patient,

some medication is lost through deposition occurring on the spacer walls [2-8]. The aim of this thesis is to determine the areas at which this deposition will occur, and the causes of deposition, within a large volume $(750 \mathrm{ml})$ spacer. Considering the Volumatic ${ }^{\mathrm{TM}}$ spacer (Allen and Handburys, UK) in four quadrants, the deposition is quantified experimentally and modelled numerically in order to determine whether deposition occurs due to inertial impaction or due to turbulent flow, and to determine if the deposition is asymmetric or symmetric.

This thesis will also study the effects of using different inhalation flow rates on the amount of medication that will reach a patient using a large-volume spacer, and will assess the effect that these different rates have on the deposition within the spacer itself. For a patient suffering from acute asthma, it is important for a large amount of medication to reach the lungs, and therefore this research intends to specify the 
optimal

Medication administered as an aerosol acts primarily on and through the respiratory system. This chapter provides a brief description of the respiratory system and its components, mechanics and potential diseases that can affect the lungs.

\subsection{The Respiratory System}

The respiratory system's main function is to provide oxygen to the tissues of the body and to eliminate carbon dioxide. It consists of two main parts parts; the "conduction" zone and the "respiratory exchange" zone [9].

The components of the respiratory system are shown in Figure 1.1.

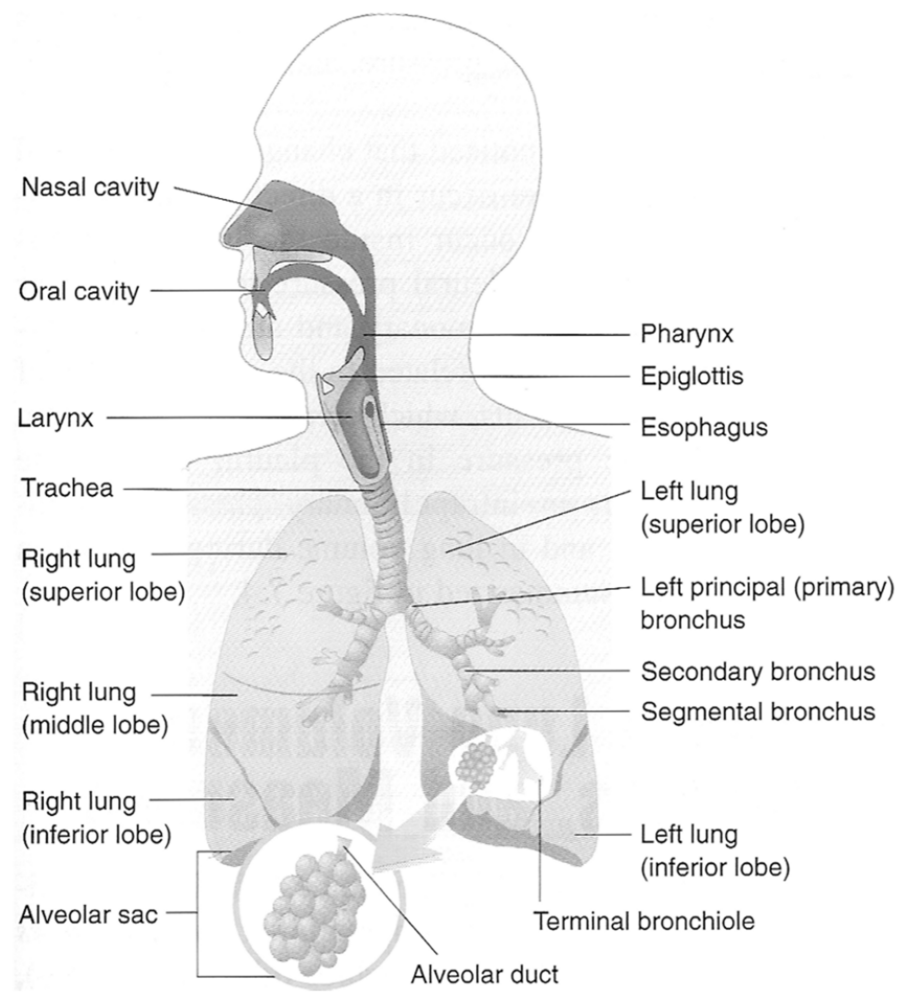

Figure 1.1: Respiratory system, adapted from [10] 


\subsubsection{Conducting zone}

The conducting zone of the respiratory system acts to transfer oxygen in the outside air to reach the lower regions of the lungs where gas exchange can occur. The conduction zone includes the mouth and nose, the pharynx, the trachea and the bronchi and bronchioles, and provides filtration, humidification and laminarisation of the air that is breathed in.

The mouth and nose are inlets to the respiratory system and connect at the laryngopharynx, via the oro- and naso-pharynx [11]. Air inhaled through the nose is heated by the nasal cavities and is filtered by the small hairs and mucous present in the nostrils. The majority of large inhaled particles will be stopped by this filtration mechanism, or will impact on the walls of the nasopharynx due to the turbulent flow. When breathing through the mouth, this filtration and warming does not occur and the resistance to airflow is halved, making mouth breathing a more effective route of aerosol medication administration.

The laryngopharynx connects to the larynx which houses the voice box and prevents food from entering the trachea; a tube which is around $12 \mathrm{~cm}$ in length, made up of cartilage in C-shaped sections that ensure that the passage remains open [12]. The upper half of the trachea is outside of the rib cage and the lower is intra-thoracic. At the fifth thoracic vertebra the trachea bifurcates into the left and right bronchus, where the right main bronchus makes a shallower angle to the left because of the asymmetry of the lungs. As room is required to accommodate the heart, the left lung is made up of two lobes, where the right has three [13]. Because of this the right lung has $10 \%$ higher volume, making it more susceptible to infections. The different lengths and angles of the bronchi may also have an impact on the deposition within the lungs.

The branching that occurs at the base of the trachea continues throughout the 
lungs, and the bronchi split further to become bronchioles into parent and daughter bronchiole branches [14]. There are around 23 bifurcations from the trachea to the alveoli sacs, with the number of branches at each generation following a Weibel distribution [14]. The upper 16 generations are considered to be the conducting zone and the lower seven, part of the respiratory zone.

\subsubsection{Respiratory Zone}

In the respiratory zone of the airways, gas transport occurs due to diffusion and respiratory bronchioles have increasingly small diameters (from $0.3 \mathrm{~mm}$ to $0.6 \mathrm{~mm}$ ). As well as the layer of epithelium present on the surface of all the airways, the final stage of branching (called the "terminal bronchoiles") are also covered by alveolar epithelium, which allows oxygen to diffuse from the lungs to the blood. Attached to the terminal bronchioles are the alveolar ducts which are small, polyhedral sacs which provide a large surface area through which air can diffuse, as the walls are only one cell thick. It is important that the alveoli contain small amounts of air even during exhalation so that the lungs retain a residual volume, as a collapsed alveoli requires a large amount of pressure to re-inflate. To prevent this from occurring the alveolar walls are covered with a lipid substance called a surfactant, which will maintain their stability to remain open. It is also important for the airways prior to the alveoli to remain open, in order for the lower regions of the lung to be reached by the air inhaled with each breath [15].

Before reaching the alveolar sacs, all bronchi and bronchioles have a thin lining of muscle. This muscle assists with breathing mechanism and holds the airways open. These muscles are affected by respiratory disorders which involve inflammation. Nerves and receptors run alongside the muscles which, when stimulated, will cause the smooth muscle in the bronchiole walls to contract or go into spasm, causing the 
diameter of the airways to change in such a way that there is increased resistance to breathing, and increased occurrence of inflammation [15].

\section{$1.3 \quad$ Lung Capacities}

Lung volumes and the compliance of lungs and chest wall (how much the lungs and chest wall can stretch) dictate much of the behaviour of the respiratory system as they impact how much air is available at points of the respiratory cycle for gas exchange. The total lung capacity (TLC) is broken into different subsections which have significance in determining how the lungs are working, and is shown in Figure 1.2 .

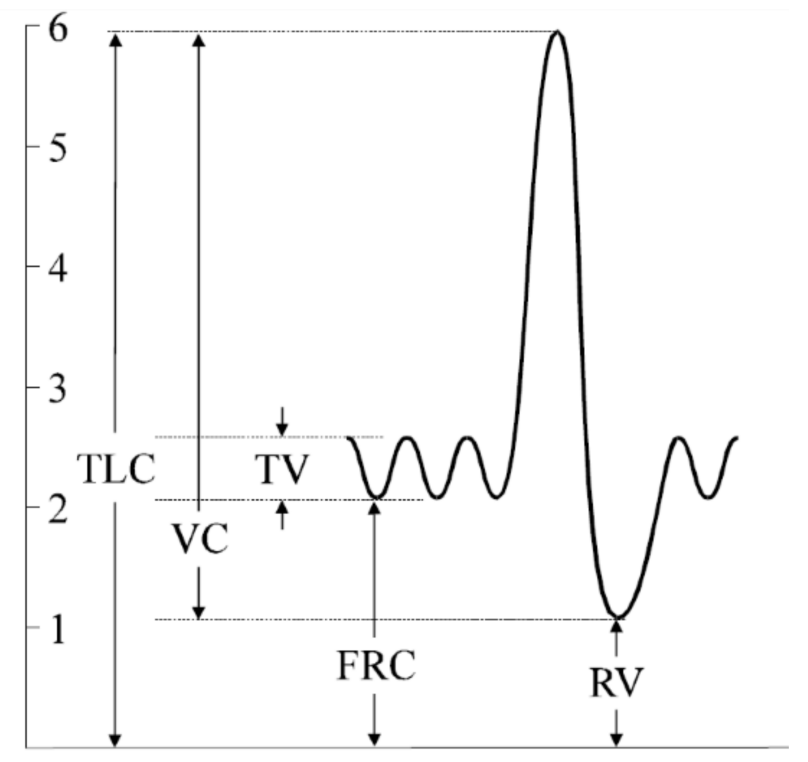

Figure 1.2: Lung capacities (adapted from [15])

The lungs have a residual volume (RV) of air that is always present. This cannot be expelled even with maximum exhalation, and is left to ensure that the lungs and the small alveolar sacs do not collapse. During normal breathing there is a also a functional residual capacity (FRC), which is the volume of lungs that remains in the 
lungs after breathing out, ensuring that the alveoli remain open. This air acts as a buffer for the lungs as it will increase the average volume, reducing the amount of work that is required for each inhaled breath. The air in the FRC is replaced with each new breath, as it is in this air that gas exchange occurs. During normal respiration the tidal volume (TV) is the amount of air that will be taken in, and the inspiratory capacity (IC) is the maximum volume of air that can be inspired from a resting respiratory level. The vital capacity $(\mathrm{VC})$ is the volume of air that can be exhaled after inhalation, and can be measured using spirometry. The forced vital capacity (FVC) can also be estimated using the spirometer. From spirometry a flow-volume loop can be created, as it tests the forced expiratory volume (FEV, or $\mathrm{FEV}_{1}$ for the volume inhaled in one second of forced exhalation). From this the forced inspiratory flow (FIF) can be calculated, being the flow rate which is achieved when a patient breathes in as deeply as possible, which is important for some aerosol

medication devices. $\mathrm{FEV}_{1}$ and $\frac{\mathrm{FEV}_{1}}{\mathrm{FVC}}$ can be used to help diagnose asthma and other respiratory disorders [15].

\subsubsection{Gas Exchange}

Gas exchange through the respiratory system occurs either due to convection or diffusion. The determining factor of the type of gas exchange that will occur between two gases is related to the Peclet number, which is the ratio of the diffusive time for transport to the convective time for transport, as shown below [14]. For large Peclet numbers, diffusion can be neglected.

$$
t_{c}=\frac{L}{U}, t_{d}=\frac{L^{2}}{\kappa}, P e=\frac{t_{d}}{t_{c}}
$$


Where;

$t_{c}$ is the time for conduction to provide effective transport

$t_{d}$ is the time for diffusion to provide effective transport

$L$ is the airway length of the generation

$U$ is the average flow speed of the gas

$\kappa$ is the diffusivity of the gas pair, which has a value of $0.22 \mathrm{~cm}^{2} / \mathrm{s}$ for oxygen to air.

In the upper airways the gas transport will be mainly due to convective transport, and in the lower generations the value of Pe will decrease and diffusion will be the dominant form of gas transport. At the alveoli, gas exchange across the membrane of the lungs will occur and, due to the large surface area, the flow will be solely diffusive.

\subsubsection{Resistance to breathing}

Asthma is a disease that involves increased sensitivity of the muscles in the airways. In acute asthma the airways can constrict due to either bronchospasm, inflammation, or both. When this occurs there is an increased resistance to airflow, resulting in patients being unable to take in new air and expel old air. Hyperinflation of the lungs occurs due to the difficulty in expelling the trapped air and the increase in airway pressure [16]. As the pressure increases in the lungs, the work done in each breath increases proportionally (as work done in breathing is the product of pressure and volume), making it necessary for the respiratory muscles to work harder. Over a prolonged period of time the muscles will become exhausted and gas exchange mechanisms will be impaired, resulting in respiratory failure. 


\subsection{Lung Mechanics}

Inhalation and expiration occur due to pressure differences between air in the alveolar sacs and the atmosphere. The number of muscles involved in breathing depends on the force used; for "quiet" breathing the diaphragm will lower and external intercostal muscles will raise the ribs upwards and outwards, creating a pressure difference of around $3 \mathrm{mmHg}$ and therefore causing air to be drawn into the lungs and the volume of the lungs to increase. With increased force in inhalation, an increased number of muscles will become involved, helping to further raise the thoracic cage and reducing the resistance in the upper airways [16].

The thoracic cage is made up of the ribs, the diaphragm, the sternum and the intercostal muscles. The diaphragm provides $75 \%$ of the power required for quiet breathing, where the remaining $25 \%$ is due to ribcage movement [12]. Pleural fluid between the lungs and ribcage both acts as a lubricant and causes the two to stick together, allowing unrestricted movement during breathing, rather than any muscle ever acting on the lungs directly.

Exhalation is a passive process whereby the diaphragm and the intercostal muscles relax, so that the diaphragm rises and the ribs move downwards. With this, the volume of the lungs will' decrease, forcing air out of the mouth or nose. More muscles, such as the sternocleidomastoid muscles, become involved with increasing force in inhalation or increased work of breathing, and with increased force the ratio of time for inhalation to time for exhalation will decrease. Hyperventilation and exercise may induce this increased force.

As well as the major muscle groups, muscles in the linings of the airway walls are also used in respiration. Contraction and expansion of the airways due to these muscles assists in increasing and decreasing lung volumes, and in the flow of air at 
diffusive levels.

\subsection{Gas Flow in Lungs}

The flow of air in the lungs is dependent on a combination of the rate at which the air is inhaled and on the size of the airways that the air is passing through. The predominant method of flow varies for different sections of the lungs because of the large variation in airway diameter and because of the distance travelled since inhalation. Particles within the airflow will also be affected not only by the flow but also the size, as particles must be smaller than a certain diameter to reach different parts of the lungs.

\subsubsection{Inspiratory flow rates}

Inspiratory flow rates vary due to a number of reasons, such as lung volume, patient size and age, and respiratory health. Inspiratory flow rates are important when administering medication as an aerosol, or for inhalation therapy. Devices such as pMDIs, dry powder inhalers (DPIs) and nebulisers are affected by inspiratory flow rates as they determine the regions of the lungs that the aerosol will reach, the amount that will be taken in during one breath, and the turbulence that will be present in the flow. For some devices (most commonly DPIs) there is a certain rate at which inhalation must occur in order to actuate the device, requiring the patient to be able to achieve a certain FIF rate. Conversely, nebuliser treatment is dependent on the minute volume (the volume of air inhaled in a minute), as the medication is administered as a constant flow.

For the majority of the respiratory airways the airflow is transitional to laminar, as mentioned in Section 1.2.1. In the lower respiratory tract, further down the 
bronchial tree, the Reynolds number is low and flow is mainly laminar, interrupted at bifurcations due to flow separating from the walls and because airways may have different impedances (resistances to flow due to the pressure in each region) [17]. In quiet breathing turbulence is mainly confined to the trachea but with high minute ventilation, flow through the bronchi may also be affected. Turbulence in the upper airways can cause impaction of aerosols on the airway walls, trapping inhaled medication before it reaches a point where it would have beneficial effect.

For inspiratory flow rates, the minute volume and the rate of ratio of inhalation to exhalation need to be taken into account, the I:E ratio. For an adult the average I:E ratio is $1: 2$, for children $1: 3$ and for asthmatics, due to the increased effort required to expel air, the ratio can increase to 1:6 during acute asthma. The minute volume is calculated using:

$$
\text { Minute volume }=(\text { Respiratory rate })(\mathrm{TV})
$$

For an adult male the average minute volume is $7500 \mathrm{ml}$ during quiet breathing, giving a inspiratory flow rate of $30 \mathrm{~L} / \mathrm{min}$. As TV is proportional to a person's weight these flow rates can be as low as $7.5 \mathrm{~L} / \mathrm{min}$ in a $25 \mathrm{~kg}$ child.

\subsubsection{Respirable particles}

Respirable particles are particles which can be inhaled into the terminal bronchioles and alveoli, and have an aerodynamic diameter of less than $10 \mu \mathrm{m}$. The aerodynamic diameter of a particle is the idealised diameter of a particle, if it were a sphere with uniform density. The aerodynamic diameter is used in simulations rather than the actual diameter, as the shapes of small particles can be irregular, so an idealised particle is used as an alternative that will behave identically to an actual particle. The size of inhaled respirable particles influences the region in which deposition will 
occur in the lungs due to the decreasing diameter of the airways. Deposition in the lungs occurs as a result of inertial impaction, sedimentation, diffusion and interception [18]. Inertial impaction is most common for larger particles which will deposit on the walls of the upper airways due to the irregularity in the flow. Particles with smaller aerodynamic diameters will penetrate further than the mouth and nose and are referred to as the fine particle fraction $(\mathrm{FPF})$ of an aerosol. The value for this is found to be $4.7 \mu \mathrm{m}[19]$, as this is the diameter of particle that is found to penetrate the lungs. Within the lungs impaction occurs due to particle size and sedimentation can occur when particles are stagnant in airways. As particle size decreases, more distal regions of the lungs will be reached up to a certain point. However particles that are too small will not affect any region of the lung as they will be exhaled [20]. The peripheral airways are affected by inflammation in respiratory diseases and therefore, it is the peripheral airways that should be targeted by the aerosol medication.

\subsection{Lower Respiratory Diseases}

Lower respiratory tract diseases, which include asthma, chronic obstructive respiratory disease (COPD) and bronchitis, are diseases which affect the conductive and gas exchange zones of the respiratory system. The affects of these diseases cause difficulty in breathing and airway inflammation.

\subsubsection{Chronic Obstructive Respiratory Disease}

COPD is a disease that usually presents itself in later life, and is predominantly a response to smoking. COPD increases airway resistance due to a number of factors; loss of elasticity in the airways, clogging of the airways due to the excess mucous

produced, and inflammation of the airway walls. Treatment of COPD includes using 
bronchodilators to reduce airway inflammation. As the disease is both restrictive and obstructive, the total lung capacity is reduced and thus additional treatment is required, such as pulmonary rehabilitation in order to learn how to use the reduced amount of available air or supplemental oxygen, where extra oxygen is supplied to the body through nasal prongs or face mask.

\subsubsection{Bronchitis}

Bronchitis is an infection, generally viral, that results in inflamed bronchi. It can be treated in the same way as other lower respiratory disorders because of the nature of its symptoms and the area that it affects, and can associated with COPD.

Bronchitis can be treated with medication such as inhaled salbutamol sulphate in order to relax the bronchi that have become inflamed. This treatment is taken through a puffer and will take effect shortly after administration, but acute (short term) bronchitis can also be left untreated and the patient will usually recover in a number of weeks.

\subsubsection{Asthma}

Asthma presents with chest tightness, wheezing, coughing and dyspnea (shortness of breath) due to increased resistance to airflow. The symptoms of acute asthma, or an asthma attack, are caused by airway hyper-reactivity, in which the bronchi and bronchioles will constrict, causing increased resistance to airflow. This hyperreactivity can be brought on by a number of factors, for example inhaling allergens or exercise, particularly in the cold. The bronchial hyper-reactivity is accompanied by an increase in mucous production and inflammation of the bronchi. As asthma is a disorder of increased airway resistance, treatment should be administered that will 
promote the dilation of the bronchi and bronchioles. A third of asthma sufferers will show symptoms before the age of ten and most cases will be diagnosed before the age of 40 [21].

\section{Allergic Asthma}

Allergic asthma has the same symptoms and effects as acute asthma in that the bronchioles become inflamed and constrict, causing a resistance to airflow and making it difficult to breathe. In allergic asthma these symptoms are triggered by an external factor and can be treated in the same way as acute asthma but other medication can also be taken in order to prevent the lungs from reacting to the inhaled allergen. Allergic asthma can also be treated with fewer medications in some cases, if the reaction can be stopped before presenting with symptoms of shortness of breath, as a result of inflamed bronchi. 


\section{Chapter 2}

\section{Background and Literature Review}

In this chapter the background of the present research will be discussed, with descriptions of the available medications for asthma treatment and the different delivery methods available, including the add-on devices which are used to enhance the delivery of aerosol medication.

This chapter will also discuss studies which have been carried out relating to these delivery methods.

To treat asthma a two-stage approach is taken, depending on the severity of the disease and the level of control that the patient has over their symptoms. For the treatment of the underlying causes of asthma prophylactic or preventative medications are taken. These include inhaled therapy as well as oral and intravenous options. Prophylactic medications address the causes of inflammation and excess mucous and are taken by asthmatics even when their asthma seems to be under control (which is when acute asthma attacks rarely occur). These medications will not have an effect directly after administration, but provide treatment that is longer lasting.

For immediate relief of acute asthma symptoms, bronchodilators are used. These relax the muscles that surround the airways and act quickly to allow the patient to breathe with less resistance, but cannot treat the underlying causes of inflammation. 


\subsection{Prophylactic Medication}

Prophylactic medications, also referred to as "controllers", prevent asthma attacks by treating the inflammation of the lungs that causes asthma, as well addressing the effects of allergens for people with allergic asthma.

There are a number of different medications available for prescription by a physician. These include steroids, bronchodilators and combination medication as the main options, as well as a number of anti-inflammatory medications that can be used to treat allergic asthma [22].

\subsubsection{Corticosteroids}

Corticosteroids are the most commonly prescribed preventative medication, and are the most effective for reducing inflammation of the airways. They act to reduce the swelling of the airways that occurs in an asthmatic's lungs, and can be administered orally, through injection, or by inhalation.

Oral, intravenous and intramuscular treatment provide high doses of corticosteroids systemically for severe cases of asthma. Generally this is not recommended for long-term use, and oral medication will only be given to a patient for a short period of time to treat problems with controlling the frequency of attacks. Due to the amount of steroids in oral medication, the side effects of long term treatment using higher doses far outweigh the advantages. Examples of tablet and liquid forms of oral corticosteroids are prednisone and prednisolone [22].

Inhaled corticosteroids are regularly prescribed to asthmatics as the main preventer medication of choice. Doses of medication are given in micrograms and include budesonide and fluticasone. They can be administered in aerosol or powder form depending on the device used. In this way, lower doses can be administered directly 
to the involved organ decreasing systemic side effects.

\section{Side Effects}

Corticosteroids cause side effects depending on the strength of the dose and the method of delivery. The major side effect is immuno suppression, making the patient more susceptible to infection [23]. If corticosteroids are inhaled they can cause oral thrush due to drug that remains within the mouth, which can be avoided if the patient rinses their mouth after using their inhaler. Oral and injected corticosteroids produce greater side effects, partly due to the increased dose that is administered using these methods. Side effects include mood changes, weight gain and osteoporosis. It is suggested that oral and injected methods are not taken for long periods of time because of this, though in some cases it is required for severe and badly controlled asthma [22].

\subsubsection{Long-Acting Bronchodilators}

Long-acting bronchodilators, also known as long-acting beta agonists (LABAs), are taken alongside corticosteroids for some patients with asthma. LABAs are said to enhance the way that the corticosteroids work, and will keep the airway dilated for up to twelve hours after administration. They are not prescribed without corticosteroids and examples include salmeterol and formoterol.

\section{Side Effects}

Long acting bronchodilators target beta 2 -receptors and because of this they can increase the chances of a patient experiencing tremors (a beta ${ }_{2}$-agonist will make muscle more sensitive to stimulation). Other side effects include anxiety, rashes and chest pain, as well as increased heart rate and arrhythmias [22]. 


\subsubsection{Combination Medication}

In order to simplify the use of preventative medication for an asthmatics, combination medications have been formulated so that the patient only needs to take one medication rather than two, if being treated with corticosteroids as well as LABAs. This is easier to use and gives the patient better control over their condition.

In combination medication, LABAs will widen the airway and steroid will act to reduce and prevent inflammation in the airways. The side effects of combination medication are a combination of the side effects that occur from the corticosteroid and LABA alone [24].

\subsubsection{Alternative medications}

Alternative methods of treatment include anti-leukotrienes that block a chemical reaction that causes airway inflammation and anti-immunoglobulin E therapy, which blocks immunoglobulin E, to prevent inflammation from occurring. Theophylline was the first medication that was used to treat asthma, and is still used if asthma disrupts sleep or for asthma that cannot be controlled by other medications. Theophylline has a number of side effects which is one of the reasons that it is not used as a regular method of treatment for asthma, for example increased heart rate and arrhythmia [24].

\section{Side Effects}

Alternative medications have a variety of side effects, dependent on which is taken. The commonly occurring side effects are upset stomach and heart burn, and theophylline can, on top of these, cause insomnia and can effect the patients mood. 


\subsection{Reliever Medication}

Reliever medications are used for short-term relief of asthma symptoms when the airways become inflamed. Asthma sufferers are prescribed reliever medication on top of the preventative treatment, and these relievers should not be used alone as they will not address the cause of inflammation but will solely provide relief from the symptoms. These short-acting medicines are either anticholinergics or inhaled beta ${ }_{2}$-agonists. The beta ${ }_{2}$-agonists target the beta ${ }_{2}$-adrenergic receptors in the bronchial muscles, relaxing them so that the bronchioles dilate and there is less airway resistance.

The medication that is available to be prescribed includes fenoterol, formoterol and salbutamol.

Salbutamol is a regularly chosen medication for prescription. It can be taken through inhalation or can be injected. Injection of salbutamol is for serious, life threatening attacks, and in these cases 5-10 $\mathrm{ml}$ of medication is administered. Tablets are also available for severe attacks where large amounts of medication are needed. Generally this is not required, and the beta ${ }_{2}$-agonist is self-administered through inhalation [24].

\section{Side Effects}

Reliever medication and LABAs have similar side effects, as they target the beta $2^{-}$ receptors. Because of this, for reliever medication, increased heart rate and tremor can sometimes occur, as well as restlessness. 


\subsection{Salbutamol Sulphate}

As described above, salbutamol sulphate is a commonly prescribed reliever medication in the treatment of asthma. It is a beta 2 -agonist and targets beta $_{2}$-adrenergic receptors which exist in the lungs, causing bronchodilation allowing patients to breathe more easily. It is used for the treatment of respiratory disorders, particularly for the quick relief from acute asthma (typically relief will be felt within 5 - 15 minutes of administration) [25].

\subsubsection{Chemical Structure}

The molecular formula of salbutamol sulphate is $\left(\mathrm{C}_{1} 3 \mathrm{H}_{2} 1 \mathrm{NO}_{3}\right)_{2} \cdot \mathrm{H}_{2} \mathrm{SO}_{4}$.

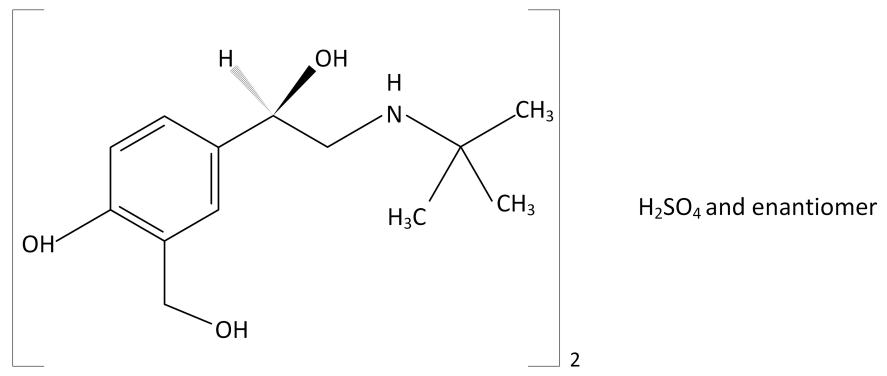

\subsubsection{Properties}

Salbutamol sulphate comes in the form of a white crystalline powder. It is soluble in water, chloroform and ether, and shows a clear negative cotton effect at 276 $280 \mathrm{~nm}$ [26] when dissolved in ethanol. The cotton effect shows the characteristic wavelength for a substance, according to its maximum optical rotatory dispersion or maximum circular dichroism (the difference between right and left hand circularly polarised light) [27]. 
Salbutamol sulphate targets beta 2 -adrenergic receptors in the bronchial muscle, and relaxes the muscle fibres and will therefore decreases the work of breathing. When a patient begins to show signs of acute asthma, 100 - 200 micrograms of salbutamol sulphate is taken, and this same dose can also be taken prior to exercise in order to avoid exercise-induced bronchospasm. The maximum dose of salbutamol sulphate in a day is eight sprays and if a patient starts needing to use this more than three times a week it is suggested that they see a physician.

While salbutamol sulphate can have side effects, causing tremors from inhalation, the doses that are given are small enough that if the drug is swallowed when inhaled in aerosol form, the liver will metabolise the active ingredients and side effects are not experienced.

Released from a pMDI, salbutamol sulphate has the following properties:

\begin{tabular}{cc}
\hline Property of salbutamol & Value \\
\hline \hline Density, $\mathrm{kg} / \mathrm{m}^{3}$ & 1230 \\
Actuation dose, $\mu \mathrm{g}$ & 100 \\
Actuation time $(\mathrm{s})$ & 0.1 \\
\hline
\end{tabular}

Table 2.1: Properties of salbutamol from a pMDI (adapted from Smyth [28])

\section{Particle Size}

Respirable particles, as described in Section 1.5.2 are particles under $10 \mu \mathrm{m}$ in diameter. Salbutamol sulphate has a mass mean aerodynamic diameter (MMAD) of $3.53 \mu \mathrm{m}$ when released from an actuator with a spacer, which is within the respirable range of particles that will reach the lungs [29]. The distribution of the particles sizes is entirely dependent on the method of aerosolisation, and probability density functions of particle size distribution vary depending on the device chosen. 


\subsection{Methods of Delivery}

There are a number of devices available to deliver aerosolised medication to the lung,of which there are three main categories of inhaler used. The device that is chosen to administer medication depends on the patient, the doctor and the illness. The devices are nebulisers, dry powder inhalers and metered dose inhalers.

\subsubsection{Nebulisers}

Nebulisers were the first devices used for aerosol drug delivery, and were introduced in the 1800s. These devices break up large molecules of medicine into a mist of smaller droplets, which is inhaled by a patient through a mouthpiece or face mask. The use of nebulisers for the treatment of asthma is declining as newer, less expensive devices are available, but they are still used if the prescribing physician suggests it, or if a patient requests it. Nebulisers are also still used for administration for illnesses other than asthma.

There are a variety of methods for breaking up the liquid into smaller droplets, which is why nebuliser treatment can be expensive. Ultrasonic waves is the most expensive method available, requiring an ultrasonically vibrating crystal to break the medication into droplets. Ultrasonic nebulisers are the smallest and most portable available, as they are self-contained, and are relatively quiet, unlike jet nebulisers. Jet nebulisers use either electrical or gas compressors to pass a stream of air or oxygen through the liquid medication, which will break it up into the small droplets needed for inhalation therapy. At the outlet of the compressor and medication, a mouthpiece or face mask is attached. As jet nebulisers use compressed gas or electricity, it is necessary for the patient to remain in one place with a power supply during their treatment, which is one factor that makes nebuliser use inconvenient. Due to their 
shape and size, jet nebulisers are less portable than other available therapies.

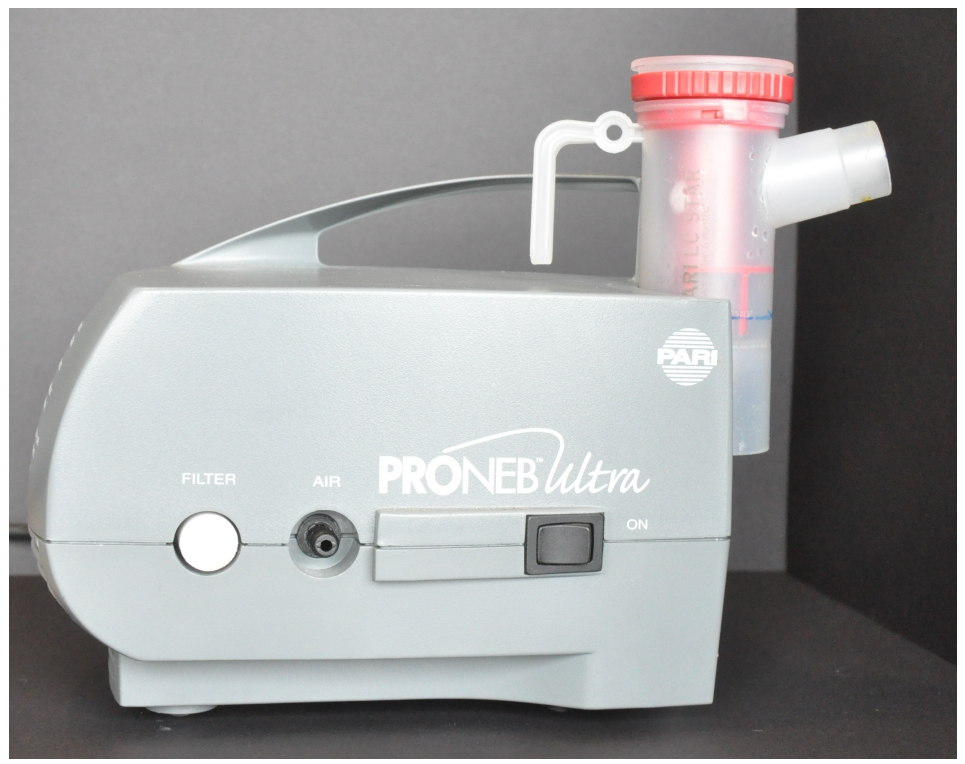

Figure 2.1: Nebuliser

The nebuliser, whether ultrasonic or jet, runs continuously while medication is being administered, which is usually for around 10 to 15 minutes. During this time the patient breathes normally through the face mask or mouthpiece. As inhalation only makes up one third of a breathing cycle, the majority of medication released is not inhaled unless a specially designed mouthpiece is used to control the release of medication. Some nebulisers allow the medication to drop back into the reservoir above the jet of gas in order to be re-administered. The design of the mouthpiece and connector also makes the nebuliser less efficient, as the tubing is connected with T-junction, a lot of medication will deposit on the walls of the plastic, rather than reaching the patient. While this decreases the efficiency of the device, it also results in wall impaction of larger particles in the aerosol, meaning that the non-respirable medication will not reach the mouth of the patient but smaller, respirable particles will. 
Nebuliser use is decreasing as other therapies are more convenient, less expensive and more efficient in treating asthma. However for patients with, for example, arthritis or who are particularly weak, nebulisers can still be the preferred method of delivery as they do not need to actuate by pushing a button, or by breathing at a high flow rate. They are also used regularly in hospitals where patients can only breathe passively, because of their design and the lack of requirement for a certain inspiratory flow rate in order for the treatment to be effective. Nebulisers are also beneficial for situations where large doses are required, but it has been found that using a large volume spacer with a puffer is also a plausible alternative [30].

Although they are no longer recommended and it is suggested that alternative therapies are tested prior to use of the nebuliser, some physicians and patients prefer their application and for this reason nebulisers can still be used in a domestic environment for the treatment of asthma.

\subsubsection{Dry Powder Inhalers}

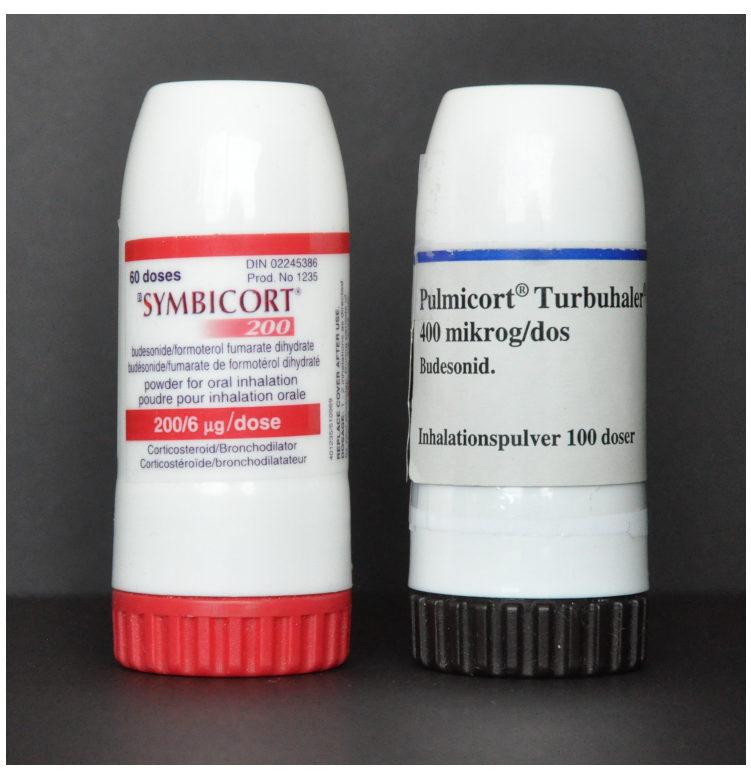

Figure 2.2: Dry Powder Inhalers 
DPIs are self-actuated devices for administering medication. There are a variety of types available which use medication stored in different ways: in blister packs which can be either be opened by the patient or pierced by a part of the device, or in reservoir devices, or the medication can be stored within the device itself (which requires a pre-metering part of the DPI design). Medication is in powder form and breath is the propelling force used to actuate the device. DPIs can be used for reliever medication such as salbutamol sulphate but are more common for use with preventative medication.

To use a DPI the patient inhales deeply at a rate between $30 \mathrm{~L} / \mathrm{min}$ and $90 \mathrm{~L} / \mathrm{min}$ [31]. Because the device is breath actuated, there is no requirement for the patient to be able to synchronise their breathing, which can be problematic for some patients. The method of actuation is also beneficial for patients with arthritic hands as it does not have an external trigger that needs pressing. The devices are also small, portable and inexpensive which makes them a preferred choice in treating asthma. DPIs have also become more popular since the Montreal Protocol came into effect in 1987. The Montreal Protocol banned the use of chlorofluorocarbons (CFCs) because of their negative effect on the ozone layer. pMDIs, until that point, had used CFCs as a propellant for the medication. Measures were taken to replace the CFCs with hydrofluoroalkanes (HFAs), but DPIs are still the most secure alternative as HFAs are also greenhouse gases, and though their impact on the environment is much smaller there is still a chance that in the future they will need to be replaced.

DPIs, although highly convenient, are not suitable for all asthmatics. The high inspiratory flow rate requirement can be problematic for younger patients and those suffering from COPD, or who have another condition which causes difficulty in breathing deeply. Without the deep breath, the DPI will not actuate, and if it does, it will 
not do it in the correct way. A substantial pressure drop is required for correct delivery which can only be achieved with a significantly deep breath. DPIs are not usually prescribed to patients under six, or for the very old.

Typically a DPI will deliver between $10-40 \%$ of the dose to the bronchi [5]. Problems arise when patients exhale into the actuator, as the added humidity can cause the delivery tube to clog with powdered medication, meaning that the efficiency will decrease and the ease at which medication can be inhaled will also be decreased.

\subsubsection{Pressurised Metered Dose Inhalers}

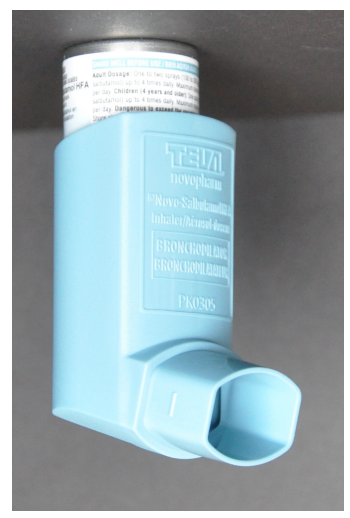

Figure 2.3: TEVA Novopharm salbutamol sulphate pMDI

pMDIs were first introduced in the Riker laboratories in the 1950s and since then have become the most commonly used device for aerosol medication delivery. Although the pMDI has developed to improve portability and convenience, the main aspects of the design remain unchanged since 1956, apart from changes due to the "Montreal Protocol" in 1987.

The pMDI itself has three main parts; the canister, the actuator and the metering valve. The canister holds the medication in solution or in particulate suspensions, 
along with a propellant (generally HFA-134a). The propellant mixes with the medication when the canister is shaken, and upon actuation the propellant causes the medication to spray out. The propellant must deliver an equal amount of pressure (meaning that a variable pressure gas cannot be used) throughout delivery, regardless of how full the canister is. For this reason, gases such as carbon dioxide cannot be used, as a nearly empty canister using $\mathrm{CO}_{2}$ would not generate enough pressure in actuation to deliver the medication with a high enough velocity. When pMDIs were first introduced, the propellant used would typically be a chlorofluorocarbon but since 1987, pMDIs have gradually evolved to use HFAs as they have similar thermodynamic properties to CFCs, but have less of a negative impact on the environment. This change in propellant resulted in the requirement for design changes to the actuator.

The canister is required to be able to withstand the high pressure of the propellant, and for this reason, glass and aluminium are the most suitable materials to use. Aluminium is used most commonly as it is less expensive than the glass alternative.

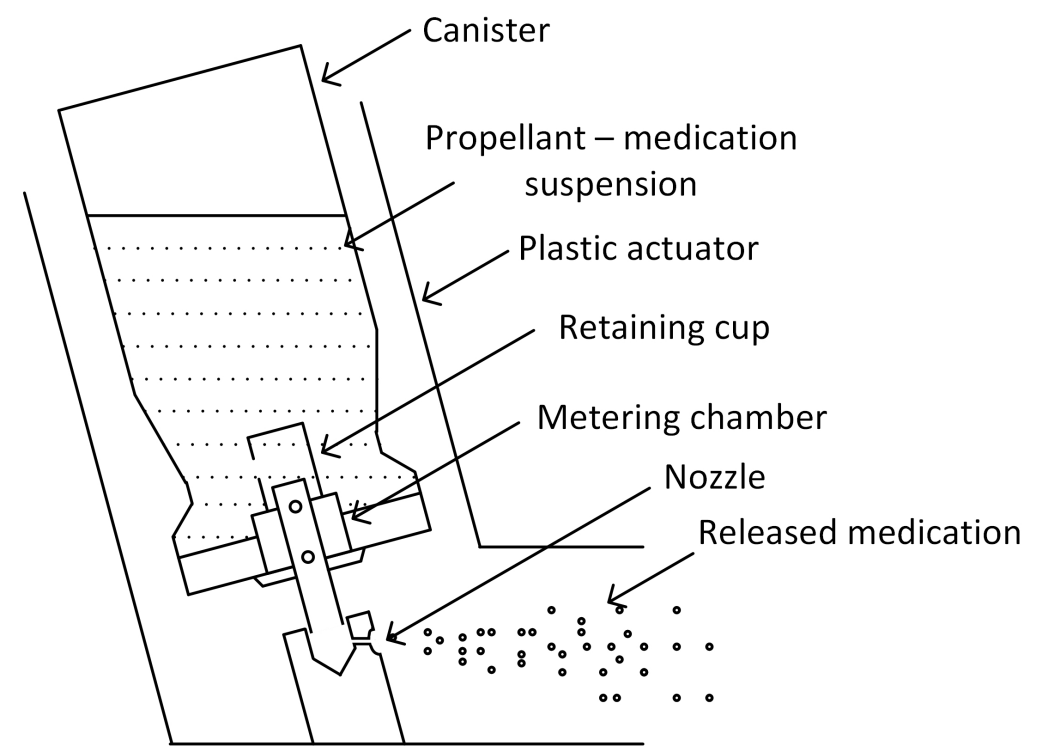

Figure 2.4: Diagram of actuator 
The canister fits directly inside the actuator; a plastic case which is held by the patient. The actuator has a built-in metering valve, which the nozzle of the canister fits into. Upon actuation, the patient pushes the canister into the actuator, and hence pushes the nozzle into the metering valve which then releases between 100 and $200 \mu \mathrm{g}$ of medication, which then passes through a small orifice. The size of this orifice dictates the size of the released particles, which in turn affects how much of the medication will target the correct area of the lungs (as discussed in Section 1.5.2). The angle of the spray is also affected by the size of this orifice, which is important to the efficiency of the inhaler, as the angle will effect drug deposition prior to reaching the lungs. A downwards angle of medication spray emitted from the actuator will result in higher deposition within the mouth and oropharyngeal tract, reducing the amount of medication that will reach the bronchi.

Because of the boiling point of the propellant and the pressure drop when it is released from the canister, the propellant will evaporate almost instantly upon actuation. This leaves only the medication to be inhaled, travelling at around $80 \mathrm{~m} / \mathrm{s}$ $[32]$.

\subsubsection{Propellants}

For a pMDI to work as they are supposed to, it is necessary to mix the medication with a propellant that will force the medication out of the actuator, and provide it with enough momentum to reach the back of the patients throat so that it can be inhaled into the lungs. Before 1987 dichlorodifluoromethane (CFC-12) was the primary propellant used for pMDIs. Different combinations of propellant and medication have been used with HFAs have been being chosen as the alternative. Possibilities for development of new propellants have also been suggested, though the changes in the actuating device that would be required makes the transition difficult. 
Propellants deliver constant pressure throughout the usage of the puffer and need to be non-toxic, as they are inhaled. Bioequivalents are difficult to produce because of these requirements, as well as additional factors such as melting point.

With HFA propellants, consistent dosing is provided regardless of how full the canister is, as has been found in Crosland [32], Cripps et al. [33], ROSS and GABRIO [34].

\section{Chlorofluorocarbons}

Dichlorodifluoromethane fulfilled the requirements of propellant in that it was nontoxic, in gas phase above $-24^{\circ}$ and (with the use of other added CFCs in small amounts) regulated vapour pressure. CFCs have a lower vapour pressure than HFAs and therefore the CFC canisters emitted doses at a lower velocity than the HFA canisters that are now used.

\section{Hydrofluoroalkenes}

HFAs were introduced in aerosol medication to replace CFCs. HFAs 227 and 134a are the most commonly used, but modifications have been required for the design of the actuator. For the propellant to combine correctly with the medication it is required that it is in liquid form whilst inside the canister, resulting in the HFA canisters being under high pressure. HFA actuators have a different metering valve and nozzle sizes to CFCs and therefore produce smaller particles, result in a higher proportion of respirable particles being released.

\subsubsection{Limitations}

pMDIs are not entirely efficient and have features which make them difficult to use for some patients, with the result that as little as $10 \%$ of the medication released in 
one dose will reach the lungs when using a pMDI alone [35]. Improper training in use of pMDIs from physicians is a major factor, as many patients do not know the proper procedure, but there are additional fundamental issues which impede the amount of medication that is available to the lungs.

When using a pMDI correctly, this high-velocity spray will deposit a large amount of medication within the mouth and oropharyngeal tract, meaning that rather than reaching the lungs it is swallowed instead. This is particularly important to note for corticosteroids as swallowing can cause side effects such as oral thrush (which is why patients are recommended to rinse their mouths after use). The high velocity spray also has a partially downwards angle which adds to the impaction of particles within the mouth $[2,3,32]$.

Another potential problem of using a pMDI is the "Cold Freon" effect, which results from the low boiling point of the propellant. HFA-134a and other propellants cool the medication during evaporation to as low as $-17^{\circ} \mathrm{C}$ [36]. When the cold plume hits the back of the patient's throat, it can have a freezing effect, resulting in the patient not inhaling properly. This effect is most commonly experienced by younger patients who are not familiar with pMDIs. Younger patients (as well as geriatric patients and patients with COPD) also find the correct use of a pMDI difficult due to the requirement to synchronise actuation and inhalation. Without immediate inhalation, all of the medication will deposit within the mouth resulting in ineffective treatment. This action is difficult for patients who do not understand the requirements and patients who have difficulty in controlling their breath. In patients with arthritis, the movement required for actuation can be difficult or impossible, in which case using a nebuliser or DPI would be more appropriate.

Because of these limitations pMDIs alone are not recommended for patients under six years of age and geriatric patients and it is recommended that all asthmatics own 
a spacer device for use in an asthma attack, when breath control is difficult.

\subsubsection{Nozzle diameter}

With the change in propellant, new nozzle diameters have been introduced in order to match the efficacy of a HFA propelled pMDI to that of a CFC propelled pMDI (which used $0.5 \mathrm{~mm}$ as a standard nozzle diameter). HFA-134a-propelled pMDI commonly have nozzle diameters of $0.25 \mathrm{~mm}$, but other diameters are available.

Newman et al. [37] studied the effect of changing from CFC propellant to different formulations of HFA, as well as studying the effect of the nozzle diameter on where medication would deposit. In this study, feneterol was radio-labelled with the radionuclide technetium 99m. This method of detection has been used in other studies, including Usmani et al. [38] and Newman et al. [39]. By radio-labelling formulations it is possible to trace the deposition pattern as location of the drug, after inhalation, can be captured using a gamma ray camera. The radionuclide is dissolved in chloroform and added to a canister similar to one which is used for medication. The chloroform is then evaporated by air and the medication and propellent solution from a normal puffer is transferred into the canister at a temperature of $-60^{\circ} \mathrm{C}$, at which the solution exists in liquid form. Adding a valve to the canister makes it possible to use in an actuator, and after administering as a pMDI, inhalation of the medication can be tracked using a gamma camera. Newman et al. [37] examined the oropharynx and the whole lung, divided into peripheral, intermediate and central zones for deposition. For deposition outside of the body (medication left on the on the actuator and medication exhaled), UV spectrophotometry (discussed further in Section 3.2.3) was used to quantify the amount of medication on each part.

In vivo experiments were carried out with three different types of propellant, CFC12, HFA-134a and HFA-227. The results of these experiments can be seen in Figure 


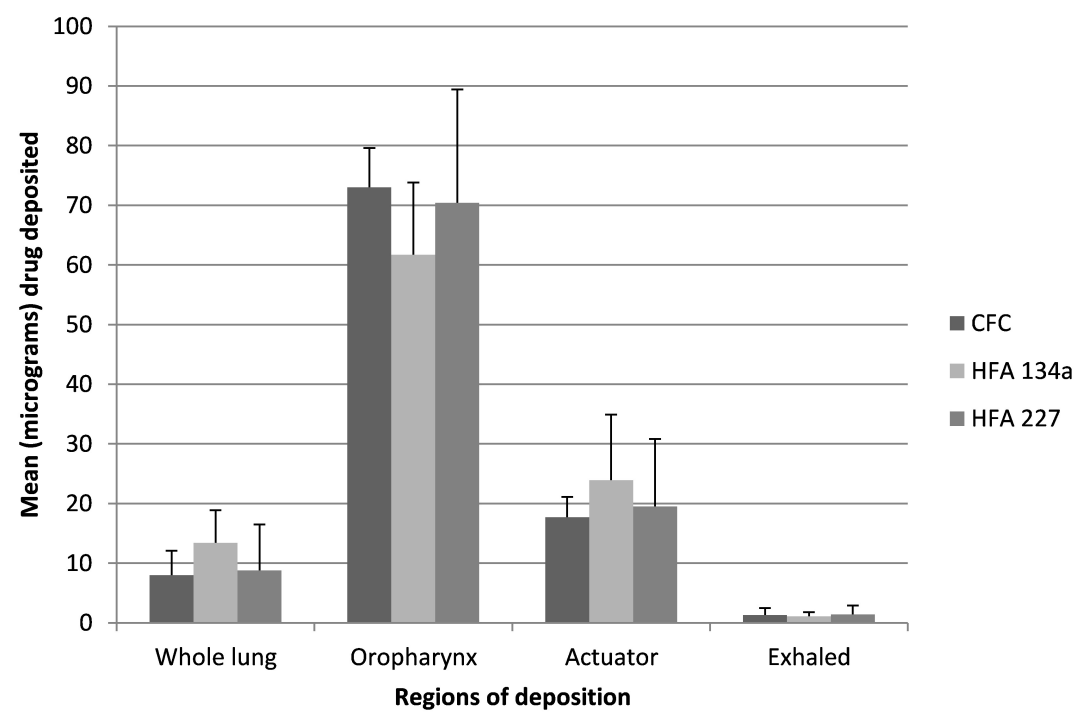

Figure 2.5: Effect of propellant on areas of deposition (adapted from Newman et al. $[37])$

From this it can be seen that the propellant used in a pMDI has significant effect on the amount of medication that will reach the lungs. The results illustrate that HFA-134a will result in higher amounts of lung deposition of medication and lower amounts of oropharyngeal deposition, suggesting that the change in propellant enhances deposition to the lungs. The lower values of deposition for the CFC propellant could be due to not only the properties of the released plume, but also because the "Cold Freon" effect is more common in CFC-propelled pMDIs, causing a patient to stop inhaling during actuation.

For the second part of their study, a variety of nozzle diameters were tested. The nozzle diameter of the actuator effects the size of the droplets that are released to the patient, and the results of the testing reflected this. HFA-134a propellant was used with actuators with nozzle diameters of $0.2 \mathrm{~mm}, 0.25 \mathrm{~mm}$ and $0.3 \mathrm{~mm}$. These three nozzle diameters were compared with a CFC actuator nozzle to study which HFA 
nozzle diameter would produce similar bioavailability of medication to previously used actuators. The size of the particles produced has an effect on the regions of the lung that can be targeted, important to the treatment of bronchospasm and bronchoconstriction. Smaller particles also have lower probability of impacting on the oropharyngeal tract, effecting the severity of side effects. The results of this study are shown in Figure 2.6, demonstrating that the the amount of medication that will reach the lungs is inversely proportional to the nozzle diameter. The results also showed that a nozzle diameter of $0.25 \mathrm{~mm}$ for HFA-134a would produce the most similar pattern of deposition to CFC propellant and nozzle diameter, which is what is desired for consistency in the treatment of asthma.

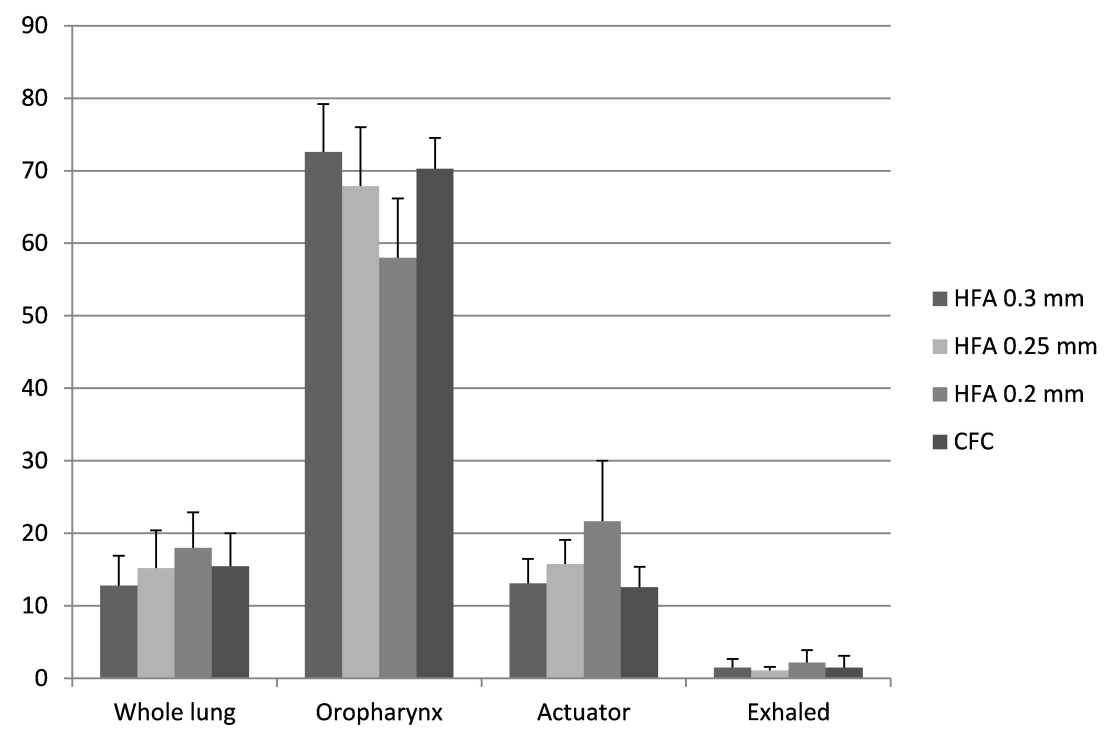

Figure 2.6: Effect of nozzle diameter on area at which medication will deposit (adapted from Newman et al. [37])

These results were also demonstrated when Kleinstreuer et al. [40] performed numerical studies with different propellants and actuator orifice diameters. From the results in Table 2.2 it can be seen that, once again, a smaller diameter of nozzle will result in a larger amount of medication reaching the lungs, due to the effect that 
nozzle size has on the MMAD of the released medication. These results also show that HFA propellants produce finer particles, due to the propellant surface tension and boiling point discussed in Section 2.4.4.

\begin{tabular}{lcc}
\hline & Nozzle diameter $(\mathrm{mm})$ & $\begin{array}{c}\text { Deposition efficiency in } \\
\text { the lung regions }\end{array}$ \\
\hline \hline CFC pMDI & 0.5 & $5.2 \%$ \\
HFA pMDI & 0.25 & $46.6 \%$ \\
CFC pMDI & 0.25 & $23.2 \%$ \\
\hline
\end{tabular}

Table 2.2: Comparison of different spray conditions (adapted from Kleinstreuer et al. [40])

\subsubsection{Spray properties}

Properties of the spray released from the pMDI influence the regions of the respiratory system (or add-on device) in which undesired deposition may occur. The study of spray properties enhances the ability to determine whether drug deposition occurs due to the turbulence of released flow or to the way in which the spray acts, which could cause inertial impaction on the walls of the spacer. It also gives insight into the way that released particles break up, their size distribution and the causation of this break up.

Dunbar [41] and Dunbar and Miller [42] detail studies of the velocity and droplet size of a pMDI using phase-Doppler particle analysis (PDPA) for the main spray and laser strobe micro-cinematography at the region close to the nozzle, in order to study the atomization process of the pMDI. Optical methods were chosen for their non-intrusive nature. Using a 3D traverse system, the 1D PDPA system obtained results for spatial analysis. These studies found that the pMDI spray is unsteady, 
transient, three dimensional and multiphase. Readings were taken at $25 \mathrm{~mm}$ intervals, measuring drop velocity, turbulence intensity and mean diameter of particles. These studies showed that the radial profiles of the spray were asymmetric, a phenomena that was studied further by Crosland [32].

The results of the temporal analysis of Dunbar and Miller [42] and Crosland [32] are shown in Figure 2.7. In these results the coordinates represent the $\mathrm{x}$ and $\mathrm{y}$ positions of the laser from the nozzle of the actuator. Similar to that shown by Kleinstreuer et al. [40], it was found that the CFC propellant would produce larger volume mean diameters than HFA propellant. It was noticed that for both propellants the released spray is pre-atomized, as no liquid ligaments were found. The analysis also showed that the spray cone angle fluctuated during the spray, which could influence oropharyngeal deposition, or deposition within an add-on device

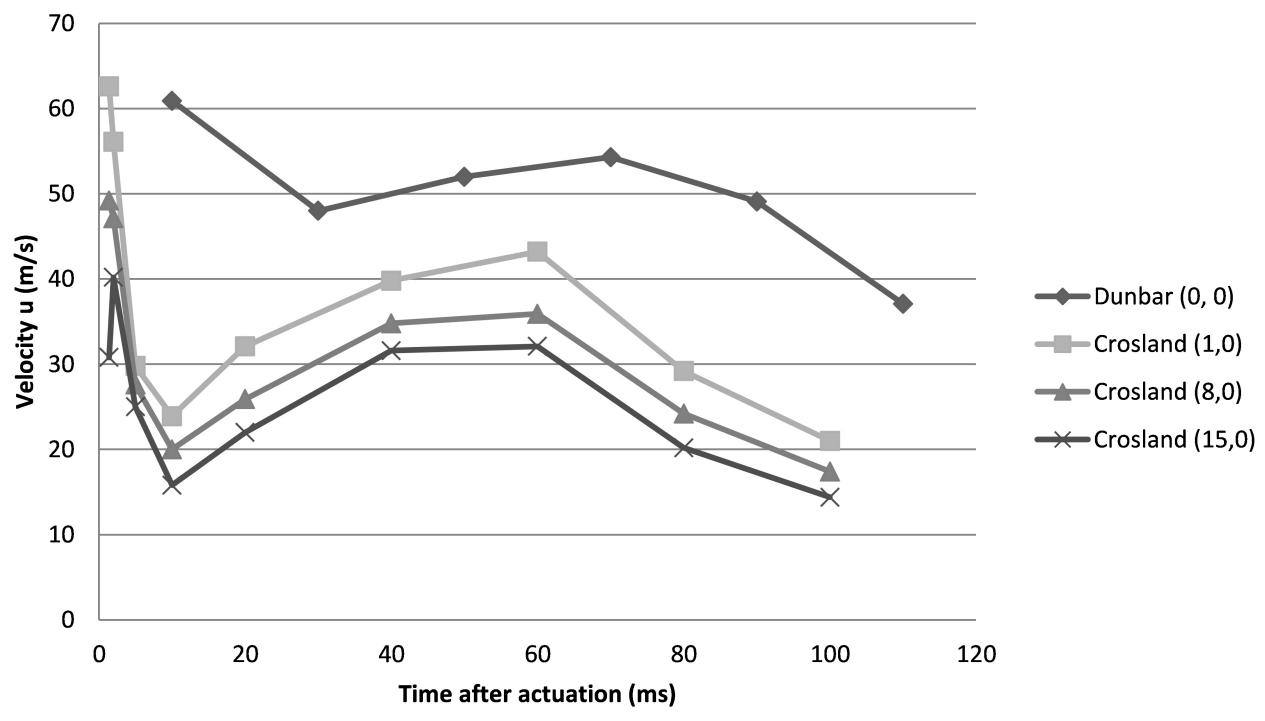

Figure 2.7: Temporal evolution of the velocity of the spray emitted from a Ventolinbrand pMDI (adapted from Crosland [32])

In Crosland [32], the spray characteristics were studied using particle image velocimetry (PIV) rather than PDPA. PIV spreads a sheet of laser instead of a single 
beam and from this, flow can be captured in an plane rather than a point. Using this technique it was possible to spatially resolve the velocity measurements of released spray. Two brands of HFA-134a propelled canisters were studied, establishing that the same flow characteristics were shown by both, implying that similar actuators therefore, with the same formulation of medication and propellant, can be expected to display similar spray characteristics. Actuators were also tested between lot numbers and between full and empty canisters, and no significant differences were found. A secondary air flow was also added in order to simulate a patient inhaling the medication at the same time as the medication being released. Measurements were carried out using a pMDI alone as well as using a spacer device, and it was found that with the spacer did not impact the spray velocity as the velocity measurements were similar to those without.

The study found that, as in Dunbar [41], the spray released from an actuator was asymmetrical. The spray cone angle and the direction of spray were both measured by considering the edge of the cone to be where the velocity was at half of its peak. The study found that spray direction from the pMDI varied with time, with the spray pointing downwards for part of the actuation, up to a maximum deflection of $5.5^{\circ}$. This deflection occurs in 30 milliseconds, and by $60 \mathrm{~ms}$ the spray returned to a horizontal position. It was at this point that the peaks in velocity would flatten for the remainder of the actuation, displayed earlier in Figure 2.7. The following relationships were produced as a result of the study, so that the horizontal and vertical components of flow could be found for a pMDI:

$$
u=a+\frac{b\left(\frac{y-c+d(f-2)}{d}\left(\frac{f}{2}-1\right)\right) \exp \left(-\frac{y-x+d(f-2)}{2 d}\right)}{(f-2)^{\left(\frac{f}{2}-1\right)} \exp \left(-\frac{f}{2}+1\right)}
$$




$$
v=\frac{a+c x+e x^{2}+g x^{3}+i x^{4}}{1+b x+d x^{2}+f x^{3}+h x^{4}}
$$

Where $a-h$ are curve fitting constants.

In Matida and Alhegagi [3], where spacer deposition was simulated, the results from Crosland [32] were used to find the spray angle emitted from an actuator, when related to time could be stated as:

$$
\alpha=\frac{1490.5}{t^{1.75}}\left[1-\exp \left(-0.000727 t^{2.5}\right)\right]
$$

\subsubsection{Particle distribution of aerosol sprays}

The distribution of particles within an aerosol spray impacts the regions in which the medication will deposit within the lungs, and will also impact the deposition in add-on devices. For this reason, distribution should be considered in numerical simulations of the spray from a puffer. The molecules in salbutamol sulphate are not mono-disperse due to the manner in which the particles are ejected from the puffer, and the different diameters are of importance in determining both how much medication will leave a spacer and where the medication will reach the lungs.

Particle size distribution in pMDIs was studied in Clark [43] where near-orifice analysis was performed using an aerodynamic particle sizer (APS), a system that uses a twin beam velocimeter to measure the time of particle release, which is related to the inertia and aerodynamic diameter of the released particles. Speculation exists around the behaviour of the spray when it exits the puffer; as flash evaporation has been suggested to cause break-up as well as shear at the outlet of the orifice. Clark [43], stated that the shear at the outlet of the orifice was the main impactor and that flash evaporation was less influential in particle break up. It was found that the 
diameter of particles could be found using:

$$
d_{i}=\frac{C_{5}}{q_{e}^{m}\left(\frac{P_{e}-P_{A}}{P_{A}}\right)} n
$$

Where $d_{i}$ is the initial droplet size, $q_{e}$ is the mass fraction in the vapour phase in the expansion chamber, $P_{e}$ is the pressure in the expansion chamber and $P_{A}$ is atmospheric pressure. $m, n$ and $C_{5}$ are constants, with the former two are dependent on droplet sizes, relating to 'quality of flow to droplet size' and 'pressure to droplet size', respectively.

The values for $C_{5}, m$ and $n$ were $8.02,0.56$ and 0.46 .

Dunbar and Miller [42] found that $q$ is a flow quality parameter for the mass mean diameter $\left(\mathrm{MMD}\right.$; or $\left.D_{0.5}\right)$ of the particle which is also used in the Rosin-Rammler distribution of particles. Rosin-Rammler distribution was introduced in 1933 by Rosin and Rammler [44], and was initially used to calculate the size distribution of coal but is adaptable for use with aerosols. The Rosin-Rammler distribution is a probability density function that states:

$$
p(d)_{m}=\frac{q d^{q-1}}{X^{q}} \exp ^{\frac{d^{q}}{}}
$$

Where $p(D)_{m}$ is the probability of a particle of size $D, q$ is the size distribution factor, and $X$ is the size constant.

The variation of the MMAD for salbutamol sulphate particles is also considered to be in Gaussian distribution, and vary depending on the device used with the medication. In Fink et al. [29], a tracheobronchial model was used alongside various delivery configurations, including using an HFA actuator with a spacer. Using a cascade impactor, the diameters of the particles were able to be measured, and plotted in order to determine the geometric standard deviation (GSD). With this configuration the 
MMAD of the particles was found to be $3.53 \mu \mathrm{m}$, with a GSD of 3.1.

The distribution found in Dunbar and Miller [42] has also been used in Oliveira et al. [45] in the simulation of flow. ANSYS packages include this function. The following parameters were used:

\begin{tabular}{cc}
\hline Parameter & Value \\
\hline \hline Minimum diameter & $1.22 \mu \mathrm{m}$ \\
Maximum diameter & $49.5 \mu \mathrm{m}$ \\
Mean diameter & $12.82 \mu \mathrm{m}$ \\
Spread parameter & 1.44 \\
\hline
\end{tabular}

Table 2.3: Parameters for Rosin Rammler (adapted from Oliveira et al. [45])

where spread parameter is the Rosin Rammler distribution of particles.

\subsection{Add-on Devices}

Add-on devices are components that attach to a pMDI in order to increase the distance between the actuator and the patient's throat, to reduce the requirement for breath-actuation synchronisation and to therefore increase the efficiency of the pMDI. Although most patients are able to use the pMDI alone, it is suggested that all patients prescribed a pMDI should be aware of how to use a spacer, as an acute asthma attack could reduce a patient's ability to synchronise actuation and inhalation. For children under six, it is essential that an add-on device is used; otherwise there is a $50 \%$ likelihood that the treatment will be inefficient.

As the distance between the throat and actuator is increased by using an add-on device, the "Cold Freon" effect decreases, as with an increased distance the propellant will have an increased amount of time in which to evaporate and for the temperature 
of the gas to rise. With valved add-on devices, the requirement for actuation and inhalation is reduced, as the medication plume will remain within the add-on device for a short period of time in which the patient can inhale. Also, with a distance within an add-on device, the initial high velocity spray has time to decrease, which will also decrease deposition occurring within the mouth, as less impaction will occur.

\subsubsection{Open-Tube Spacers}

Open tube spacers are the smallest and simplest add-on devices available to use with a pMDI. The principle of the open tube spacer is to increase the distance between the patient's throat and the dispensing nozzle so that the amount of time for the propellant plume to evaporate is increased, leaving smaller particles for inhalation, as well as increasing the amount of time for the particle velocity to decrease. Both of these will result in a decrease in medication deposited in the mouth and throat. It is still necessary for the patient to be able to breathe in as the pMDI is actuated when using open-tube spacers, as the device will not hold any of the medication.

\subsubsection{Reverse Flow Devices}

In reverse flow add-on devices, the medication plume is initially directed away from the patient into a bag or chamber with air vents. This type of spacer provides an alternative method of increasing the amount of time between actuation and inhalation, and allows the propellant to partially evaporate before reaching the oropharyngeal cavity, much like an open-tube spacer. 


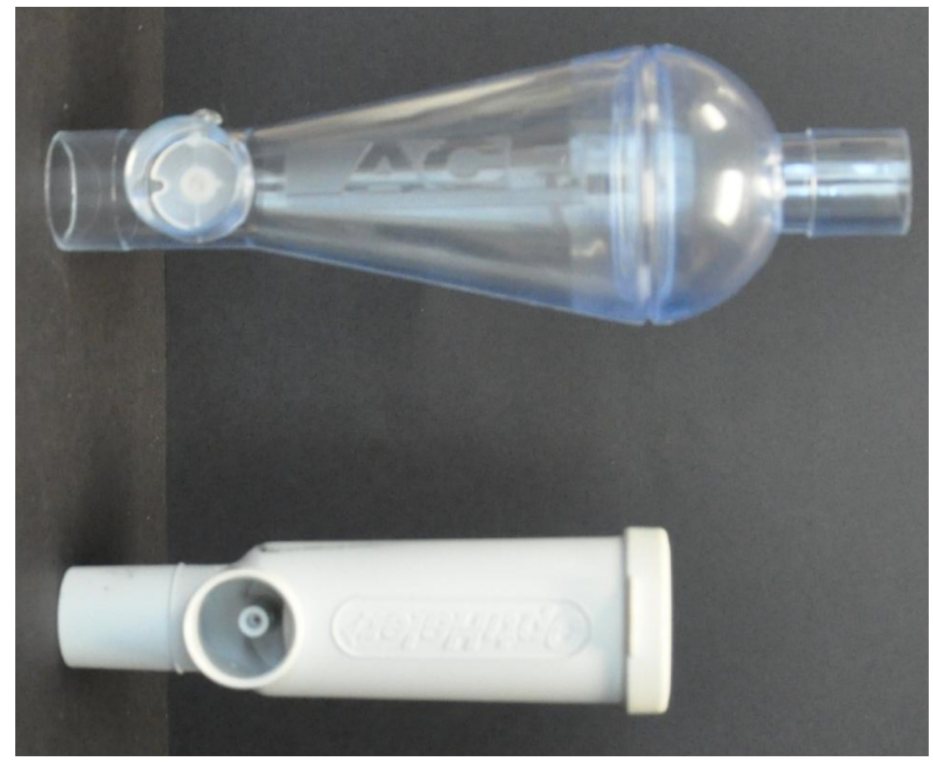

Figure 2.8: Reverse flow spacers

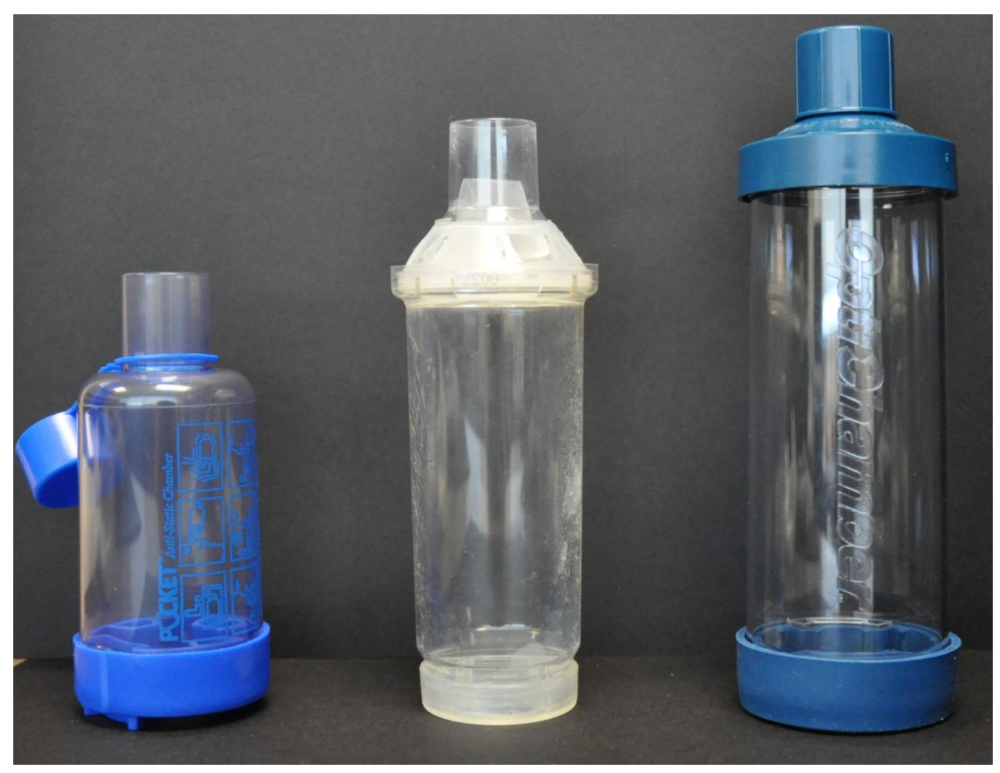

Figure 2.9: Different volume holding chambers 


\subsubsection{Holding Chambers}

Holding chambers are valved devices that attach to the pMDI. Medication is sprayed into a chamber and is then inhaled by the patient. As there is a valve, it is not necessary for the breath and actuation to be synchronised, though the medication filled air must be inhaled within a short amount of time. Holding chambers generally provide the largest distance between the actuator and the patient which is important for the velocity of particles, as the initial plume of medication will travel at approximately 80 $\mathrm{m} / \mathrm{s}$, which rapidly decreases to just $10 \mathrm{~m} / \mathrm{s}$ in $10 \mathrm{~cm}$. The decreased speed will have a beneficial effect on impaction on the oropharyngeal tract, and the time will allow the propellant to evaporate, leaving smaller, more efficacious, medicine particles. The volume of the holding chamber can allow the delivery of large volumes of medication in cases where it is required, such as in severe COPD.

There are a number of valved chambers that are available for use with a pMDI which vary in both volume and design. Straight spacers are available as well as pearshaped and cone-shaped spacers, in which the plastic design expands out from the mouth in a similar shape to the aerosol plume. Spacers are also available in a number of volumes of up to 1 litre. Volumes larger than this would not be appropriate as the breathing capacity of the patient would not exceed this, and therefore medication would be wasted as it would remain in the spacer.

Although it is desirable for large particles to condense on the walls of the spacer, leaving only the smaller particles to reach the respiratory tract, unwanted deposition can occur due to a number of factors. Because the spacers are not perfectly designed and the cone angle of the actuator varies, some useful particles will automatically impinge on the walls. 


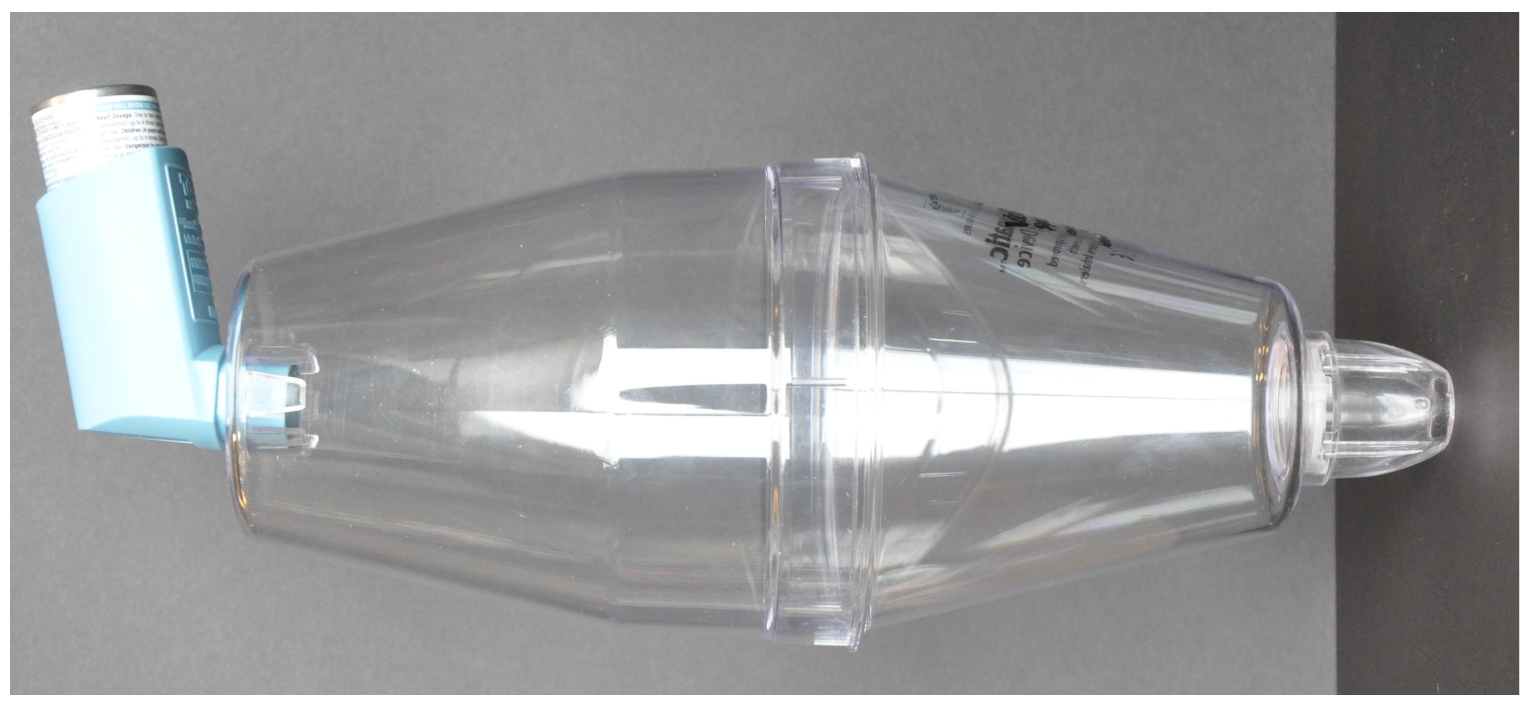

Figure 2.10: Allen and Hanbury's Large Volume Spacer

\section{Static Effects}

Typically, spacers are made of plastic, and electrostatic charges may build up inside the walls of the holding chamber. When this occurs the medication becomes attracted to the walls rather than passing through to the patient, and increased deposition within the device occurs. To minimise this, it is important that correct cleaning methods (rinsing with dish detergent and allowing to air dry) are used, and the spacer can be primed prior to use by coating the walls with a small amount of salbutamol by actuating a small amount of medication from the pMDI. Also available, though more expensive, are anti-static aluminium spacers, though the efficiency of these spacers has not been shown to be significantly higher than plastic spacers used with the correct washing procedure.

\subsection{Add-on device studies}

Add-on devices come in a variety of volumes and shapes and assist in treating patients who have difficulty in using a pMDI alone, as well as in some cases increasing the 
efficacious dose of medication for a patient. Studies have been carried out to determine the importance of shape and size, and how the use of a spacer can improve the delivery of medication from a pMDI.

Bisgaard et al. [12], Barry and O'Callaghan [7] and Newman [36] considered generically shaped spacers with different dimensions. In these studies the length and diameter of the spacer were varied in order to further evaluate the optimal shape for add-on devices. Bisgaard et al. [12] studied the effects of the spacer length on the size of particles that reach the patient. It is preferable for the spacer to emit a larger portion of fine particles (between 2 and $5 \mu \mathrm{m}$ in diameter) to the patient, because of the region of the lungs that these particles will target. In the study, budesonide was emitted from a pMDI into metal spacers of various lengths. Impaction of larger particles resulted in medication being lost within the spacer, and longer spacers allowed more time for particles to break up into the "fine" range of particle sizes. These results can be seen in Figure 2.11. The results also showed that the ratio of the coarse and fine particles varied with length of the spacer, and from this is can be seen that while a longer spacer released more fine particles, a spacer of length $13 \mathrm{~cm}$ released the optimal ratio of coarse to fine particles.

As well as the length of the spacer, studies show that the diameter also has an effect on the volume and on deposition. In Newman [36] and Barry and O'Callaghan [7] the diameter of spacers, as well as length, was varied. The results (shown in Figure 2.12) showed that with sodium cromoglycate particles there was a significant correlation between increased spacer diameter and the amount of medication recovered. It also can be seen that with increasing length the amount of sodium cromoglycate recovered increased, complimenting the findings of Bisgaard et al. [12] and also suggesting that increased volumes increased the number of recovered particles. 


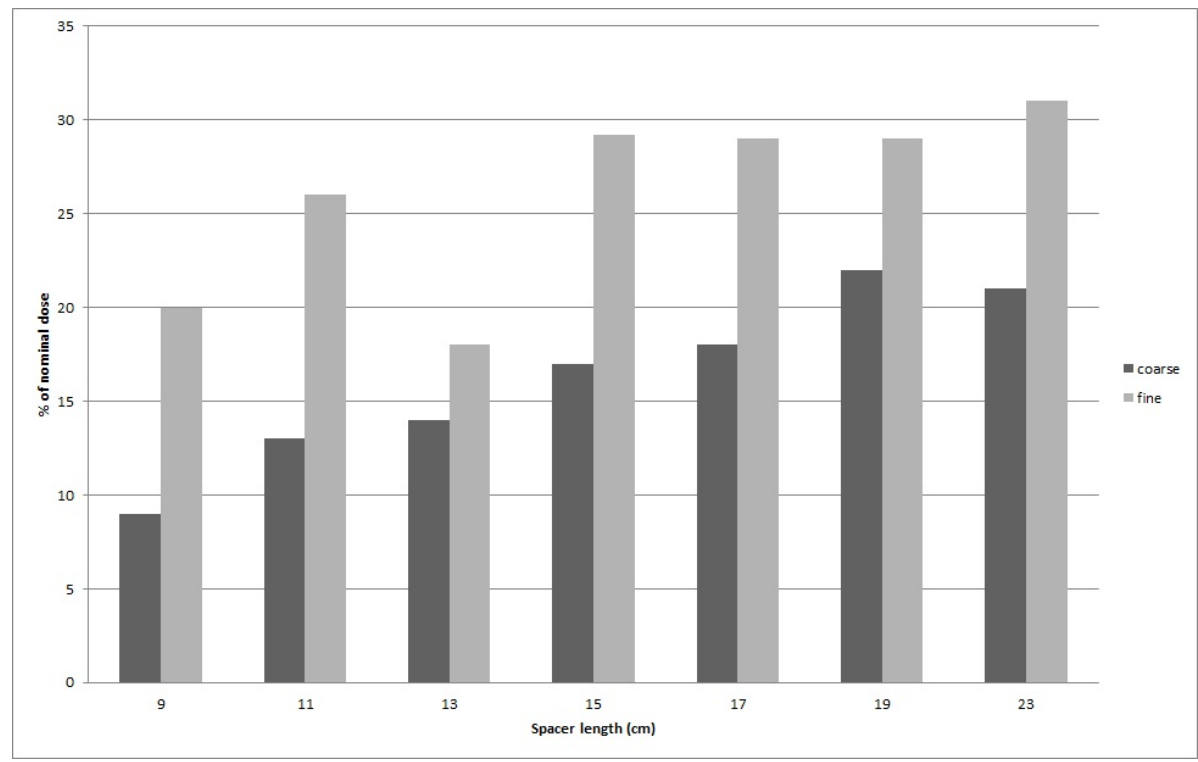

Figure 2.11: Effect of spacer length on the particle sizes that will reach patient (adapted from Bisgaard et al. [12])

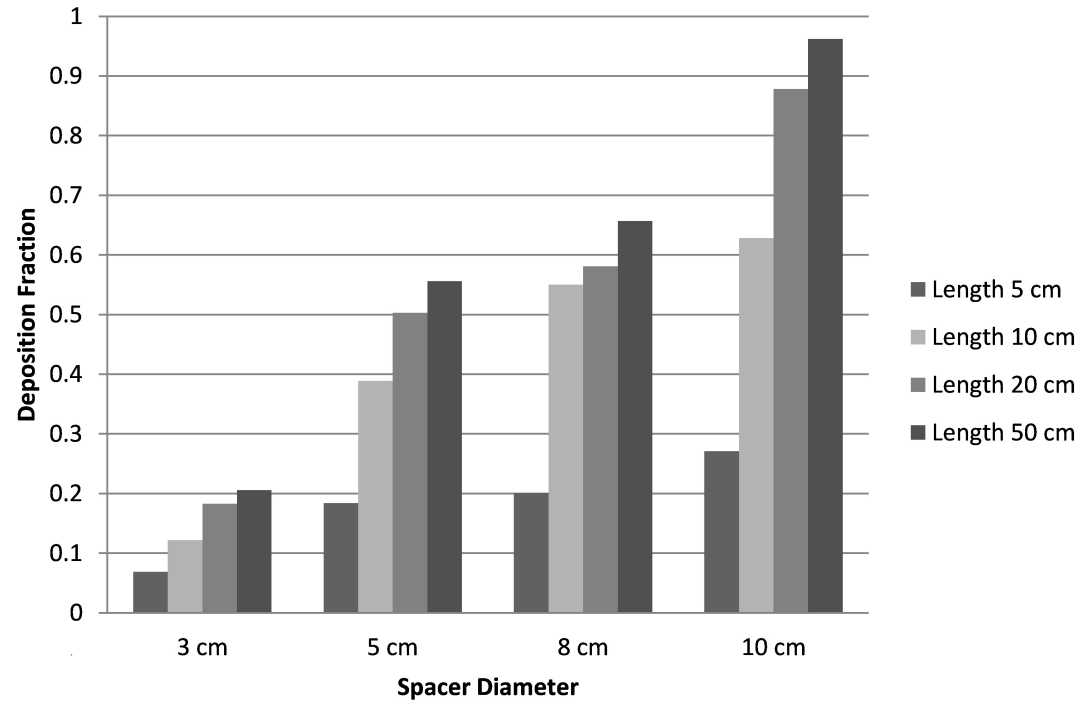

Figure 2.12: Effect of spacer diameter on the particle sizes that will reach patient (adapted from Newman [36]) 
Barry and O'Callaghan [7] went on to study the effect of volume of spacers. As can be seen in Figure 2.13, the amount of medication delivered to a patient increases up to a volume of around $1000 \mathrm{ml}$. After this point the benefits are much less prominent, a finding echoed in Newman [36], which states that for spacers larger than $1000 \mathrm{ml}$ in volume it is difficult for a patient to inhale the entire contents in one breath. Larger volume spacers that require more than one breath to inhale have been found to be less beneficial as, in the time taken to inhale a second time, a greater amount of respirable particles may deposit on the spacer walls. It is recommended that spacers should not be significantly greater than the maximum inspiratory flow capacity for this reason.

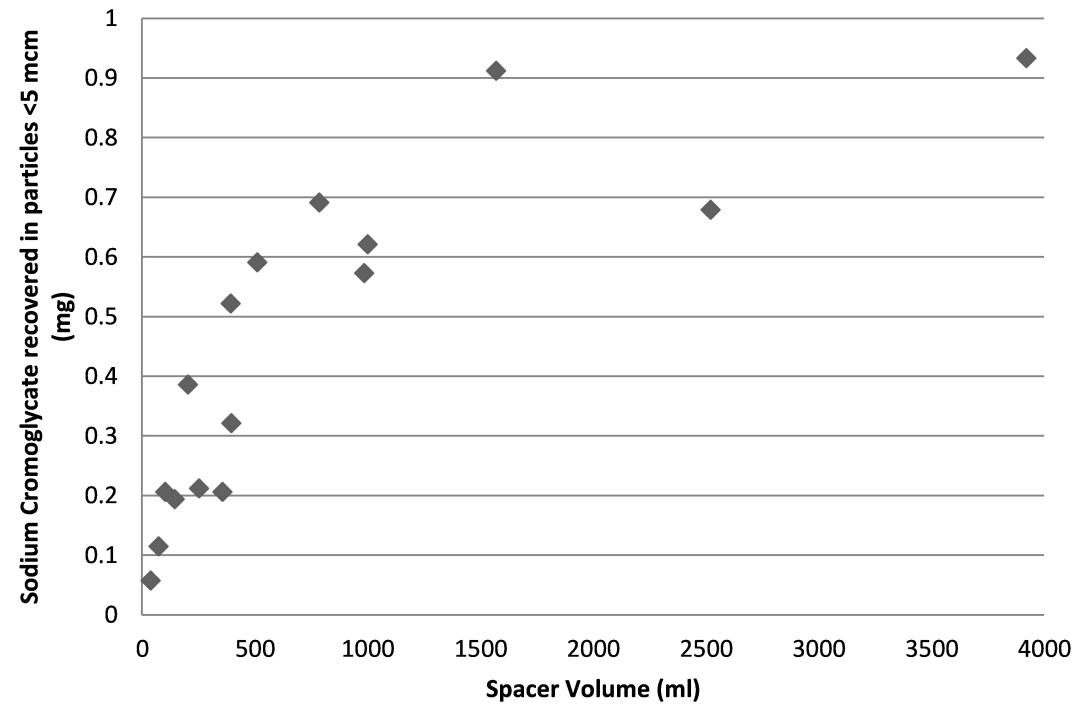

Figure 2.13: Effect of spacer volume on the amount of particles less than $5 \mathrm{mcm}$ (adapted from Barry and O'Callaghan [7])

Barry and O'Callaghan [6] studied the drug delivery from different available spacer devices. In their research they considered different volumes of spacers as well as different shapes that are available as treatment options to assess the effect of design on medication delivery. The characteristics of four of the seven tested spacers are as 
follows:

\begin{tabular}{cccc}
\hline Spacer & Shape & Length $(\mathrm{cm})$ & Volume $(\mathrm{ml})$ \\
\hline \hline Volumatic & Diamond & 23 & 700 \\
Inspirease & Cylindrical & 11.3 & 650 \\
Aerochamber & Cylindrical & 11 & 145 \\
Dynahaler & Cylindrical & 12 & 60 \\
\hline
\end{tabular}

Table 2.4: Characteristics of spacer devices tested (adapted from Barry and O'Callaghan [6])

In vitro studies were performed using a glass multi-stage liquid impinger (MSLI), a device which can attach to a spacer or puffer and to a compressor in order to simulate different flow rates. An MSLI contains levels at which different particle sizes will be collected, in order to determine the composition of the flow in terms of particle diameters, and MSLIs are used frequently in determining the MMAD of particles from devices. Salbutamol, budesonide and sodium cromogycate were used in this study. In order to measure the amount of salbutamol and budesonide deposited, the MSLI and filter were washed with ethanol and methanol respectively (due to the solubility of each drug in each solvent) and then high-pressure liquid chromatography was used. To measure sodium cromoglycate deposition, UV spectrophotometry was used after washing the MSLI levels and filter with water.

The study showed that large volume spacers increased the fine particle dose (particles smaller than $5 \mu \mathrm{m}$ in diameter) whereas smaller volume chambers would reduce the amount of fine particles that would reach the patient. In the study of sodium cromoglycate, it was found that the type of spacer was not significant, and the amount delivered to the patient was more dependent on the actual volume. It was found that the MMAD of all released particles from spacers would be smaller than when the pMDI was used alone, a trend which was present for all three medications. 
When spacers were tested using salbutamol it was found that the Volumatic ${ }^{\mathrm{TM}}$ spacer increased the fine particle dose available for inhalation whereas the smaller volume spacers reduced the fine particle dose compared to using a pMDI. The results are shown in Figure 2.14.

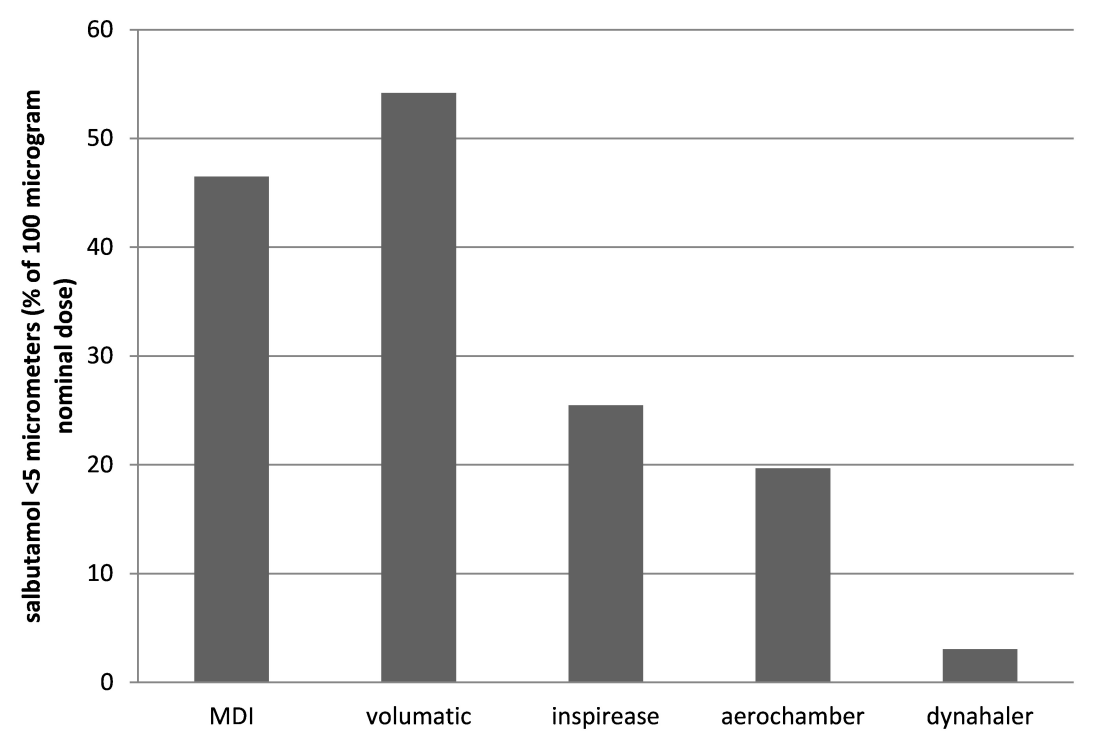

Figure 2.14: Salbutamol deposition for different spacers (adapted from Barry and O'Callaghan [6])

Finally, in the testing of budesonide it was found that the type of spacer used had little impact on the amount of medication that was released to the patient.

From this study it was concluded that while smaller holding chambers and spacer devices do not increase the aerosol drug delivery to the lungs, larger volume spacers will increase the number of particles released in the respirable range. For this reason, large volume spacers, especially when cone shaped, are more effective than using a pMDI alone. It was found, however, that if a pMDI is used correctly then the addition of a smaller volume spacer is not beneficial.

Barry and O'Callaghan [8] also detailed the behaviour of commercially available spacer devices, namely the Babyhaler ${ }^{\mathrm{TM}}$, Volumatic $^{\mathrm{TM}}$ and Aerochamber ${ }^{\mathrm{TM}}$. This 
research studied the the inspiratory flow rate effect on the medication dose that would reach a patient. An MSLI was used with flow rates of $20 \mathrm{~L} / \mathrm{min}$ and $60 \mathrm{~L} / \mathrm{min}$ for different medications. The results of this study are shown in Figure 2.15, and demonstrate that the differences in deposition in flows varied in the Babyhaler ${ }^{\mathrm{TM}}$ more than other devices, dependent on the drug-spacer combination. From this variation in results for each different medication, it can be deduced that spacer devices need testing for each medication that they will be used for, as results cannot be extrapolated.

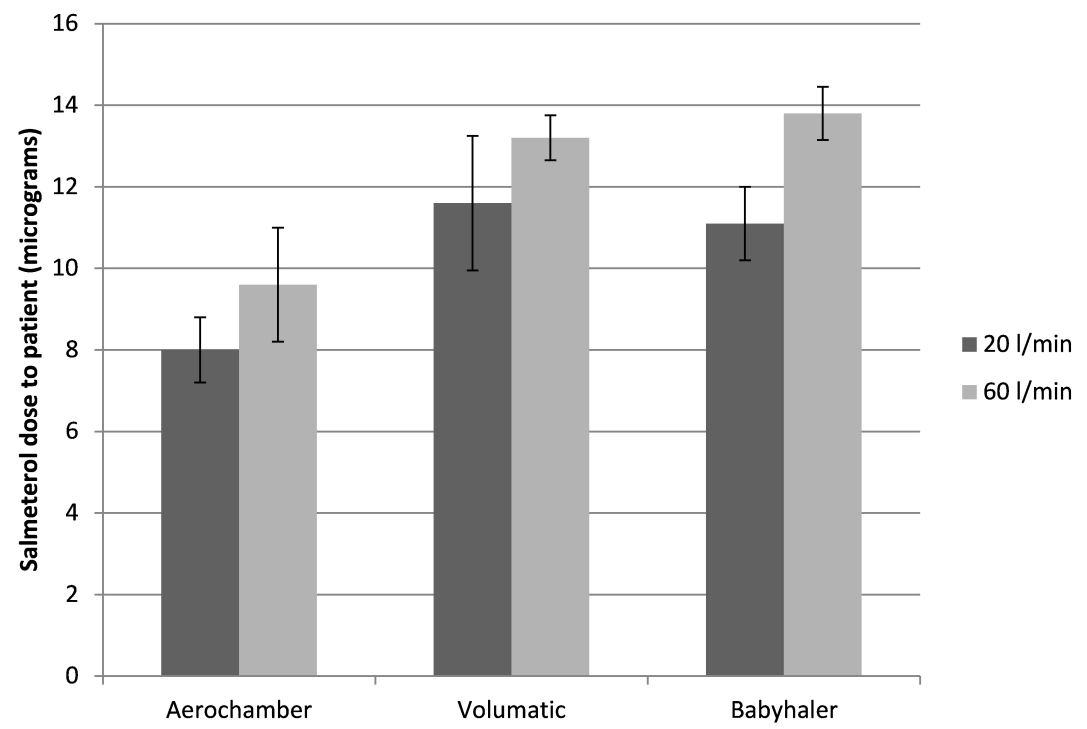

Figure 2.15: Salmeterol dose to patient using different commercially available spacers (adapted from Barry and O'Callaghan [8])

\subsection{Inspiratory flow rate}

The rate at which a patient inhales is also of interest when using devices for treatment in asthma, because of the impact that it has on the regions of the lung that the medication will reach. Although pMDIs do not have a specified inspiratory flow rate (which DPIs do), the flow at which a patient inhales will impact the location of lung 
targeting as discussed in Section 1.5.2, and will also have an effect when a patient is using a spacer.

Newman et al. [39] compared the use of a pMDI alone to the use of a pMDI with a spacer at fast and slow inhalation rates. Sodium cromoglycate was used along with a radio-tracer that allowed the deposition of medication to be monitored. Technetium as pertechnetate was used to radio-label the sodium cromoglycate. This meant that once inside the body the medication could be traced using gamma scintigraphy, and the medication remaining within the spacer was quantified using UV spectrophotometry (rinsing the parts in distilled water and testing at a wavelength of $326 \mathrm{~nm}$ ). To monitor deposition within the body, a gamma ray counter was used, taking readings at different areas of interest. The flow rate at which the patient breathed was monitored using respiratory inductance plethysmography (measuring the movement of the chest wall).

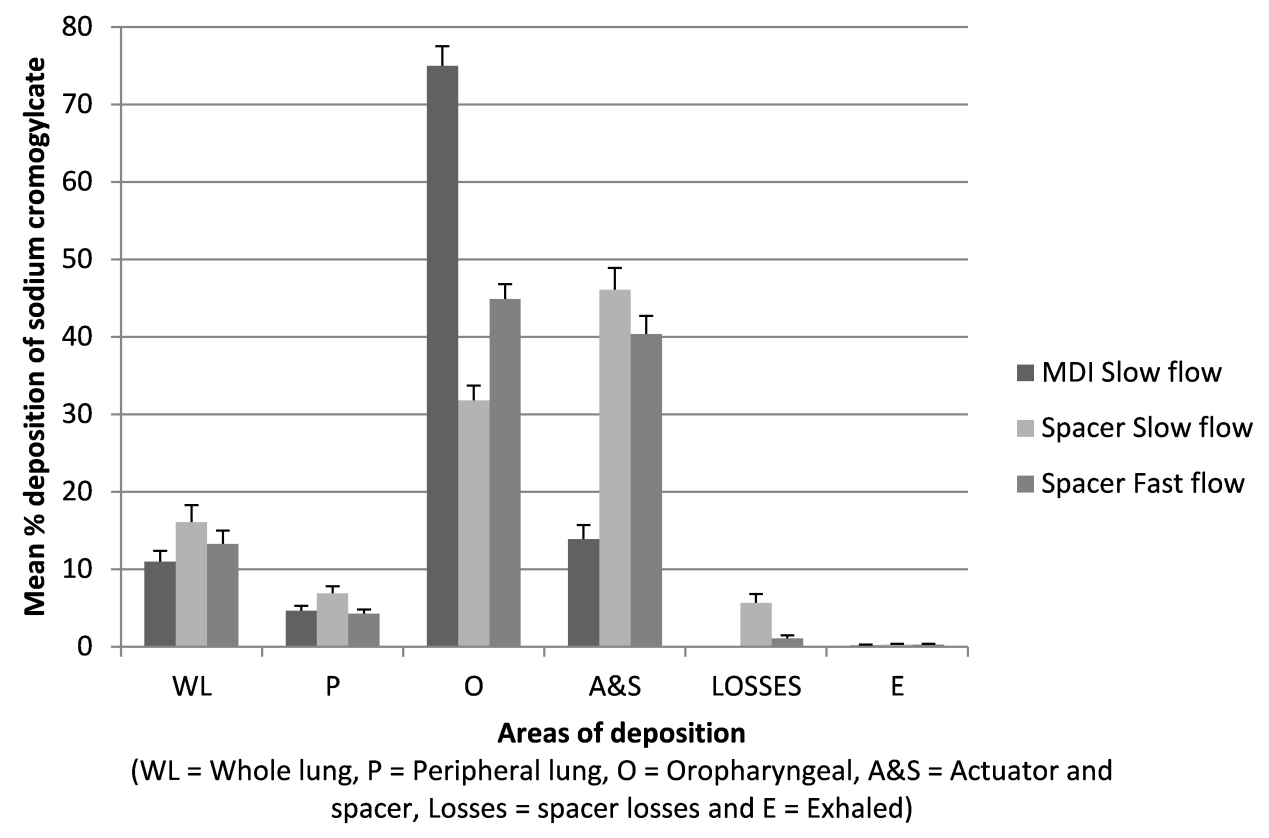

Figure 2.16: Areas of deposition with fast and slow inhalation rates (adapted from Newman et al. [39]) 
From Figure 2.16 it can be seen that for a pMDI used with a low inspiratory flow rate, a high amount of oropharyngeal deposition will occur, which is echoed in most available literature $[38,39]$. It was also found that slow inspiratory flow rates with a spacer would result in the lowest amount of oropharyngeal deposition, but the study does not detail the areas of deposition within the lungs, and as a result it is not possible to determine whether this will enhance the efficacy.

Usmani et al. [38] also studied the effect of inhalation flow rate on particle deposition in different parts of the lungs, studying the regional delivery of albuterol. Slow inspiratory flows were considered to be between 30 and $60 \mathrm{~L} / \mathrm{min}$ and fast flow rate to be higher than $60 \mathrm{~L} / \mathrm{min}$. Mono-disperse albuterol particles were generated using a spinning disk in order to assess which particles would penetrate the different parts of the respiratory tract. Radio-labelling was used with gamma scintography to detect where the particles deposited, and 2D planar imaging was used to analyse where deposition occurred.

The research found that inspiratory flow was important when considering the efficiency of aerosol deposition within the respiratory tract for albuterol, and that the size of the particles also determined the sites at which medication would deposit. Smaller particles had higher lung deposition, although it was considered that these smaller particles would penetrate too deeply into the lungs to aid bronchoconstriction. The results showed that for all particle sizes, central lung deposition would increase with a higher inspiratory flow rate. It was also shown that faster inspiratory flow rates were only beneficial for smaller particles, and would cause significant decrease in lung deposition for large particles, suggesting that at $60 \mathrm{~L} / \mathrm{min}$ a greater number of particles would impinge on the orophyrangeal tract, which is undesirable due to the side effects that occur as a result of this.

Large-volume spacers, in particular the Volumatic ${ }^{\mathrm{TM}}$ spacer, have been the topic 
of numerous studies performed by Oliveira et al. [45-47]. In these studies a numerical approach is taken, whereby the flow within the spacer is simulated using computational fluid dynamics. From these studies, new designs have been developed based on particle tracking of simulated medication, in such a way that the efficiency of the spacer was improved by modifying the shape. The valve of the spacer has also been considered in these studies. As with other available literature, these studies show that spacers will reduce the oropharyngeal deposition that occurs with pMDIs alone, and therefore side effects are also reduced when they are used. The MMAD of particles that reach a patient is also lower, meaning that they are more likely to reach correct areas of the lower respiratory system, and show that the larger particles are deposited within the spacer device itself rather than in the upper airways.

Alhegagi and Matida [2] studied the drug delivery from a number of spacers, with results showing that larger volume spacers delivered more medication to the patient. In this thesis and in Matida and Alhegagi [3], the non uniformity of medication distribution is also considered, with a spacer being divided into upper and lower halves for analysis. From both experimental and numerical results it was possible to see that a greater amount of deposition occurred on the lower half of the spacer, agreeing with the findings of Crosland [32], that the released spray from the pMDI fluctuates downwards during flow, and that this would influence the deposition from a pMDI. Turbulence models were tested in the numerical part of the study, the results of which suggested that the deposition was mainly due to the inertial impaction rather than turbulence occurring in the spacer.

This thesis aims to fill the gaps in the literature by considering the deposition within a large volume spacer. The spacer will be considered in four quadrants in order to analyse the regions in which medication is depositing, to verify results found by Alhegagi and Matida [2] and to further study the asymmetry in medication deposition 
within a spacer. The effect of inspiratory flow rate is also considered, with inhalation rates of as low as $7.5 \mathrm{~L} / \mathrm{min}$ and as high as $60 \mathrm{~L} / \mathrm{min}$ being tested.

Pressure measurements are also taken during physical experimentation in order to apply a more realistic representation in numerical analysis of spacer deposition, and to study the effect that this will have on the flow. Previous work has included particle diameter distribution (Oliveira et al. [45]) and the spray angle of the spacer (Alhegagi and Matida [2]) but has not incorporated the two methods. Currently, only steady state simulations exist for the use of a pMDI with a spacer, despite the spray emitted from a pMDI being described as transient in Dunbar and Miller [42] and Crosland [32]. This thesis aims to develop a transient simulation within a large volume spacer, and to quantify the amount of medication deposited within the spacer using particle tracking. The purpose of this is to assess the flow rate that is beneficial for use with a large volume spacer, and to assess the effect of the proposed downward angle in flow found by Crosland [32]. Analysis will be performed experimentally and using ANSYS CFX. 


\section{Chapter 3}

\section{Methodology}

Physical experiments were performed using the Volumatic ${ }^{\mathrm{TM}}$ spacer, a large volume spacer that is used in the treatment of asthma especially for younger patients. This spacer was chosen as previous research performed by has reported upper and lower medication deposition in medium sized spacers, and overall deposition in small and medium sized spacers, but did not include results for spacers with a volume of over $500 \mathrm{ml}[2,3]$. The Volumatic ${ }^{\mathrm{TM}}$ spacer is a diamond shaped spacer with a volume of $750 \mathrm{ml}$ and is a commonly prescribed spacer for the treatment of asthma in children. In this chapter the set up of the physical experiment is described. This description includes details of the equipment itself and how it was used in testing the different parts of the spacer at different rates of inspiratory flow.

\subsection{Experimental Description}

Experiments were performed in order to determine characteristics of the flow within a Volumatic $^{\mathrm{TM}}$ spacer when used with a Novo-Salbutamol HFA (Teva Pharmaceutical Industries ( ) pMDI. As discussed in Section 2.2, salbutamol sulphate is a commonly 
prescribed beta $_{2}$-agonist medication for the relief from acute asthma. The NovoSalbutamol HFA is also compatible with the Volumative ${ }^{\mathrm{TM}}$ spacer, a factor important in using puffers with spacers for patients. The main objectives of the experiment were to establish the quantity of salbutamol sulphate that remains within the spacer after actuation, the areas where the deposited medication is most concentrated and the main cause of deposition. From these findings, it is possible to deduce the efficiency of the spacer (in terms of the amount of medication that remains within the spacer, and hence how much would reach the patient) at different inhalation rates, and it will enable more realistic numerical simulations of the flow that is released from the actuator. Previous studies show that the cone angle from the nozzle causes downward flow, which leaves a higher deposition of medication on the lower portion of the spacer $[2,32]$. Experiments also show the effects of different inspiratory flow rates in order to study the extent to which the inspiratory flow rate affects deposition.

For testing, the method described by Alhegagi and Matida [2] (and repeated in Fiset [4]) was utilised whereby a vacuum pump was used to simulate different inspiratory flow rates. Five flow rates between $7.5 \mathrm{~L} / \mathrm{min}$ and $60 \mathrm{~L} / \mathrm{min}$ were used in order to replicate low to high breathing intakes. The equipment used was a Welch ( D Dry Vacuum Pump 2585B, a Brooks @ Mass Flow Meter 5863S, an Omega @ Pressure Transducer PX409, two National Instruments ${ }^{\mathrm{TM}}$ Data Acquisition USB-6009 devices and an Agilent 8543 UV-Visible Spectroscopy system. The data acquisition devices and Agilent Spectrophotometry systems were connected to a computer with Agilent and Labview ${ }^{\mathrm{TM}}$ software installed to enable instrument readings to be taken. 


\subsection{Equipment}

The following section lists the equipment and materials that were used in carrying out the experiments.

\subsubsection{Flow Meter}

The flow meter used was the Brooks Mass Flow Meter 5863, which uses a thermal mass flow sensor to determine the flow rate. The temperature change that occurs as a gas flows over a heated $\left(8^{\circ} \mathrm{C}\right)$ sensor tube is measured, and this temperature change is converted to an electrical signal which is read by the National Instruments ${ }^{\text {TM }}$ Data Acquisition Device. The flow meter can read flows up to 1000 litres per minute, and the output electrical signal takes a value between 0-5 V dc. Under factory set calibration conditions, the flow accuracy is $\pm 0.7 \%$ of the flow rate. The flow meter was calibrated using factory calibration that was checked prior to use, using LabView in order to relate the voltage output to the flow rate. The Labview program then captured the flow rate for the duration of each of the experiments.

\subsubsection{Pressure Transducer}

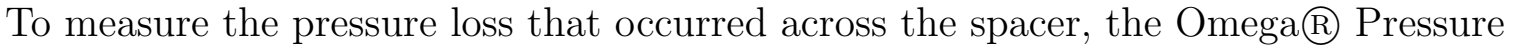
Transducer PX409 was used. The transducer was connected using an adapter to the outlet of the spacer in order to measure the pressure difference between the spacer and the atmosphere, and hence the pressure drop occurring within the spacer.

The Omega PX409 Pressure Transducer uses silicon wafer technology to measure pressure. As silicon is a piezoresistive material, its resistivity will vary with strain, which provides an electrical signal that can be read by data acquisition instruments. Piezoresistive pressure transducers comprise of a thin deformable diaphragm with 
four resistors placed in a Wheatstone bridge formation. When pressure is applied, the membrane will respond mechanically, and this response will be relayed as a voltage signal to the data acquisition device.

The transducer output a signal between $0-5 \mathrm{~V}$ dc. For pressure differences less than 1 psi (as were those in the experiment) the error difference is $\pm 0.5 \%$. Factory calibration was also required for the pressure transduced to relate the voltage to the pressure. Again using LabView, a program was written which would output the pressure readings from the experiments, which were analysed with a Fast Fourier Transform.

\subsubsection{UV Spectrophotometer}

The Agilent 8453 UV-Visible Spectrophotometer was used in measuring the absorption of each sample after the wash procedure. This system is a single beam spectrophotometer that uses light with wavelengths between 190 - $1100 \mathrm{~nm}$, meaning that it was suitable for use with salbutamol sulphate which shows peak absorbance between 276 and $280 \mathrm{~nm}$ [26]. The components of the UV-Visible spectrophotometer are shown in Figure 3.1. Two lamps are used in order to achieve the range of wavelengths; a deuterium arc lamp for the UV range (between 190 - $800 \mathrm{~nm}$ ) and a tungsten lamp (which will emit light with wavelength over the range of $370-1100$ $\mathrm{nm})$. These two sources of radiation are collimated using a lens that will minimise dispersion of the light, and this collimated light is passed through the sample when a reading is being taken (at which point the shutter will open to allow the light to be passed through). A stray light correction filter also ensures that negative effects of stray light for low wavelengths, below $190 \mathrm{~nm}$, which would not be required for the reading chosen. For readings taken of salbutamol sulphate during experimentation, an absorbance of $280 \mathrm{~nm}$ was used, meaning that the detector would compensate for 
radiation outside of the expected band.

After passing through the sample, the light is then refocused using a spectrograph lens before passing through to the detector. The slit that the light passes through is the exact size of one photo diode in the array, resulting in only the appropriate photo diode being projected onto by the band of wavelengths. This light is then dispersed by a concave holographic grating, at an angle proportional to the wavelength. The holographic grating consists of parallel grooves on a reflecting surface. The light that reflects from each mirror interacts with the light from the neighbouring groove. This interaction produces constructive interference if the waves are in phase, and out of phase waves will not propagate. The photo diode array that the light is dispersed onto is made up of 1024 photo diodes, which give the reading through a semiconductor chip of the intensity of the light.

\section{Theory of Spectrophotometry}

The absorbance of a solution is the amount of incident light that is absorbed. The spectrum is determined by the material and its composition, which in this case is the salbutamol sulphate. The absorbance for salbutamol sulphate was read at 280 $\mathrm{nm}$ as a fixed wavelength to study the measured sample data. The peak is used as the change in the absorbtion is greatest at this point, giving greater sensitivity and accuracy in the readings that are taken. It also results in a decreased impact from impurities in the sample. The intensity of the sample will vary multifunctionally with the wavelength, and hence the results are used to calculate the concentration. From this, and the knowledge of the volume of solvent used for analysis, the total deposition can be calculated. Beer-Lambert law is used to create the calibration curve between the absorbance and the concentration of salbutamol sulphate. 


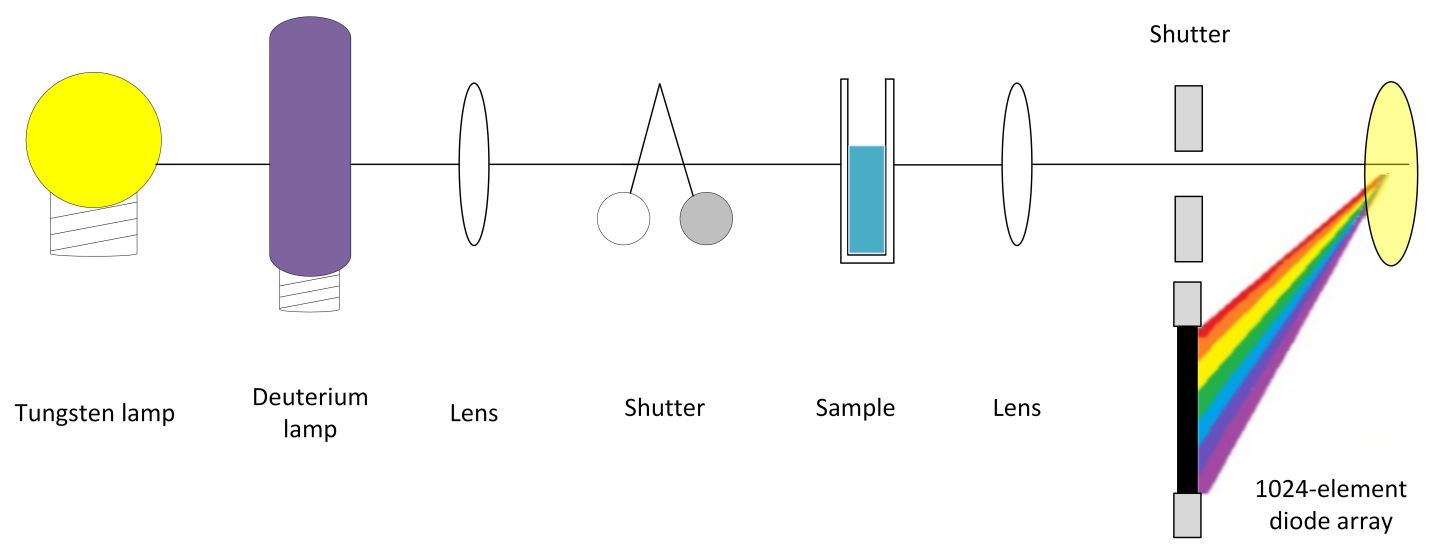

Figure 3.1: How UV works

\section{Beer-Lambert Law}

The calculation of the concentration of salbutamol sulphate present in each sample is calculated using a combination of two laws; Beer's law and Lambert's law.

Lambert's law, also known as Bouger's law, states that the transmittance of a sample is the percentage of the photons of light transmitted that will pass through the cell. Lambert's law was introduced in 1760 , but is stated to have been first discovered by Bouger in 1729, resulting in the relation having two names. The definition can be stated in the following way:

$$
\tau=\frac{I}{I_{0}}=e^{\epsilon b}
$$

Where;

$\tau$ is the transmittance

$I$ is the transmitted intensity

$I_{0}$ is the incident intensity

$\epsilon$ is the extinction or absorption coefficient of the solvent

$b$ is the path length (in cm for experiments)

The extinction coefficient is not only dependent on the substance but is a characteristic that is dependent on the wavelength, solvent and temperature of the sample. 
It is also dependent on the characteristics of the individual instrument and for this reason a predefined coefficient cannot be offered for a substance, but instead a calibration curve must be created.

Beer's law is similar to Lambert's, but considers the concentration of the solution rather than the intensity of absorbtion. The law states that the amount of light absorbed by the sample will be proportional to the number of absorbing molecules through which the light passes. Combining the two laws gives the following equations:

$$
A=-\log _{10} \tau=-\log _{10} \frac{I}{I_{0}}=e^{\epsilon b c}
$$

Where;

$A$ is the absorbance of the substance, $c$ is the concentration of the substance.

Using these equations the absorbance can be related to the concentration of the substance using the following equation

$$
c=k_{1} A
$$

Which can be rearranged to give:

$$
k_{1}=\frac{c}{A}
$$

Where $k_{1}$ is the calibration coefficient.

By testing known concentrations of salbutamol sulphate and calculating the calibration coefficient it was possible to curve fit the results of the carried out experiments in order to calculate the concentration in each sample. 


\subsubsection{Salbutamol Sulphate}

For experimentation, Novo-Salbutamol HFA (Teva Pharmaceutical Industries $®$ ) was used. Novo-salbutamol is a brand of salbutamol sulphate which, as described in Section 2.2, is a beta ${ }_{2}$-agonist which is used for treating acute asthma. Salbutamol sulphate is the preferred drug for the treatment of acute bronchospasm and is prescribed to the majority of asthmatics, as described in Section 2.2 .

\subsubsection{0\% Ethanol Solution}

Anhydrous ethanol and distilled water were used together to act as a solvent for the salbutamol sulphate. The anhydrous ethanol was diluted to a $20 \%$ solution, in accordance with the method described by Alhegagi and Matida [2]. Concentration selection was based on research performed by Ali et al. [48] which suggested that a lower concentration of ethanol enhances the solubility of salbutamol sulphate. According to Habib et al. [49], a concentration of $20 \%$ ethanol will allow the assay of salbutamol sulphate to be accurately determined. The evaporation rate of $20 \%$ ethanol solution at room temperature meant that in the time taken for each experiment, the concentration and volume of solvent would remain constant.

\subsection{Experimental Set up}

The equipment is arranged according to the schematic shown in Figure 3.2. A 3D

model of the Volumatic ${ }^{\mathrm{TM}}$ spacer (volume $750 \mathrm{ml}$ ) was produced using CREO ${ }^{\circledR}$ SolidWorks software and an ABS printer. This model was divided into four quadrants (displayed in Figure 3.3); upper and lower front sections and upper and lower back sections. An adapter was also modelled for attaching to the back sections in order to 


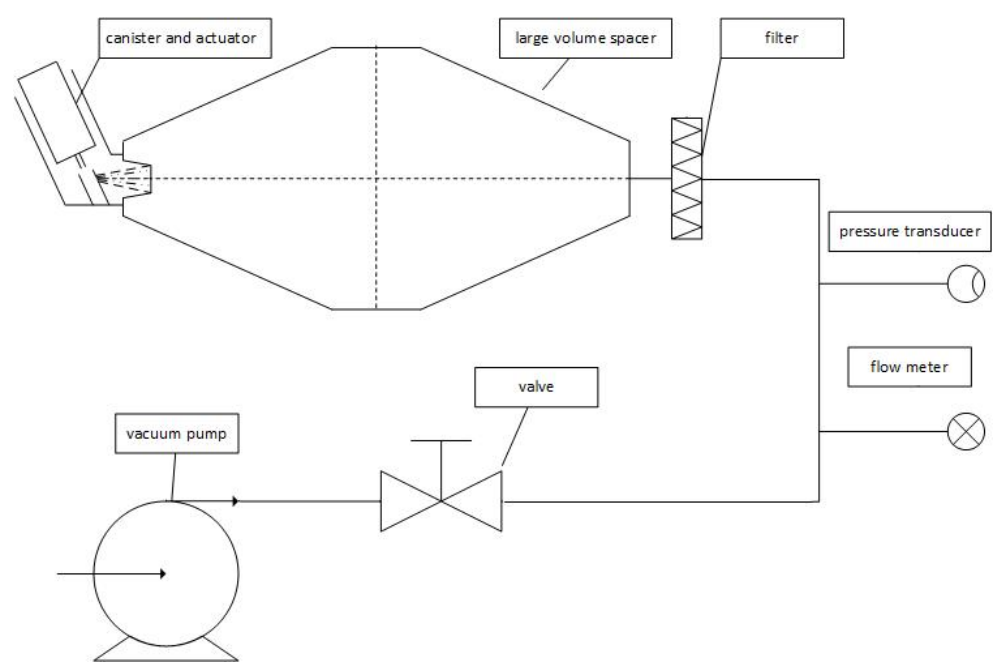

Figure 3.2: Schematic of experiment

fit the replica spacer to a Respirgard $\mathrm{II}^{\mathrm{TM}}$ filter. The filter was used to represent the

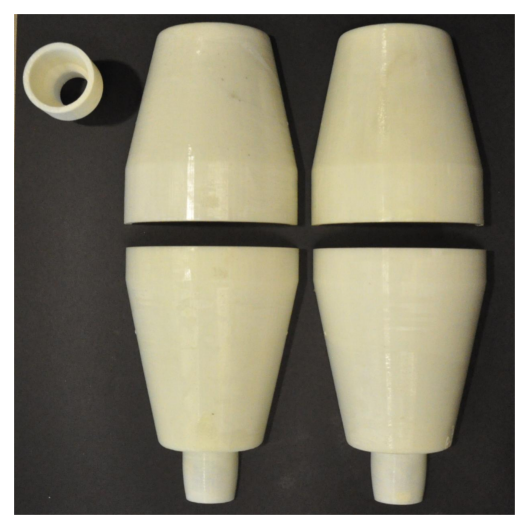

Figure 3.3: Spacer replica in four quadrants

geometry beyond the spacer (which would be the amount of medication that would reach the patient). The material of the filter is polypropene and salbutamol sulphate is recoverable from it after impaction, when the material is rinsed with ethanol solution. The four quadrants and adapter were attached together using dowel pins at fixed mid-positions and medical tape. A length of Parafilm ${ }^{\mathrm{TM}}$ was wrapped around the geometry and filter to prevent medication being released to the surroundings, or for inaccuracies in the flow through the set up. The filter was attached to a pressure 
transducer using medical tape and a short length of Parafilm ${ }^{\mathrm{TM}}$. In order to achieve consistent positioning of the spacer, and to enable the system to be set up in one plane, a wooden box was built with an inlay of Styrofoam ${ }^{\mathrm{TM}}$ fitted to the spacer. This set up is shown in Figure 3.4.

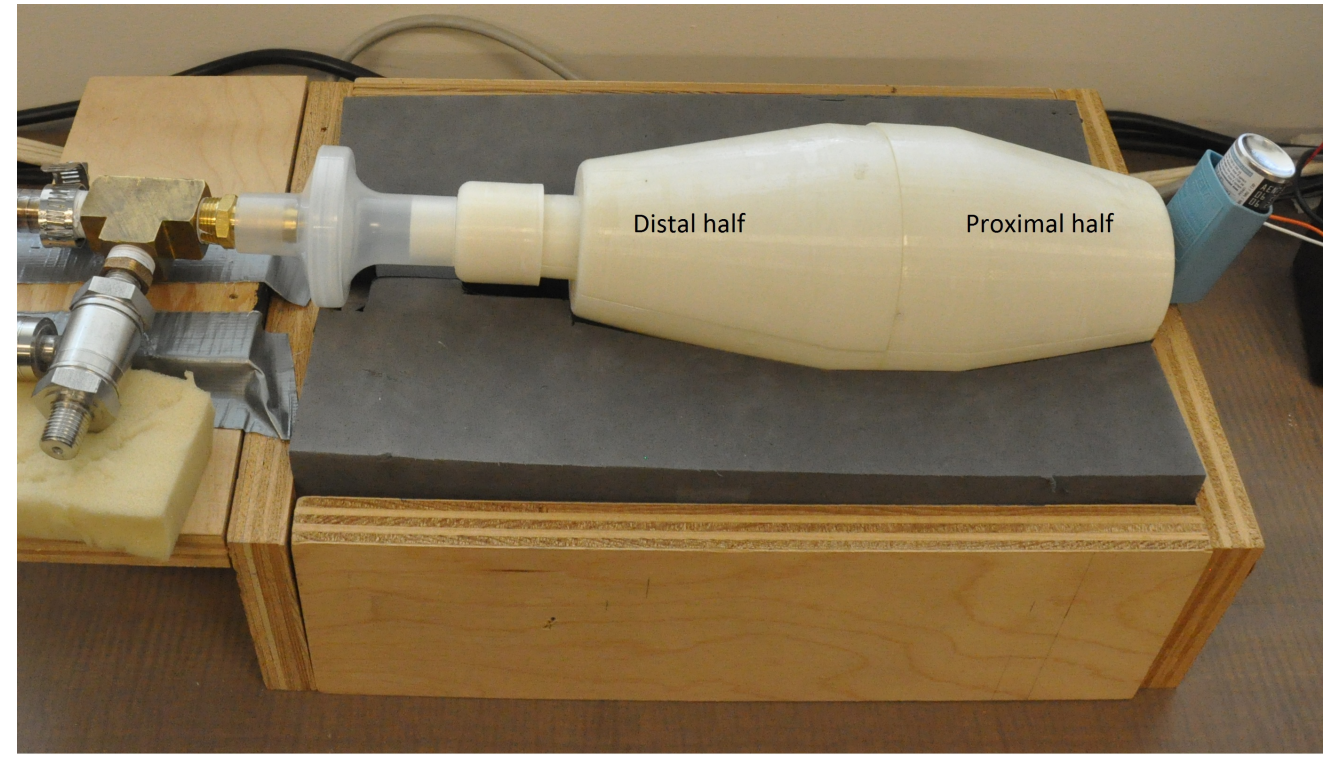

Figure 3.4: Wooden holder for spacer with pressure transducer attached

Using 1 foot of 0.5 inch inner diameter rubber tubing the pressure transducer was connected to an in-line Brooks@ Mass Flow Meter 5836S, also raised by a wooden box. Both transducer and flow meter were connected to a $\mathrm{PC}$ with Labview $^{\mathrm{TM}}$ installed, using National Instruments ${ }^{\mathrm{TM}}$ NI USB-6009 data acquisition devices.

Using another foot of tubing, the flow meter was connected to a valve (raised

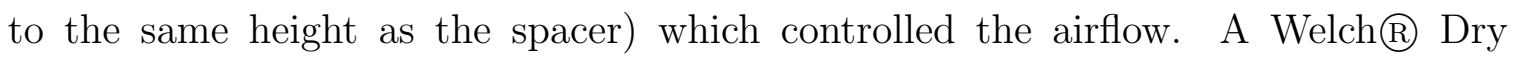
Vacuum Pump 2585B was connected in order to create the flow that represented a patient's inspiration which could be varied, using the valve, to achieve the desired flow rates. All equipment, apart from the vacuum pump, was positioned in-line to allow a full flow to develop (as shown by Figure 3.5) across a workbench. It was found that the vacuum pump caused vibrations strong enough to affect the readings 
of the data acquisition devices, and for this reason the pump was placed on the floor to avoid the frequency of the vacuum appearing in pressure readings; thus adding to experimental errors. Connections between instruments and tubing were secured using ring clamps and Teflon tubing tape to ensure that air was not lost, which would affect the measured flow rate.

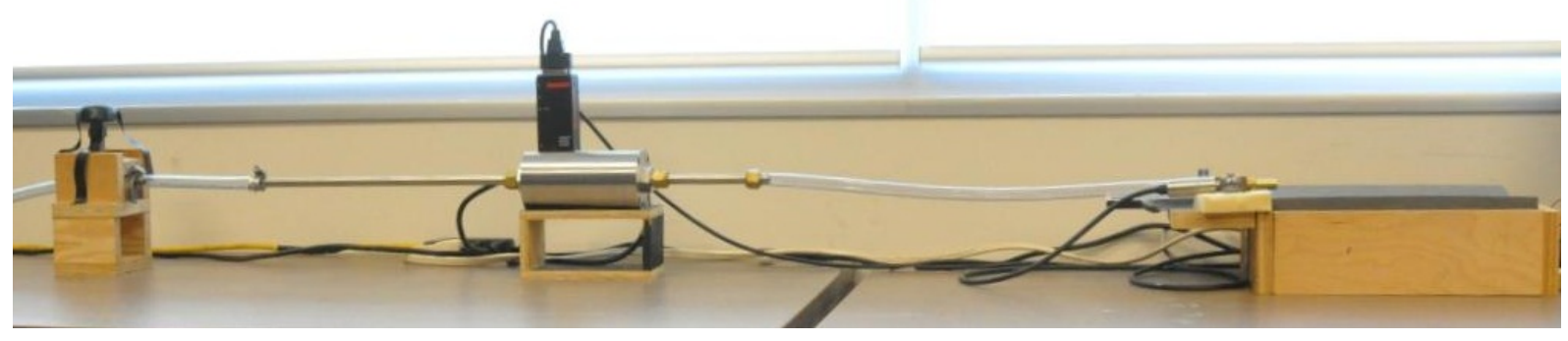

Figure 3.5: Experimental Set Up

\subsection{Experimental Procedure}

Prior to any experiments it was necessary to wash the Volumatic ${ }^{\mathrm{TM}}$ spacer with dish soap and allow to air dry. This action minimises the effects of static attracting the medication to the walls, which would cause an increased amount of deposition within the spacer, as discussed in Section 2.5.3. The equipment was then set up as described in Section 3.2. The flow rate first had to be set using the valve, flow meter and Labview ${ }^{\mathrm{TM}}$ program. Experiments were carried out at $7.5 \mathrm{~L} / \mathrm{min}, 15 \mathrm{~L} / \mathrm{min}, 30$ $\mathrm{L} / \mathrm{min}, 45 \mathrm{~L} / \mathrm{min}$ and $60 \mathrm{~L} / \mathrm{min}$, ranging from just above quiet breathing for a small child, to the inhalation flow rate for optimal gas exchange in most patients.

According to the manufacturer's instructions, the actuator and canister were shaken vertically 20 times before actuation, and a priming actuation was fired before delivering any medication into the system, to ensure that all actuations into the 
spacer would behave consistently. The pMDI was then fired 20 times into the spacer, being shaken vertically once between each actuation, to ensure consistency in the

dosage. After the final actuation the vacuum pump remained at the same flow rate for 30 seconds before stopping, to ensure that the desired flow rate was present for the entirety of the time that the medication plume would be in motion, and to ensure consistency was present for all experiments.

\subsection{Wash Procedure}

After running the experiment, the four quadrants of the spacer, the filter and the actuator were separated for analysis. Using $20 \mathrm{ml}$ of $20 \%$ ethanol solution, each part was washed separately in individual Petri dishes. Using a $10 \mathrm{ml}$ pipette to draw up the $20 \%$ ethanol solution from the parts available $20 \mathrm{ml}$ of solution, each part was initially rinsed 10 times before using a sterile cotton tipped applicator to clean the surface. Each part was then rinsed a further 10 times to ensure all medication was removed from the spacer and dissolved into the ethanol solution, again taking from the $20 \mathrm{ml}$ solution. $20 \mathrm{ml}$ was chosen as it would be a sufficient amount to take more than one sample for absorbance readings, and rinsing 20 times would ensure that the majority of medication was removed from the surface.

The filter (shown in Figure 3.6) and adapter were cleaned using the same solution, representing the medication that would be released from the spacer. The adaptor was cleaned using the same method as the actuator and the other parts of the spacer. To clean the filter, the $20 \mathrm{ml}$ of $20 \%$ ethanol solution was passed through the filter five times. Between each rinse through a pipette suction method was used, as in Alhegagi and Matida [2], in order to remove remaining liquid from the filter, so as to ensure that medication would not remain within the cotton. It was found that this was a 
sufficient number of times to fully rinse the filter, and further rinses had little impact on the amount of medication removed.

Quartz cuvettes were used to hold the solutions during measurements for UV absorbtion. Prior to use, each cuvette was rinsed twice using the solvent (20\% ethanol) and then once more using the sample that they were to be used for. Kimwipes $\mathbb{R}$ were used to clean the cuvettes between samples, and acetone was used to remove any remaining solution.

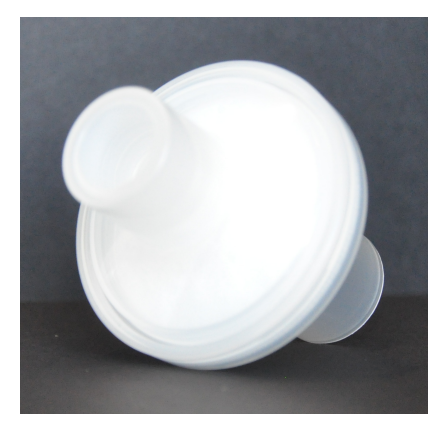

Figure 3.6: Respirgard $\mathrm{II}^{\mathrm{TM}}$ filter

\subsection{Calibration}

In order to determine the amount of salbutamol sulphate that deposited on each quadrant of the spacer it was necessary to generate a calibration curve between the measured absorbtion and the concentration of salbutamol sulphate, as described previously in Section 3.2.3. To achieve this curve, a pMDI puffer was actuated 100 times into a beaker, wide enough that the puffer could fit in entirely. Because of the behaviour of the medication, particles bouncing back from the beaker were not taken into consideration, as the medication would stick to the glass wall at the base of the beaker upon impaction. The salbutamol sulphate deposited within the spacer was then weighed using Sartorius scales. The salbutamol sulphate was then diluted with 
$100 \mathrm{ml}$ of $20 \%$ ethanol solution, and using the Agilent spectrophotometer and comparing against a control sample of $20 \%$ ethanol solution, the absorbtion was found. Further dilutions were made in order to find the absorbtion of salbutamol sulphate at enough points to create a calibration curve.

\subsection{Deposition Measurements}

In order to measure the amount of salbutamol sulphate deposited in each quadrant of the spacer, spectrophotometry was used to measure the absorbance of each sample taken during the wash procedure in Section 3.5. The spectrophotometry system is connected to a PC via CAN interface and the Agilent software for the spectrophotometer was used to document and visualise readings.

Salbutamol has a maximum absorption wavelength $(\lambda)$ of 276 - $280 \mathrm{~nm}$ (found in both the literature and in confirmed by experimentation of absorbance maxima and minima [2]) and a fixed wavelength mode at an absorbance of $280 \mathrm{~nm}$ was used for all readings. As it was used as a solvent in cleaning all parts, a blank of $20 \%$ ethanol solution was used as a control for all measurements. 


\section{Chapter 4}

\section{Numerical methods}

Using ANSYS CFX, simulations were performed using a replica of the Volumatic $^{\mathrm{TM}}$ spacer to study regions of deposition, aiming to create a realistic representation of the flow at different inhalation rates. ANSYS CFX was chosen due to previous work being carried out using this software, and its abilities in Lagrangian particle tracking and shear stress transport turbulence models. This chapter introduces both the geometry that was used for simulation and the theory that the software package ANSYS CFX uses to solve the unsteady Reynolds averaged Navier Stokes equations.

\subsection{Geometry}

The geometry of the combined spacer and puffer was created using ICEM CFD by ANSYS. The spacer was simplified at the outlet, with a straight rather than curved outlet to match that of the replica spacer mentioned in Section 3.3, but otherwise matched the dimensions of the Volumatic ${ }^{\mathrm{TM}}$ spacer, with a length of $200 \mathrm{~mm}$ (230 mm including valve section) and maximum diameter of $90 \mathrm{~mm}$. Four parts were created, as for the replica spacer, so that regional deposition could be studied. The puffer used was similar to that in Alhegagi and Matida [2] and Matida and Alhegagi 
[3], where the medication inlet modeled at the surface of the actuator nozzle with a diameter of $1.49 \mathrm{~mm}$, rather than at a point of $0.25 \mathrm{~mm}$. By projecting backwards from the surface of the actuator stem it was possible to create the required nozzle size in such a way that accurate meshing in this area was possible. An unstructured mesh was created from the geometry, and was created using tetrahedral elements as shown in Figure 4.1. Prism layers were used close to the walls to capture details of the flow in these areas.
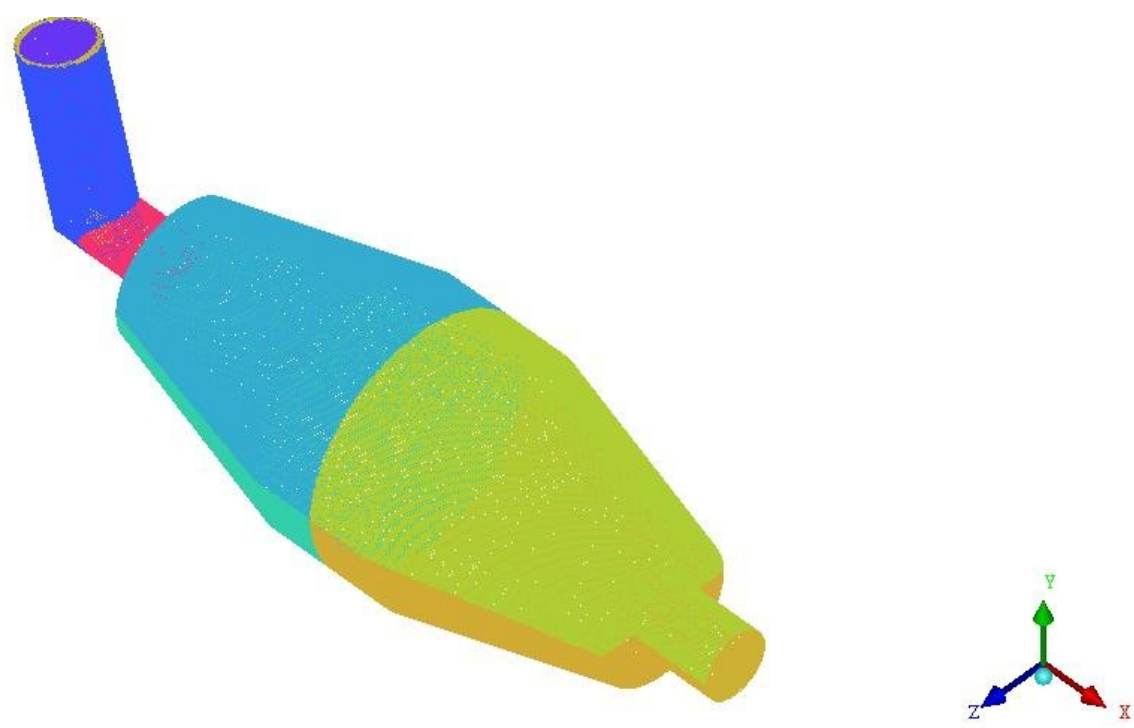

Figure 4.1: Geometry made in ICEM by ANSYS.

\subsection{Boundary Conditions}

To create a simulation with conditions used for the experimental procedure, the geometry was set using a mass flow outlet to simulate the varying inhalation flow rates between $7.5 \mathrm{~L} / \mathrm{min}$ and $60 \mathrm{~L} / \mathrm{min}$, as this is the point at which the airflow is for the experimental procedure. Using the area of the flow outlet and the density of air at $25^{\circ} \mathrm{C}\left(1.185 \mathrm{~kg} / \mathrm{m}^{3}\right)$, the following values were used to simulate the different flow 
rates:

\begin{tabular}{cc}
\hline Flow rate $(\mathrm{L} / \mathrm{min})$ & Mass flow outlet $(\mathrm{kg} / \mathrm{s})$ \\
\hline \hline 7.5 & 0.0001480 \\
15 & 0.0002963 \\
30 & 0.0005925 \\
60 & 0.001185 \\
\hline
\end{tabular}

Table 4.1: Flow rate and mass flow outlets

Using Equation 2.3 from Matida and Alhegagi [3], the flow from the nozzle was simulated transiently in order to replicate the downward angle of flow from an actuator with an initial velocity of $80 \mathrm{~m} / \mathrm{s}$. The boundary at the top of the canister was specified as a zero-gradient static pressure region, with a value of $0 \mathrm{~Pa}$ as the pressure difference for the experimental procedure did not show significant effect on the flow.

\subsection{Navier Stokes Equations}

Using ANSYS CFX, unsteady Reynolds Averaged Navier-Stokes (URANS) equations are used to study flow within the spacer. The Navier-Stokes equations averaged by separating the velocities of flow into a mean part and fluctuating part as follows;

$$
u_{i}=\overline{U_{i}}+u_{i}^{\prime}
$$

Where the averaged component, $\overline{U_{i}}$ is calculated in the following way;

$$
\overline{U_{i}}=\frac{1}{\Delta t} \int_{t}^{t+\Delta t} U_{i} d t
$$


In transient flow, unsteady RANS equations are used. In this form of modelling, values are ensemble averaged rather than time averaged, in order to study the behaviour of the flow at different time steps. By averaging the Navier-Stokes equations, less computational power is required than for other methods (such as large eddy simulations or direct numerical simulations), but the level of accuracy obtainable may be lower because of the unsteady parts of the flow being averaged out. When the averaged velocity is used to determine the continuity, the following is found;

$$
\frac{\partial \overline{U_{i}}}{\partial x_{i}}=0
$$

The Navier Stokes equations will also therefore become averaged and can be expressed as;

$$
\frac{\overline{\partial U_{i}}}{\partial t}+\overline{U_{j}} \frac{\partial \overline{U_{j}}}{\partial x_{j}}=-\frac{1}{\rho}+\frac{\partial \bar{P}}{\partial x_{j}}\left(\mu \frac{\partial \overline{U_{j}}}{\partial x_{j}}-\overline{u_{i} u_{j}}\right)
$$

Where the final term $\left(\overline{u_{i} u_{j}}\right)$ is the specific Reynolds stress tensor, $\tau_{i j}$. The Reynolds stresses are then given as;

$$
\rho \overline{u_{i} u_{j}}=\mu_{t}\left(\frac{\partial U_{i}}{\partial x_{j}}+\frac{\partial U_{j}}{\partial x_{i}}\right)-\frac{2}{3} \delta_{i j}\left(\rho k+\mu_{t} \frac{\partial U_{k}}{\partial x_{k}}\right)
$$

When $\delta_{i j}$ is the Kronecker delta, give as;

$$
\delta_{i j}=\left\{\begin{array}{cc}
1 & \text { when } i=j \\
0 & \text { when } i \neq j
\end{array}\right.
$$

In Equation 4.5, $k$ is used to represent the turbulent kinetic energy which is defined as;

$$
k=\frac{1}{2}\left(\overline{u_{i}^{2}}\right)
$$


Where $i=1,2,3$ The turbulent viscosity, $\mu_{t}$ is calculated in the following way;

$$
\mu_{t}=C_{\mu} \rho \frac{k^{2}}{\varepsilon} \quad \text { where } \varepsilon=\nu \frac{\partial u_{i} \frac{\partial u_{l}}{\partial x_{i}} \partial x_{l}}{2}
$$

Where $\varepsilon$ is the dissipation, and $C_{\mu}$ is a constant.

For simulations, the shear stress transport (SST) model was used as the eddy viscosity turbulence model. The SST model was developed because the available $k-\varepsilon$ and $k-\omega$ models were not sufficient for fully describing the behaviour of flow [50]. The $k-\omega$ model provides accurate predictions for near wall flows, but is not applicable to cases with high amounts of pressure, and outside of the boundary layer the model is sensitive to free stream conditions. Conversely, the $\mathrm{k}-\varepsilon$ model is not able to capture turbulence accurately at the boundary layers [51], but is accurate for the rest of the flow. Menter initially created a model to combine the $\mathrm{k}-\varepsilon$ and $\mathrm{k}-\omega$ models to use each for their prominent areas, using a blending function, $F_{1}$, to determine the model to be used, as the $\mathrm{k}-\varepsilon$ is most appropriate in the free stream area and the $\mathrm{k}-\omega$ model in the boundary layer [50].

The New Baseline model (BSL), so called because it gave a new approach to turbulence, incorporating both the $\mathrm{k}-\omega$ and $\mathrm{k}-\varepsilon$ models, uses the expression;

$$
\begin{gathered}
\frac{\partial(\rho k)}{\partial t}+\nabla \cdot(\rho \bar{U} k)=\nabla \cdot\left[\left(\mu+\frac{\mu_{t}}{\sigma_{k 3}}\right) \nabla k\right]+P_{k}+\beta^{\prime} \rho k \omega \\
\frac{\partial(\rho \omega)}{\partial t}+\nabla \cdot(\rho U \omega)=\nabla \cdot\left[\left(\mu+\frac{\mu_{t}}{\sigma_{\omega 3}}\right) \nabla \omega\right]+\left(1-F_{1}\right) 2 \rho \frac{1}{\sigma_{\omega 2} \omega} \nabla k \nabla \omega+\alpha_{3} \frac{\omega}{k} P_{k}-\beta_{3} \rho \omega^{2}
\end{gathered}
$$

It is also sometimes also referred to solely as the Baseline model. In this, the turbulent viscosity can be calculated as;

$$
\nu_{t}=\frac{\mu_{t}}{\rho}
$$


Although the BSL model improves the representation of flow, the shear stress of the results is over predicted. To compensate for this the SST model was developed where the turbulent viscosity is calculated in the following way, to better model the eddy-viscosity.

$$
\nu_{t}=\frac{a_{1} k}{\max \left(a_{1} \omega, S F_{2}\right)}
$$

$F_{1}$ and $F_{2}$ are blending functions that are dependent on the region of flow, and are calculated in the following way. Away from the wall the value of $F_{1}$ is zero, following the $\mathrm{k}-\varepsilon$ model, and otherwise uses the $\mathrm{k}-\omega$ model.

$$
F_{1}=\tanh \left(\arg _{1}^{4}\right)
$$

Where;

$$
\begin{gathered}
\arg _{1}=\min \left(\max \left(\frac{\sqrt{k}}{\beta^{*} \omega y}, \frac{500 \nu}{y^{2} \omega}\right) \frac{4 \rho k}{C D_{k w} \sigma_{\omega 2} y^{2}}\right) \\
C D_{k \omega}=\max \left(2 \rho \frac{1}{\sigma_{\omega 2} \omega} \nabla k \nabla \omega, 1.0 \times 10^{-10}\right)
\end{gathered}
$$

And for the blending function $F_{2}$ used for SST modelling;

$$
F_{2}=\tanh \left(\arg _{2}^{2}\right)
$$

Where;

$$
\arg _{2}=\max \left(\frac{2 \sqrt{k}}{\beta^{*} \omega y}, \frac{500 \nu}{y^{2} \omega}\right)
$$

In these equations, $\sigma_{k 1}, \sigma_{\omega 1}, \beta_{1}, \beta^{*}, \kappa$ and $\gamma$ are all constants fitted to the SST model, 
with the following values;

$$
\begin{aligned}
& \sigma_{k 1}=0.85, \quad \sigma_{\omega 1}=0.5, \quad \beta_{1}=0.0750, \quad a_{1}=0.31 \\
& \beta^{*}=0.09, \quad \kappa=0.41 \quad \gamma=\beta_{1} / \beta^{*}-\sigma_{\omega 1} \kappa^{2} / \sqrt{\beta^{*}}
\end{aligned}
$$

\subsection{Particle tracking}

For the simulations, 10,000 particles were released into the domain during the time that the puffer was actuated. Salbutamol was specified as a particle transport fluid. One way coupling was used so that the fluid with Schiller Naumann drag model, a model established by the flow around a sphere [52].

Particles were released in time from the nozzle of the actuator over a time of 100 ms, which is the amount of time taken for an actuation. A restitution coefficient valued unity was used on the walls of the spacer so that particles that landed on the walls would stick to it, meaning no bounding would occur as is assumed in actual use of a spacer.

For particles with small diameters (between $2.5 \mu \mathrm{m}$ and $5 \mu \mathrm{m}$ ) and with low density ratios, Lagrangian particle tracking is used to describe the motion of particles [53-58]. This is applicable to the mono-dispersley particles used in the particle tracking model. The modelling states that;

$$
\frac{d u_{p, i}}{d t}=\frac{u_{f, i}-u_{p, i}}{\tau_{p}}-g_{i}\left(1-\frac{\rho_{f}}{\rho_{p}}\right)
$$

And the relaxation time of the particle is expressed as;

$$
\tau_{p}=\frac{\rho_{p} d_{p}^{2}}{18 \mu \alpha}
$$


Where;

$$
\alpha=1+0.15 R e_{p}^{0.687}
$$

Where $R e_{p}$ is the particle Reynolds number. Each particle has an instanteous velocity, divided as in RANS into an average and a fluctuating component. This is defined as;

$$
u_{f, i}=\bar{u}_{f, i}+u_{f, i}^{\prime}
$$

In the simulations it is considered that the fluctuating component will be zero, and deposition that occurs is solely due to the average flow.

\subsubsection{Particle Size Distribution}

Initially, simulations were performed using a specified diameter of $3.53 \mu \mathrm{m}$, in accordance with the MMAD of salbutamol particles. As discussed in Section 2.3.2, particles released from a puffer are not mono-disperse. Different models can be applied in order to simulate the spread of particle diameters. In order to study the effect of using a diameter distribution, using the parameters given in Fink et al. [29] a normal diameter by mass was set whereby the mean, standard deviation and minimum and maximum diameters were set, allowing a variety of particle sizes to be released into the domain.

\subsubsection{Grid Convergence studies}

In CFD simulations error and uncertainty can stem from geometry simplification (known physical error) and from grid refinement and time steps (discretisation error). After creating the geometry in ICEM CFD, grid convergence studies were performed to ensure that an adequate number of elements and adequately small time steps were 
used for the simulations. The number of elements is required to be large enough to acquire results with a sufficient amount of accuracy suitable for the simulation, while at the same time minimising the computational power required, as larger numbers of elements require more processing power. The time steps should be small in order to save computational power but large enough that sufficient detail is produced in the results of the simulation.

Discretisation errors are addressed using spatial and temporal convergence analysis. Five grid sizes between 3,000,000 and 7,000,000 elements were used for spatial convergence analysis, as well as a 600,000 element mesh, in order to verify that an order of magnitude lower would not be suitable. In order to study grid convergence, steady state simulations were performed at an inhalation flow rate of $60 \mathrm{~L} / \mathrm{min}$, with the outlet velocity of the nozzle being $80 \mathrm{~m} / \mathrm{s}$ at a downwards angle of $6^{\circ}$. To determine the optimal mesh size, a characteristic of the simulation is selected and studied over the different mesh sizes. A grid is considered to be converged when the values of the selected characteristic converge, so that increasing the size of the mesh produces the same results, meaning that the smaller sized mesh can be used without losing accuracy in results [59-61]. The centre line velocity can be selected as in Hayase et al. [59], to perform this convergence analysis. The velocity through the centre line of the geometry and the horizontal vertical components of the inlet to the spacer were used to analyse the convergence, shown in Figure 4.2. Using these regions of interest it was possible to compare results for each grid size, showing how many elements are required for an accurate simulation.

Temporal analysis studies the number of time steps that are required to accurately represent the flow within the spacer, a factor important for transient simulations in ensuring that the full behaviour of the flow is captured. As the release of medication 


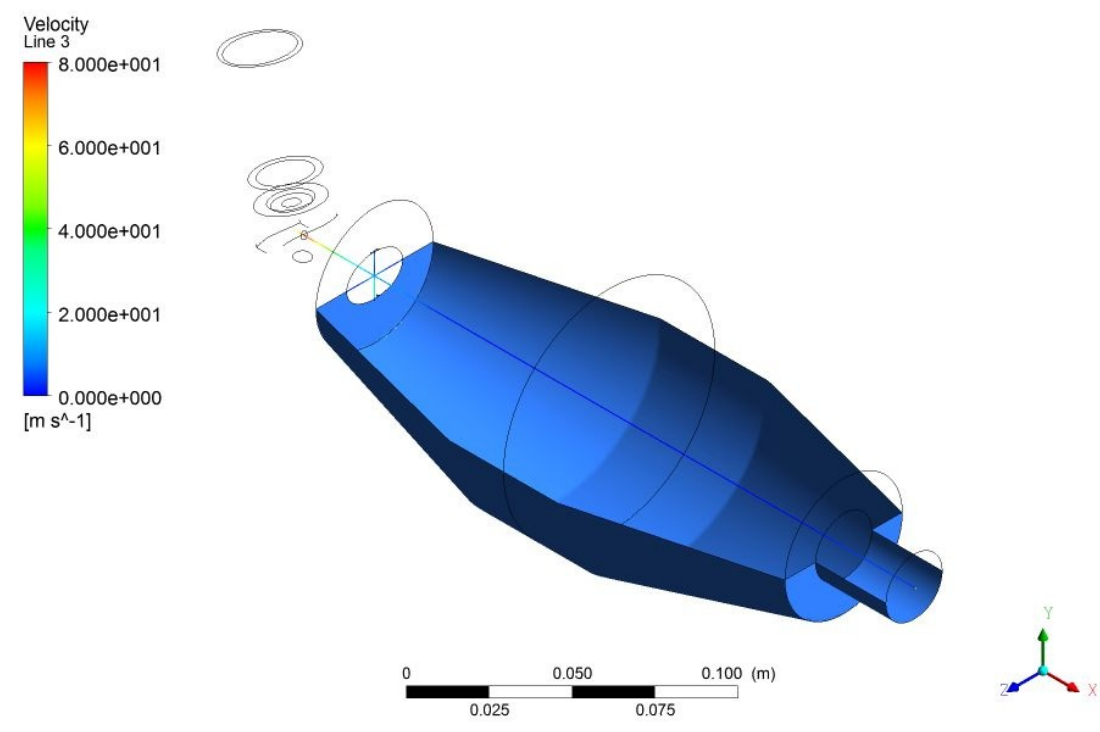

Figure 4.2: Areas of interest for grid convergence studies

takes a total time of $100 \mathrm{~ms}$ with fluctuations in the flow direction during the first 20 ms [32] it is necessary for an order of $1 \mathrm{~ms}$ as a maximum is used. Time steps of $1 \mathrm{~ms}$, $0.1 \mathrm{~ms}$ and $0.01 \mathrm{~ms}$ were used in the temporal convergence analysis, incorporating a downwards angle for the period of actuation. The outcome of each simulation can then be compared for similarities.

For transient analysis, to ensure stability and so that accurate results are being generated, the Courant number at each time step should be less than one. It is calculated in the following way:

$$
\text { Courant number }=\frac{U \Delta t}{\Delta x}
$$

For time steps of $0.1 \mathrm{~ms}$ the averaged Courant number consistently remained below one. It was found from analysis that $300 \mathrm{~ms}$ were required for simulations, as shorter periods of time would not allow the particles to travel fully through the spacer. 


\section{Chapter 5}

\section{Results}

In this chapter the results of the study are presented. The experimental results are presented first, before the uncertainty analysis to verify the significance of the results. The numerical studies are then presented, for transient results of monodisperse particle sizes and then for results with a diameter distribution added to more accurately represent the size of the particles. The purpose of these results is to determine the reason for deposition within the spacer, which is discussed in Chapter 6.

\subsection{Experimental results}

The objective of the experiments was to assess the deposition of salbutamol sulphate in different areas of a large volume spacer. To perform this assessment, an actuator was fired 20 times into a spacer, and the deposition on each quarter of the spacer was then assessed by separately rinsing each section in $20 \mathrm{ml}$ of $20 \%$ ethanol solution. The experiments were repeated a minimum of three times for five different flow rates $(7.5$, 15, 30, 45 and $60 \mathrm{~L} / \mathrm{min}$ ), which were set using a flow meter. UV spectrophotometry was used to take absorbance measurements of the solutions, and using a calibration 
curve the concentration of medication was found from the absorbance. Simultaneously, pressure readings were taken for the experiments to quantify the pressure loss in the spacer during actuations.

\subsubsection{Beer Lambert Calibration}

In order to quantify the amount of deposition of salbutamol sulphate on each part of the spacer, it was necessary to find the calibration coefficient linking the absorbtion to the concentration of a solution of the medication in $20 \%$ ethanol.

To perform calibration between the absorbance and the amount of medication deposited, 100 actuations of a Novo-Salbutamol puffer were fired into a beaker and weighed using Satorius scales, accurate to $0.001 \mathrm{mg}$. The results are shown in Table 5.1 .

\begin{tabular}{cc}
\hline & Mass $(\mathrm{g})$ \\
\hline \hline Mass of empty beaker & 108.1607 \\
Mass of beaker +100 actuations & 108.1716 \\
Mass 100 actuations & $1.09 \times 10^{-3}$ \\
Average actuation mass & $109 \times 10^{-6}$ \\
\hline
\end{tabular}

Table 5.1: Mass of 100 actuations of Teva Novopharm Salbutamol

Full results of concentration deposition measurements can be found in Appendix A. After actuating the medication into the beaker, quantities of $20 \%$ ethanol solution were added to produce solutions with known concentrations of salbutamol sulphate.

Absorbtion readings of the medication were taken at a fixed wavelength of 280 $\mathrm{nm}$ for a range of known concentrations, and following the procedure described in Section 3.6, the following calibration was found; 


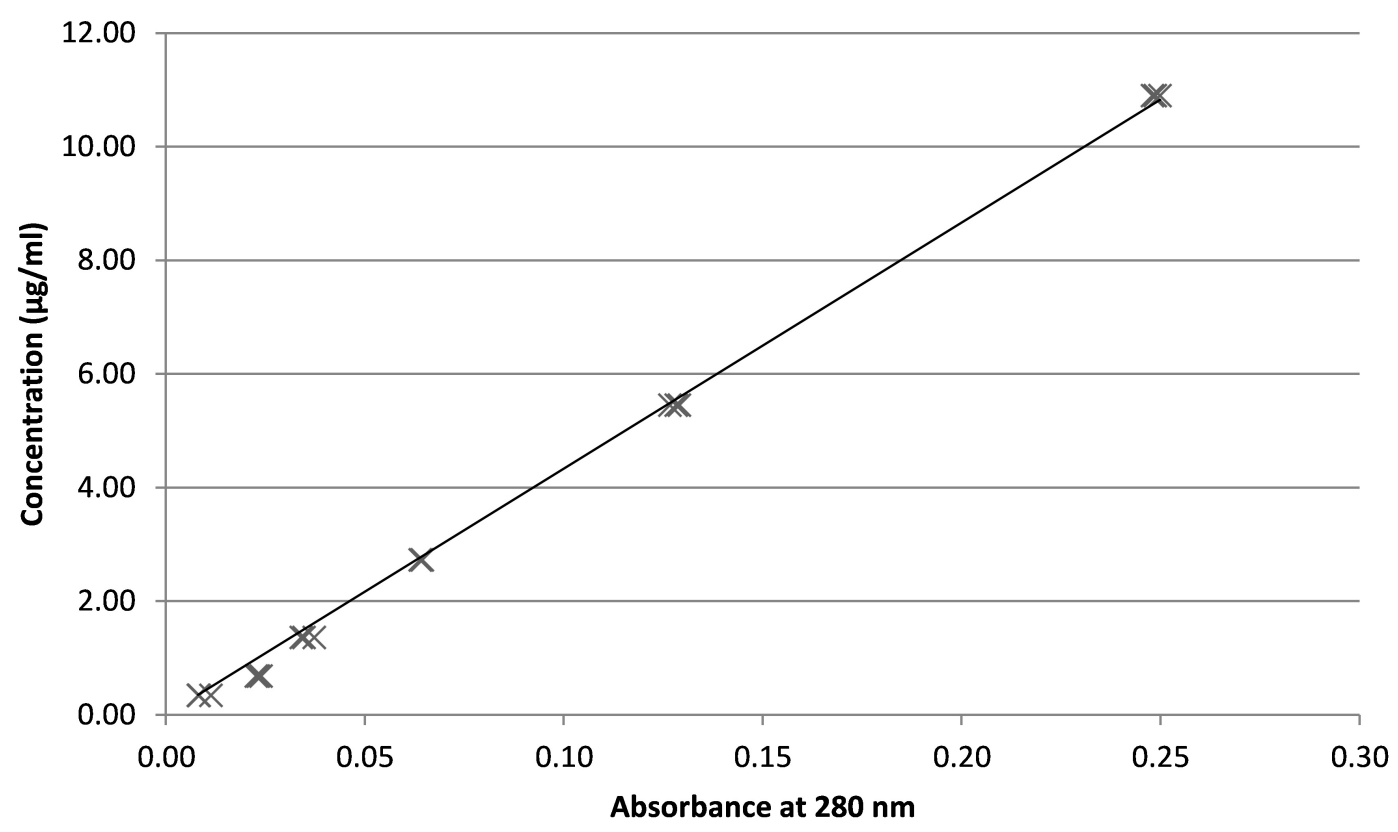

Figure 5.1: Beer-Lambert calibration curve

This gives the line of best fit with the following equation;

$$
\text { Concentration }=43.319 * \text { Absorbance }
$$

Where concentration is in $\mu \mathrm{g} / \mathrm{ml}$ and absorbance is taken at $280 \mathrm{~nm}$.

This relation between the two values is the Beer Lambert curve, described in Section 3.6. Using this, and with the knowledge that each part that was of interest was washed using $20 \mathrm{ml}$ of solution, it was possible to determine the amount of deposition. The calibration curve line of best fit gives the following coefficient of determination;

$$
R^{2}=0.9987
$$

This coefficient of determination shows that the calibration results closely follow the line of best fit with little error and deviation. 


\subsubsection{Deposition Measurements}

Deposition measurements were found to show trends when considering upper and lower deposition percentage, confirming the findings of Alhegagi and Matida [2] that an increased amount of deposition will occur on the lower half of the spacer. The effect of a patient increasing their inspiratory flow rate is given first. The percentage of the overall deposition within the spacer is then studied, comparing the upper and lower deposition percentages for each flow rate and comparing the proximal and distal deposition percentages for flow rates of $7.5 \mathrm{~L} / \mathrm{min}, 15 \mathrm{~L} / \mathrm{min}, 30 \mathrm{~L} / \mathrm{min}, 45 \mathrm{~L} / \mathrm{min}$ and $60 \mathrm{~L} / \mathrm{min}$. The error bars shown represent the standard deviation of each set of results.

\section{Actuation dosage}

From Table 5.1 it can be seen that the average released dose from the TEVA Novopharm actuator is $109 \mu \mathrm{g}$ per actuation $(9 \mu \mathrm{g}$ higher than the manufacturer's stated dose of $100 \mu \mathrm{g})$.

This dosage should result in an average of $2180 \mu \mathrm{g}$ of salbutamol sulphate being deposited overall during each trial. From the actual average measured deposition it can be predicted that $341.06 \mu \mathrm{g}$ of the actuation is lost due to being retained in the filter or because of errors in the wash procedure. From this, the bias error because of the wash procedure is taken to be $0.5 \%$ of the deposited dose, while an error of $0.2 \%$ is predicted for the bias error due to the absorbtion coefficient measurements.

\section{Flow rate effect on medication to the patient}

For all flow rates a substantially greater amount of medication occurred in the filter than in the spacer itself. Assuming that the medication released from the spacer will be respirable by the patient, this confirms that spacer usage is beneficial to a patient, 
as the amount of medication that reaches the patient for each flow rate is significantly higher than that for an actuator, as given by the literature. As the spacer reduces the velocity of the spray and gives particles more time to evaporate, this means that a large quantity of the medication in the filter would, if used by an asthmatic, be more likely to reach the lungs than a pMDI alone. From this it can be seen that spacers will increase lung deposition. It was found (as is shown in Figure 5.2) that the deposition in the filter increased with increasing inspiratory flow rate. This increased deposition

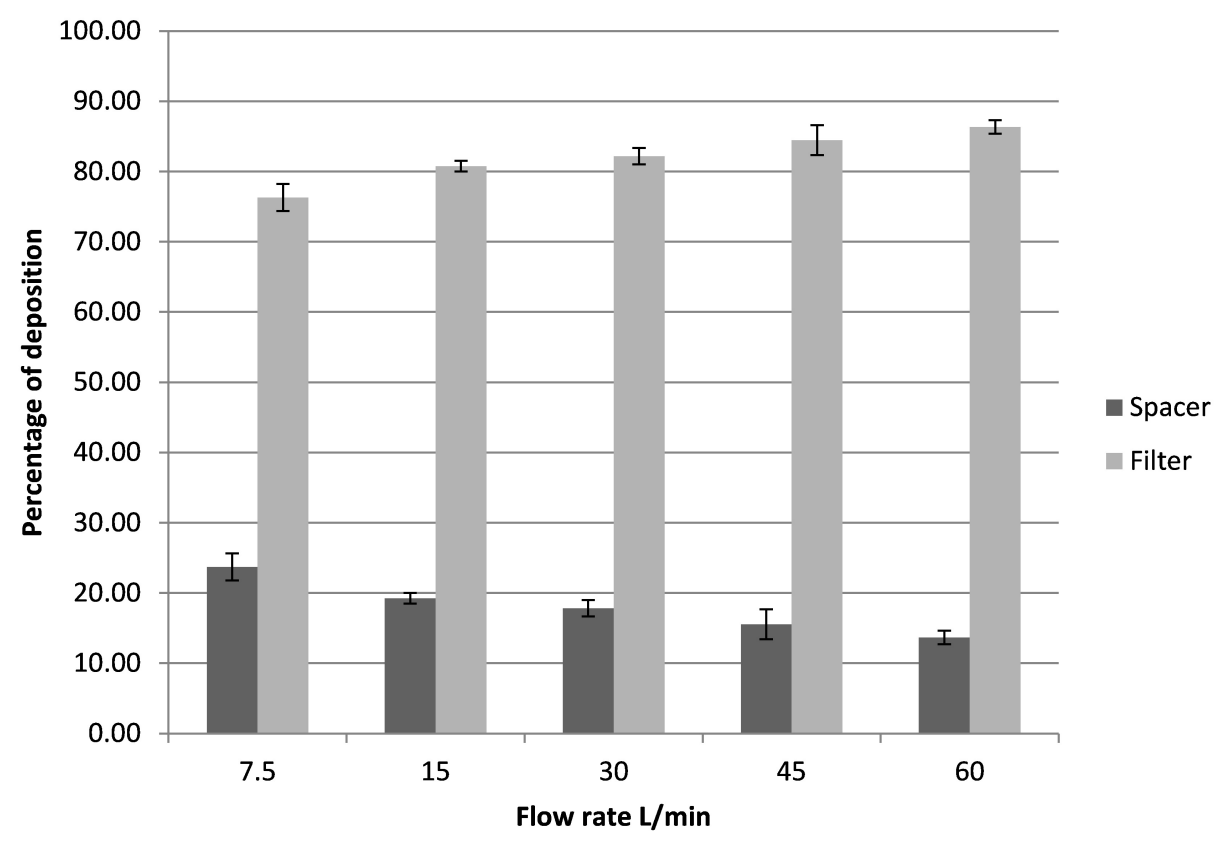

Figure 5.2: Percentage of spacer deposition

suggests that for a higher inhalation rate, more medication will be drawn out of the spacer, and therefore it is beneficial for patients using large volume spacers to breath in faster and deeper in order to achieve optimum conditions for drug delivery. 


\section{Vertical variation in deposition}

The vertical variation in deposition was measured for five different inhalation flow rates, 7.5, 15, 30, 45 and $60 \mathrm{~L} / \mathrm{min}$. From these experiments it was evident that, regardless of flow rate, a higher amount of medication would deposit on the lower half the spacer, reflecting the findings of Crosland [32]. As all flow rates display a greater quantity of medication depositing on the lower half of the spacer it can be assumed that the pMDI is spraying downwards for at least part of the actuation, and this downwards spray will cause impaction to occur on the spacer walls. From Figure

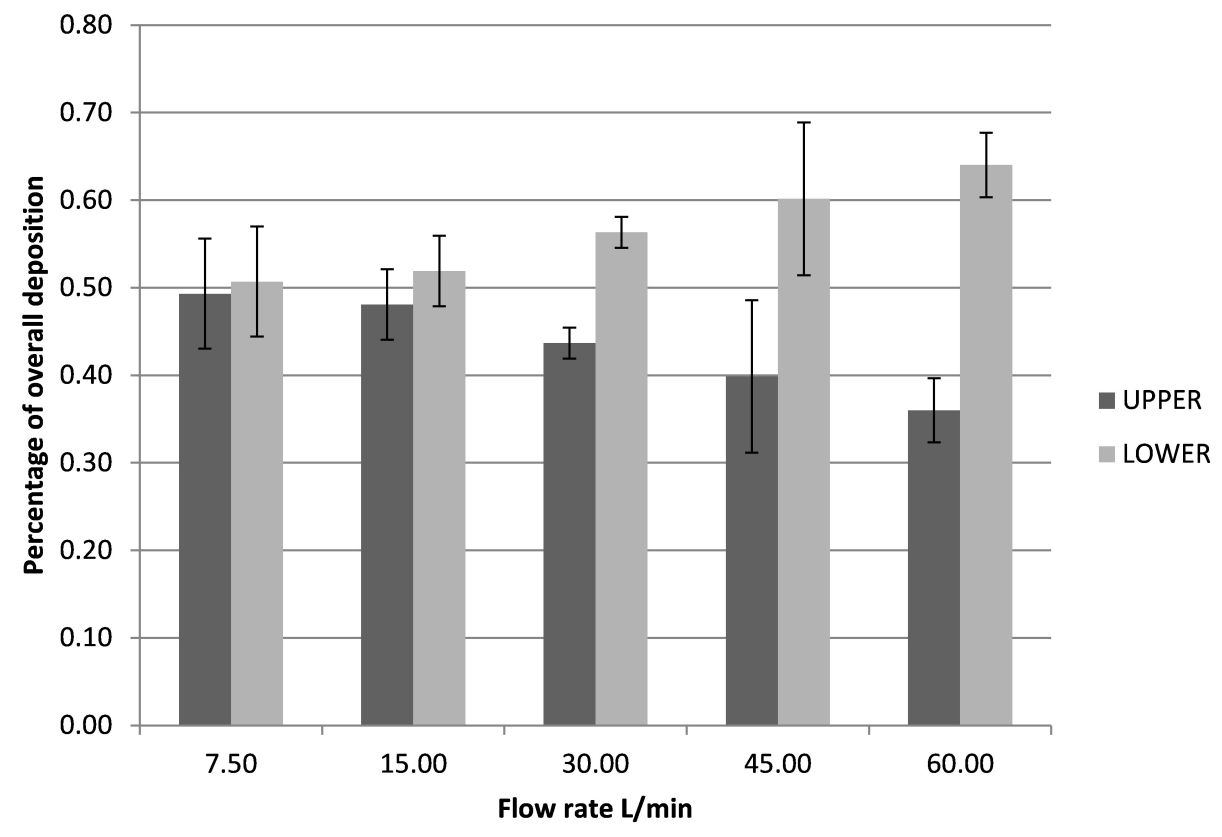

Figure 5.3: Upper and lower deposition

5.3 it can be seen that with increasing inspiratory flow rate, the ratio of the lower to upper medication deposition increases, showing that for higher inspiratory flow rates the deposition in the lower half of the spacer is greater. 


\section{Horizontal variation in deposition}

As for the vertical measurements, horizontal variation in deposition was studied for the five inspiratory flow rates specified in Chapter 3. The results varied dependent on the rate at which air was inhaled. For low inspiratory flow rates, a greater amount of deposition occured on the half of the spacer closer to the actuator. This deposition percentage decreased until $30 \mathrm{~L} / \mathrm{min}$, at which point a greater amount of deposition would occur in the distal part of the spacer. To study the causation of this asymmetric

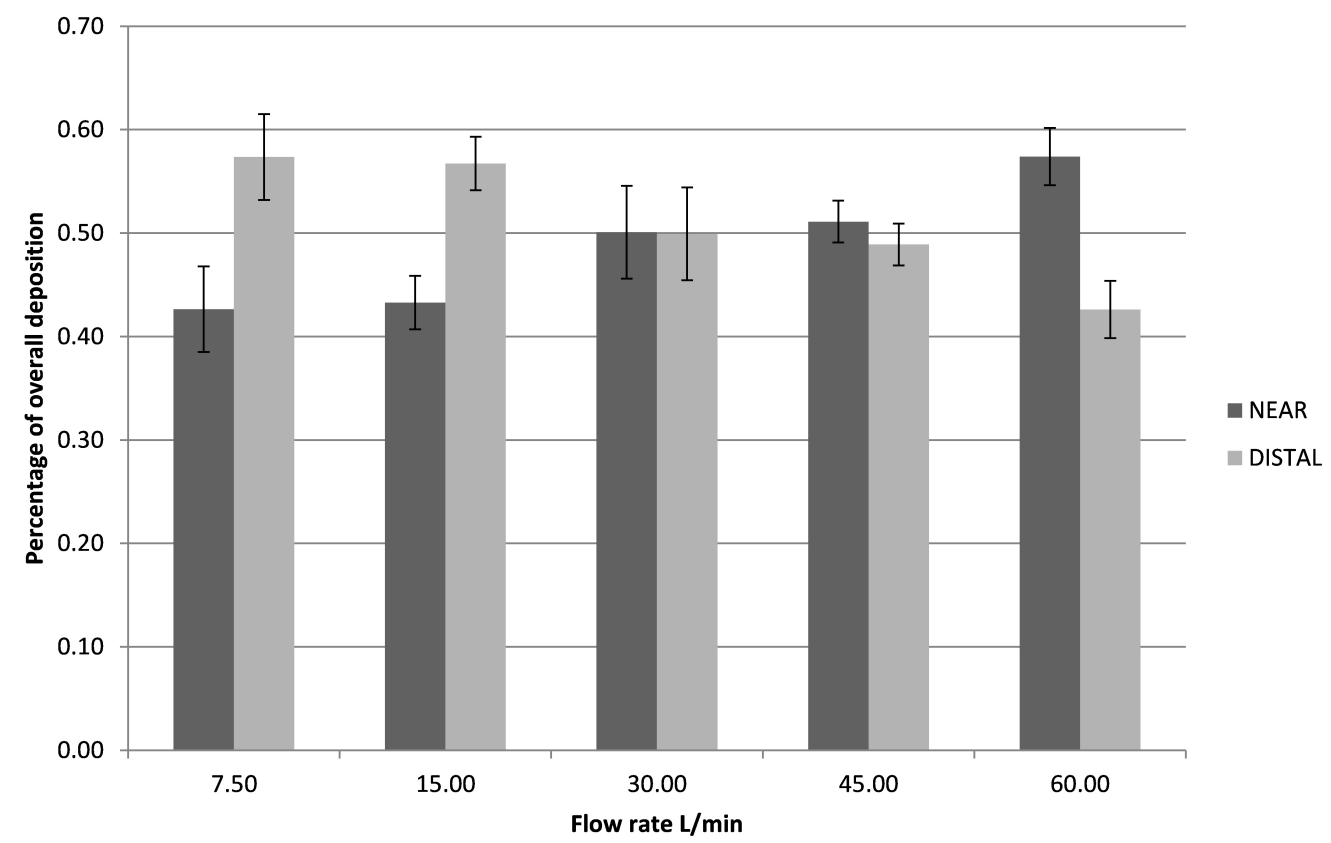

Figure 5.4: Proximal and distal deposition

variation of deposition, further studies should be carried out to verify these results and find potential causations of this deposition. 


\subsubsection{Error and uncertainty}

To determine the value of experimental results it was necessary to perform error and uncertainty analysis. This was performed according to ANSI/ASME standards [62]. Measurement errors can occur from calibration, data acquisition and data reduction errors [62].

\section{Bias error}

Tavoularis [63] states that bias errors are fixed or systematic, meaning that for all errors the bias will be the same. Bias errors can be eradicated from results to improve accuracy but are usually unknown and therefore have to be included. The total bias limit is stated as;

$$
b=\sqrt{\sum_{k=1}^{K} b_{k}^{2}}
$$

Where $k$ is the index for the bias and $b$ is the bias of the equipment.

Bias errors can be removed by carrying out experiments over a range of conditions and in different laboratories in order to assess the influence of the error. From the equipment used, the bias errors have been stated as $0.7 \%$ for the flow meter, and $0.25 \%$ for the pressure transducer.

The deposition fraction of medication on each section of the spacer and in the filter is to be measured. Using the experimental results, the deposition fraction of medication that reaches the filter can be calculated in the following way:

$$
D F=\frac{m_{\text {spacer }}}{m_{\text {spacer }}+m_{\text {filter }}}
$$

Where $m_{\text {spacer }}$ and $m_{\text {filter }}$ are the mass of medication within the spacer and within 
the filter respectively, that can be calculated using:

$$
m_{\text {spacer }}=V_{\text {spacer }} * A_{\text {spacer }} * k_{1}
$$

where $V_{\text {spacer }}$ is the volume of solution used in washing the spacer, and $A_{\text {spacer }}$ is the absorbance of the solution used to wash the spacer, and $k$ is the Beer-Lambert constant calculated in 5.1. The bias limit for the deposition fraction of medication that reaches the filter is found using:

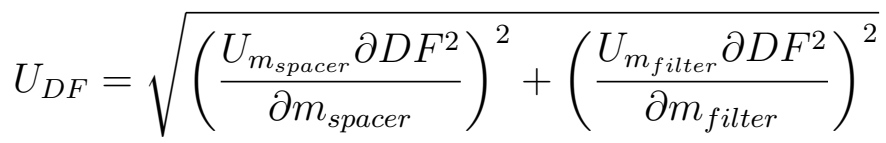

Using Equation 5.4, $\frac{\partial D F}{\partial m_{\text {spacer }}}$ can be found:

$$
\begin{aligned}
\frac{\partial D F}{\partial m_{\text {spacer }}} & =\frac{m_{\text {filter }}}{m_{\text {spacer }}+m_{\text {filter }}{ }^{2}} \\
& =\frac{D F(1-D F)}{m_{\text {spacer }}}
\end{aligned}
$$

In the same way, $\frac{\partial D F}{\partial m_{\text {filter }}}$ can be found:

$$
\begin{aligned}
\frac{\partial D F}{\partial m_{\text {filter }}} & =\frac{-m_{\text {spacer }}}{m_{\text {spacer }}+m_{\text {filter }}{ }^{2}} \\
& =\frac{-D F}{m_{\text {spacer }}+m_{\text {filter }}} \\
& =\frac{D F(1-D F)}{m_{\text {filter }}}
\end{aligned}
$$

Using Equations 5.7 and 5.8, $\frac{U_{D F}}{D F}$ can be found for the bias limit for deposition as:

$$
\frac{U_{D F}}{D F}=(1-D F) \sqrt{\left(\frac{U_{m_{\text {spacer }}}}{m_{\text {spacer }}}\right)^{2}+\left(\frac{U_{m_{\text {filter }}}}{m_{\text {filter }}}\right)^{2}}
$$


The total bias for the experiment involves both the bias limit of the equipment and the bias limit of the deposition fraction. Using the bias limit of the equipment as was given in Chapter 3, the bias error of the equipment is estimated as:

$$
b=\sqrt{\left(0.7^{2}\right)+\left(0.25^{2}\right)}=0.0074
$$

Combining the bias error of the equipment and the bias error of the deposition fraction, the total bias error for each reading can be calculated using:

$$
\text { Bias error }=\sqrt{\left(\frac{U_{D F}}{D F}\right)^{2}+\left(\frac{U_{\text {flowrate }}}{Q_{\text {flowrate }}}\right)^{2}}
$$

\section{Precision error}

Precision errors are non constant errors that arise because of measurement errors, and are sometimes referred to as random error. They cannot be disregarded in analysis because they have no set value. In order to find the precision error of the experiment, the standard deviation of the results needs to be found using;

$$
S_{\bar{X}}=\sqrt{\frac{\sum_{k=1}^{N}\left(X_{k}-\bar{X}\right)^{2}}{N-1}}
$$

The precision limit is found by taking into account the student t-test value for a $95 \%$ confidence interval with three samples. In this case, the value is 2.92 . The overall error of the experiments is then found, according to [62], using;

$$
U_{R S S}=\left[B^{2}+\left(t S_{\bar{X}}\right)^{2}\right]^{\frac{1}{2}}
$$


Where $U_{R S S}$ is the uncertainty, $B$ is the bias, $t$ is the 95 th percentile point for the student t-test (taken from tables) and $S_{\bar{X}}$ is the standard deviation.

\section{Errors in measurements for spacer and filter deposition}

Using the above methods of calculation, the following errors in measurement were found for the desposition in the spacer as a whole. Full error measurements are given in Appendix A.

Table 5.2: Measurement errors

\begin{tabular}{ccccc} 
Flow rate & DF & Bias Limit & Precision limit & Uncertainty \\
\hline \hline $7.5 \mathrm{~L} / \mathrm{min}$ & 0.2241 & 0.0238 & 0.0031 & 0.0608 \\
$15 \mathrm{~L} / \mathrm{min}$ & 0.1800 & 0.0250 & 0.0005 & 0.0333 \\
$30 \mathrm{~L} / \mathrm{min}$ & 0.1655 & 0.0254 & 0.0012 & 0.0429 \\
$45 \mathrm{~L} / \mathrm{min}$ & 0.1430 & 0.0260 & 0.0038 & 0.0672 \\
$60 \mathrm{~L} / \mathrm{min}$ & 0.1248 & 0.0265 & 0.0032 & 0.0625 \\
\hline
\end{tabular}

\section{$5.2 \quad$ Numerical results}

For the numerical analysis of the asymmetrical deposition within a spacer, both steady state and transient simulations were carried out. As described in Chapter 4, particle size distribution and its effect on the deposition within the spacer (and the characteristics of particles to reach the patient) were included in simulations to attempt to find a more accurate simulation of a large volume spacer used with a pMDI. 


\subsubsection{Grid Convergence studies}

Grid convergence studies were performed to ensure that the mesh used was fine enough to produce accurate results without requiring extensive time and power to perform. As discussed in 4.4.2, grid sizes between 3,000,000 and 7,000,000 tetrahedral elements were tested using a steady state simulation, as well as using an order of magnitude lower $(600,000$ elements) in order to determine that this was a suitable range to use. As can be seen from all lines of reference, 600,000 elements produced much different results and would not be in a suitable size range for this simulation.

As can be seen in Figures 5.5, 5.6 and 5.7, a mesh with 5,000,000 elements would produce similar results to meshes with a greater number of elements, but would require a smaller amount of time to converge, and therefore 5,000,000 elements were considered sufficient for analysis. The results of this analysis are as follows;

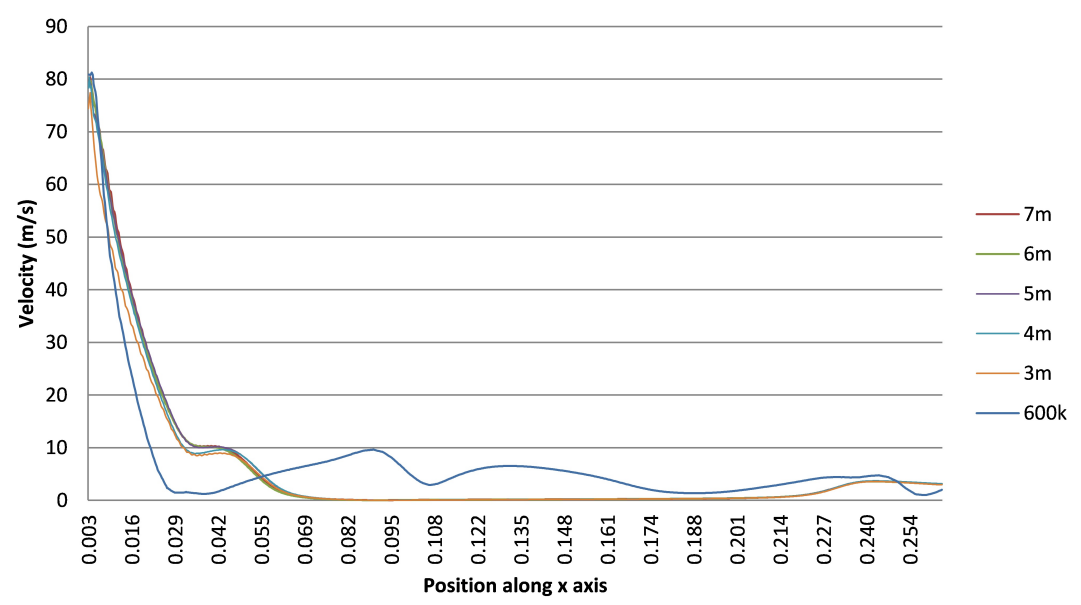

Figure 5.5: Velocity along x-axis

From the temporal analysis it was found that $0.1 \mathrm{~ms}$ per time step was sufficient, as $1 \mathrm{~ms}$ time steps would not fully capture results and $0.01 \mathrm{~ms}$ time steps would require an inefficient amount of time for each simulation, where $0.1 \mathrm{~ms}$ time steps 


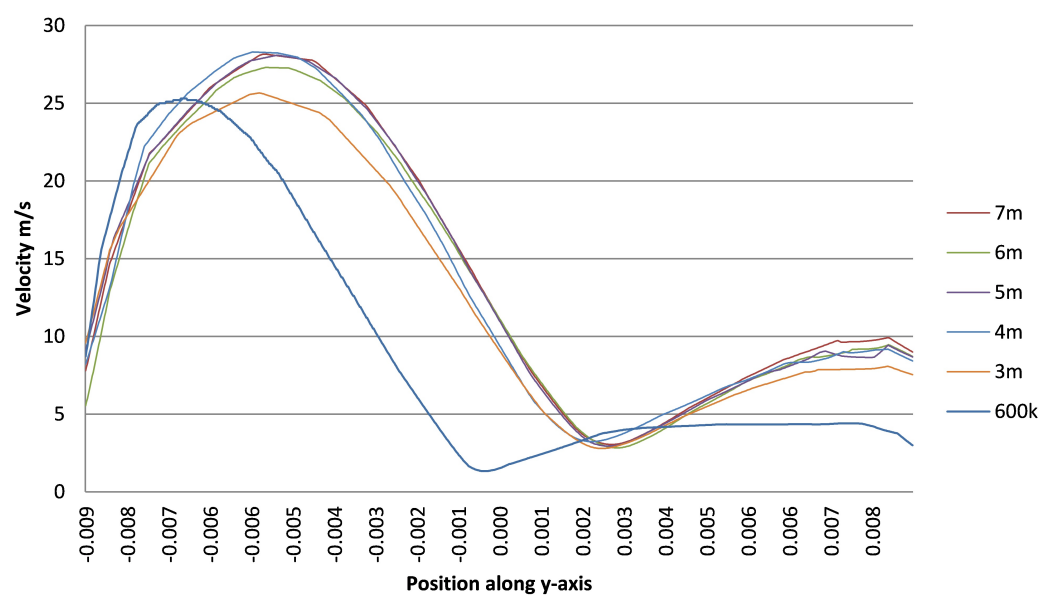

Figure 5.6: Velocity along y-axis

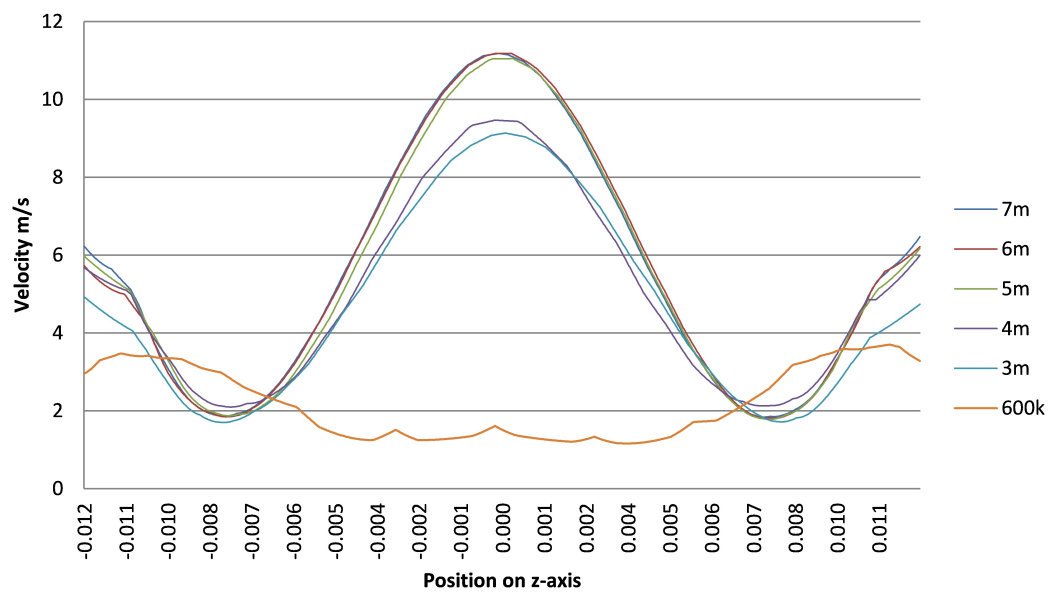

Figure 5.7: Velocity along z-axis

obtain sufficient detail.

As previously mentioned, the Courant number of a simulation provides a monitor for the accuracy and stability of transient results. For time steps of $0.1 \mathrm{~ms}$ the averaged Courant number consistently remained below one. It was found from analysis that $450 \mathrm{~ms}$ were required for simulations, as this amount of time would allow particles to fully travel through the spacer after actuation. 


\subsubsection{Simulation results}

Simulations were carried out at $15 \mathrm{~L} / \mathrm{min}, 30 \mathrm{~L} / \mathrm{min}$, and $60 \mathrm{~L} / \mathrm{min}$ to represent the trend in increasing inspiratory flow rate for the spacer, and to compare to the experimental results of flow within a large volume spacer. Using URANS and particle tracking it was possible to extract the information of where particles deposited within the spacer, allowing the total deposition at each surface to be recorded.

\subsubsection{Velocity in the spacer}

By using a transient simulation it was possible to simulate the fluctuating flow of the medication from the spacer, based on the findings of Crosland [32], as shown in the following images:

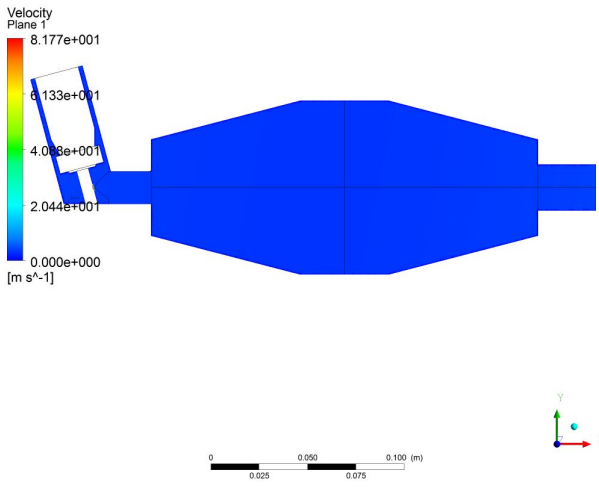

(a) $0.001 \mathrm{~s}$
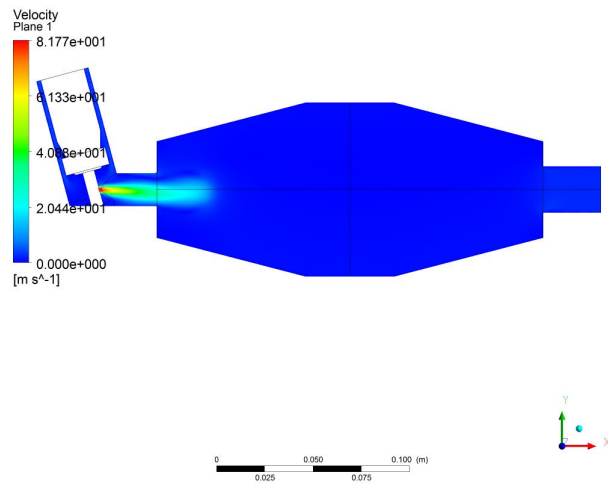

(b) $0.005 \mathrm{~s}$ 

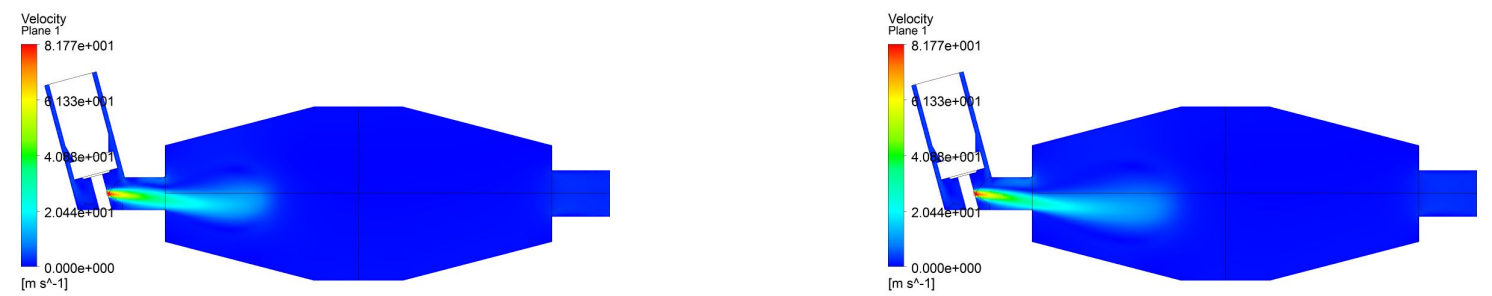

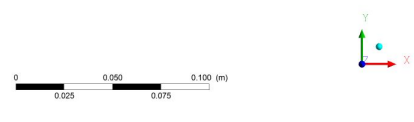

(c) $0.010 \mathrm{~s}$
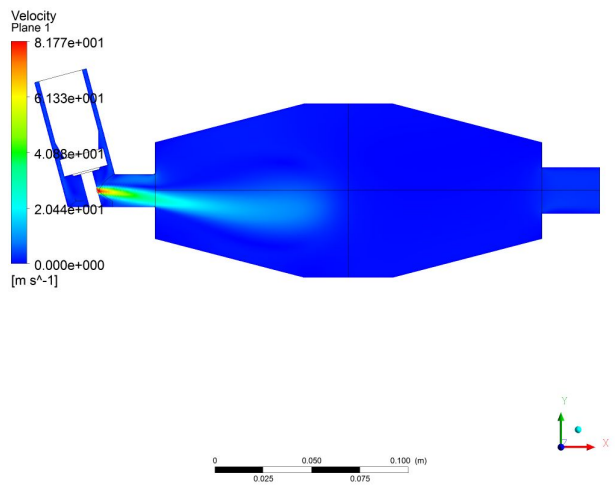

(e) $0.020 \mathrm{~s}$
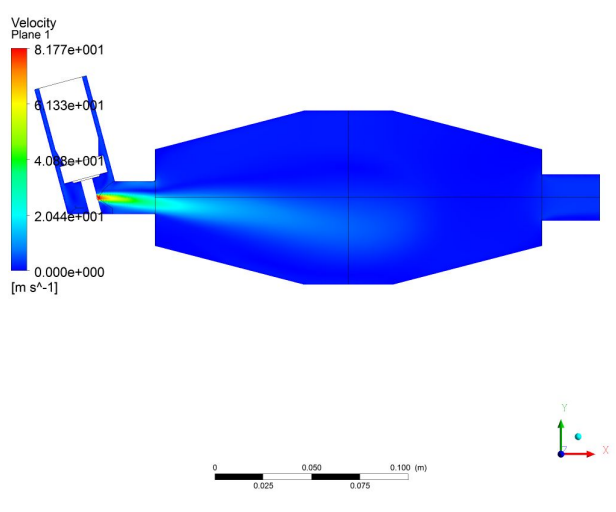

(g) $0.040 \mathrm{~s}$

$\rightarrow$

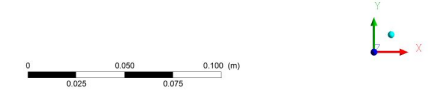

(d) $0.015 \mathrm{~s}$
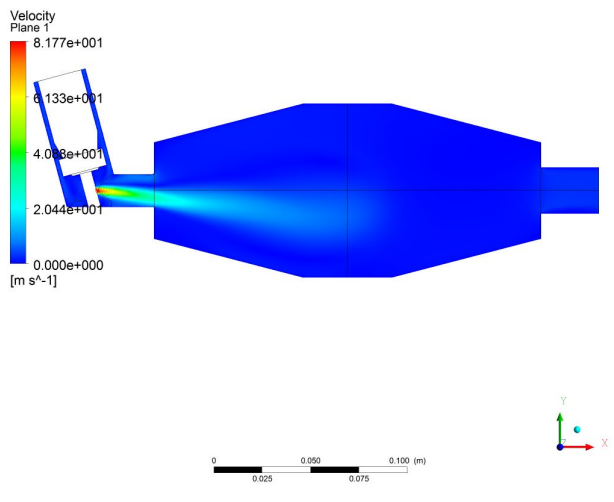

(f) $0.030 \mathrm{~s}$

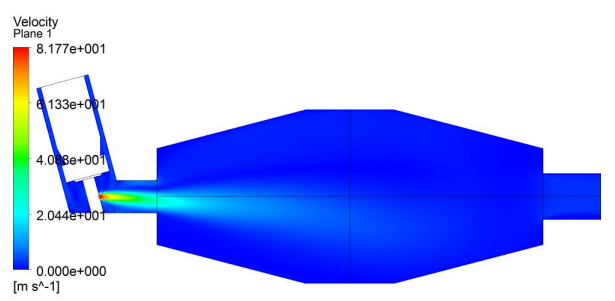

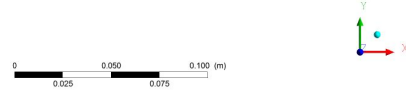

(h) $0.060 \mathrm{~s}$ 


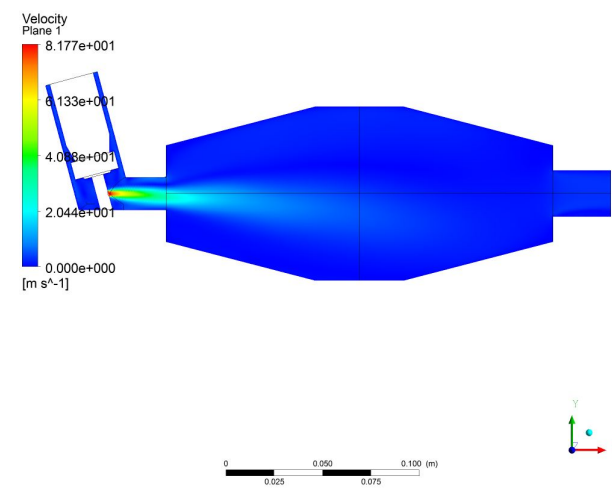

(i) $0.080 \mathrm{~s}$
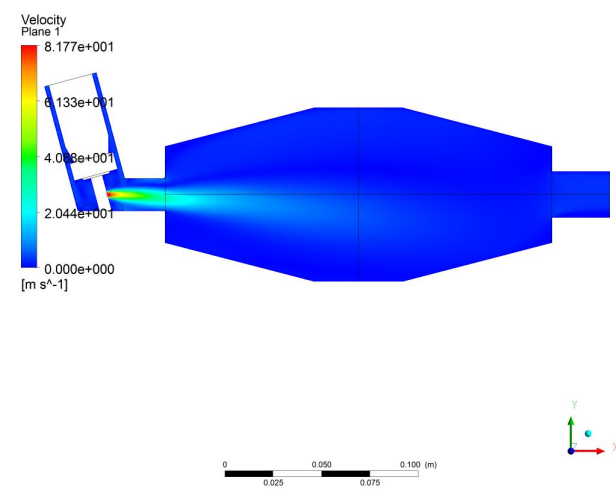

$\stackrel{\lfloor}{\longrightarrow}$

(j) $0.100 \mathrm{~s}$

Figure 5.6: Fluctuating spray angle in time

Previous studies have shown that the oropharyngeal deposition that can occur with the use of a pMDI is a source of reduction to the total amount of medication that will reach a patient, and impaction in this region can be due to the high velocity of the medication plume released from the pMDI. Spacer use increases the distance between the patient and the actuator and in this distance the velocity of the medication plume reduces significantly. Figure 5.6 shows this throughout the actuation of the puffer. Figure 5.7 shows how the velocity along the centre of the spacer decreases significantly with distance from the actuator nozzle, and will reduce to a minimum after half of the length of the spacer $(10 \mathrm{~cm})$. This shows that the use of a spacer would be beneficial for the reduction of oral and orophyrangeal deposition from inertial impaction, as the increased distance will result in slower moving particles being delivered to the mouth, which are easier to inhale. When studying the turbulent kinetic energy within the spacer, it can also be seen that turbulent kinetic energy is prominent near the actuator and decreases through the spacer, as shown in Figure 5.8. 
93

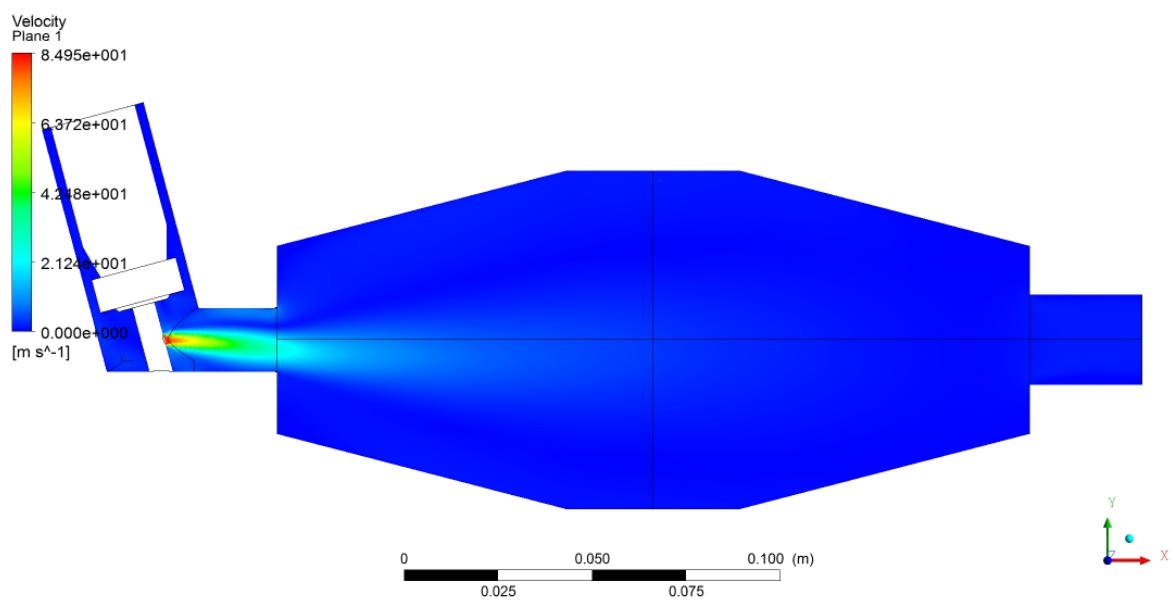

Figure 5.7: Velocity through spacer

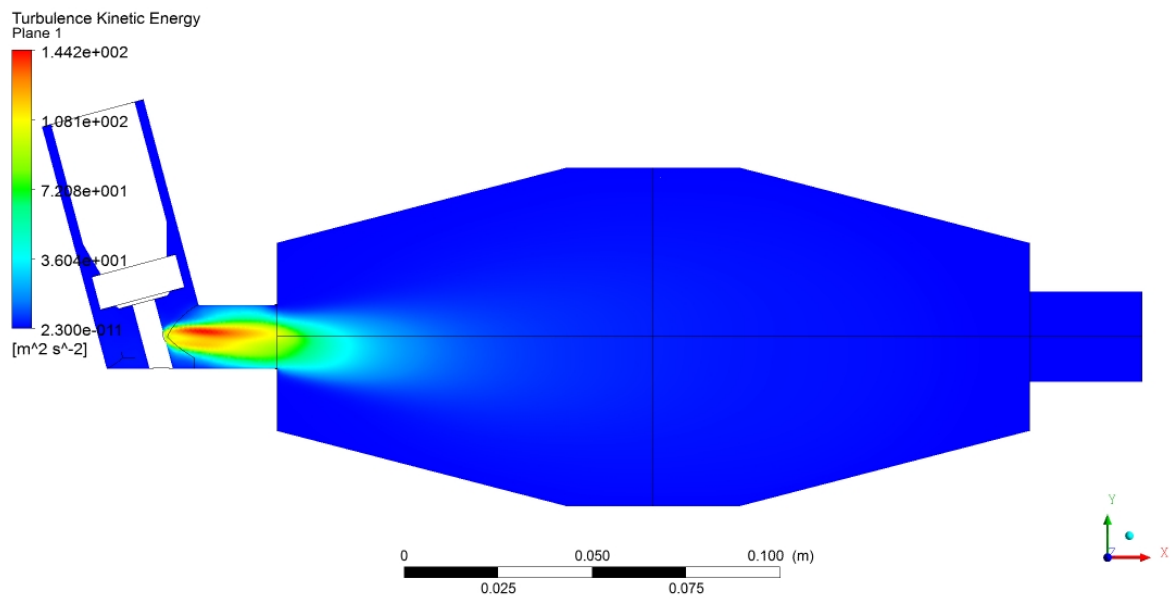

Figure 5.8: Turbulent kinetic energy during actuation 


\subsubsection{Particle tracking}

To verify the conditions used in simulations, results were compared to the experimental data that was obtained regarding the deposition of medication within the spacer. To achieve this, 10,000 particles were released into the domain from the actuator nozzle and their paths were tracked through the spacer.

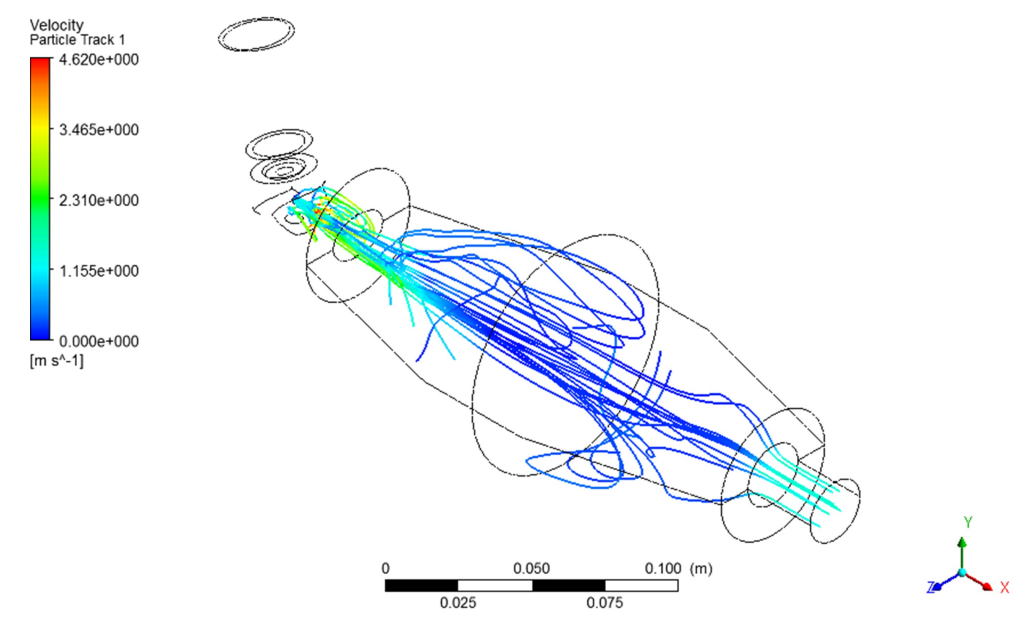

Figure 5.9: Mean flow particle tracking for $30 \mathrm{~L} / \mathrm{min}$ flow

The point at which the medication would deposit in the spacer could then be found using in house FORTRAN to extract the final position of each particle. The results for deposition at $30 \mathrm{~L} / \mathrm{min}$ are shown in 5.10 .

Mean flow tracking and turbulent tracking were compared to the experimental results in order to find the most suitable modelling option. Figure 5.11 shows the particle paths for turbulent tracking in simulation, meaning that each particle is considered to be interacting with eddies while in motion through the spacer.

From Figure 5.12 it can be deduced that the particle deposition is not due to turbulence in the flow but due to the downwards spray angle of the actuator, as turbulent tracking released a significantly smaller amount of medication to the patient 


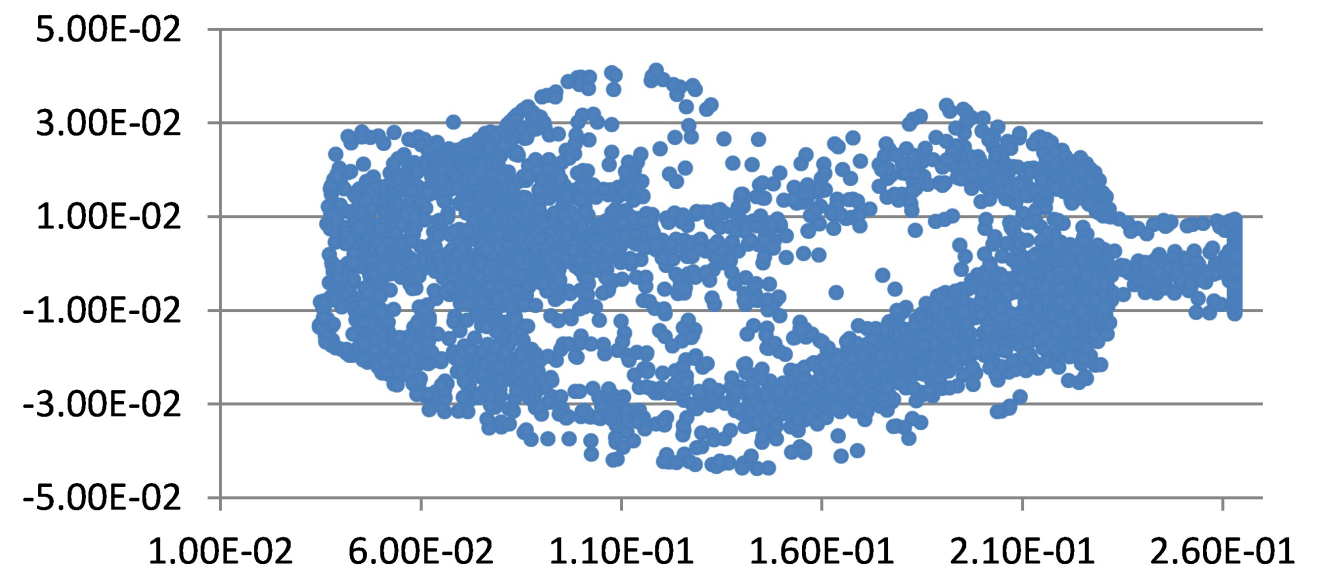

Figure 5.10: Side view for article deposition for $30 \mathrm{~L} / \mathrm{min}$ flow

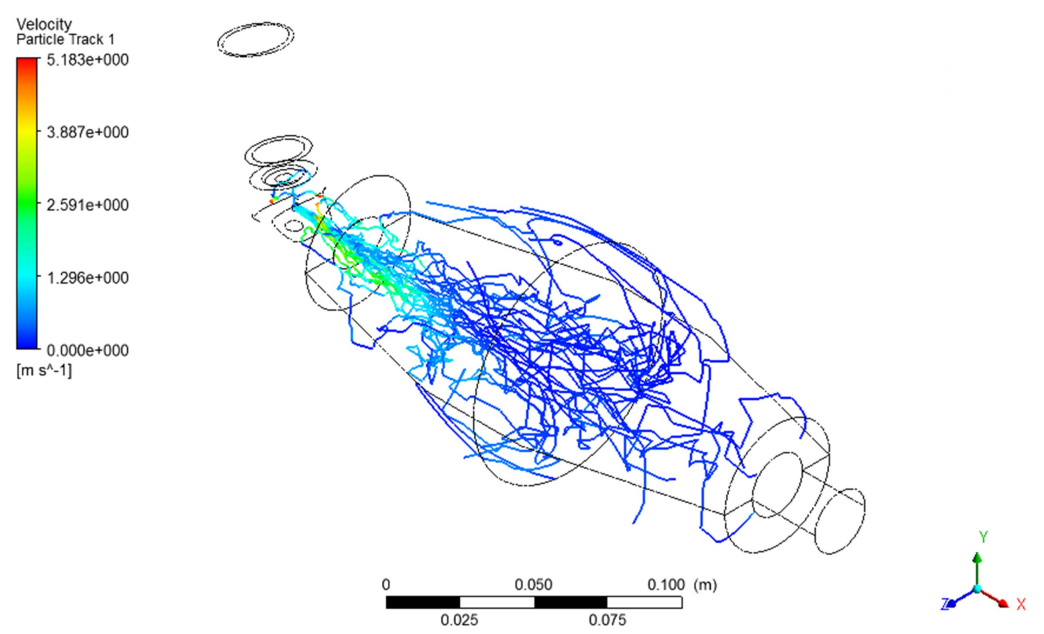

Figure 5.11: Turbulent particle tracking for $30 \mathrm{~L} / \mathrm{min}$ flow 
than mean flow tracking, meaning that a larger quantity of medication will attach to the walls of the spacer. This information reflects what was found in Alhegagi and Matida [2], which suggested that the deposition within a spacer was more likely to be as a result of inertial impaction on the walls of the spacer, rather than due to turbulence from the flow. Furthermore, the results of the mean flow tracking show

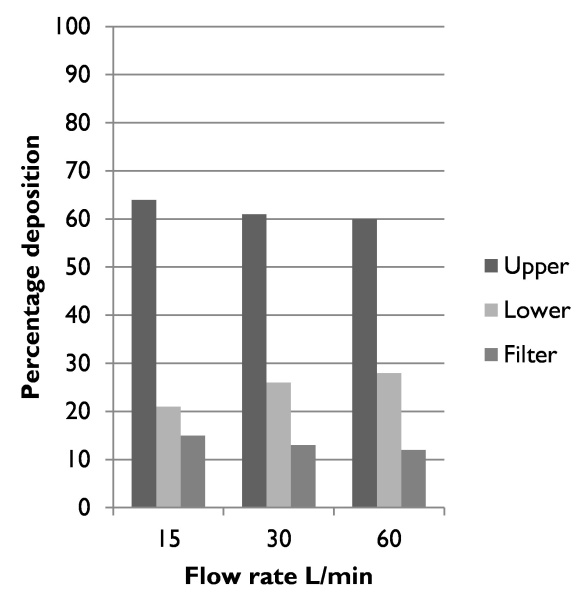

(a) Turbulent tracking

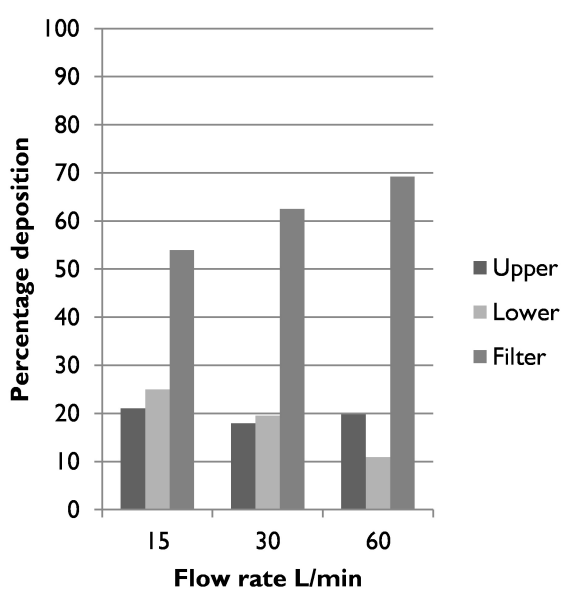

(b) Mean flow tracking

Figure 5.12: Comparison between turbulent and meanflow tracking in ANSYS CFX

that a larger amount of medication will, generally, reach the patient than will stay in the spacer, and this amount will increase with increasing inspiratory flow rate, which is a similar finding to experimental results.

The results show that lower flow rates will draw significantly less medication from the spacer, and that higher inspiratory flow rates are beneficial for use with a spacer.

The following figures demonstrate the comparison between CFD and experimental results for upper and lower deposition.

At a flow rate of $30 \mathrm{~L} / \mathrm{min}$, the simulation was also carried out with the medication being released without fluctuation, at a steady velocity of $80 \mathrm{~m} / \mathrm{s}$ in the x-direction. 


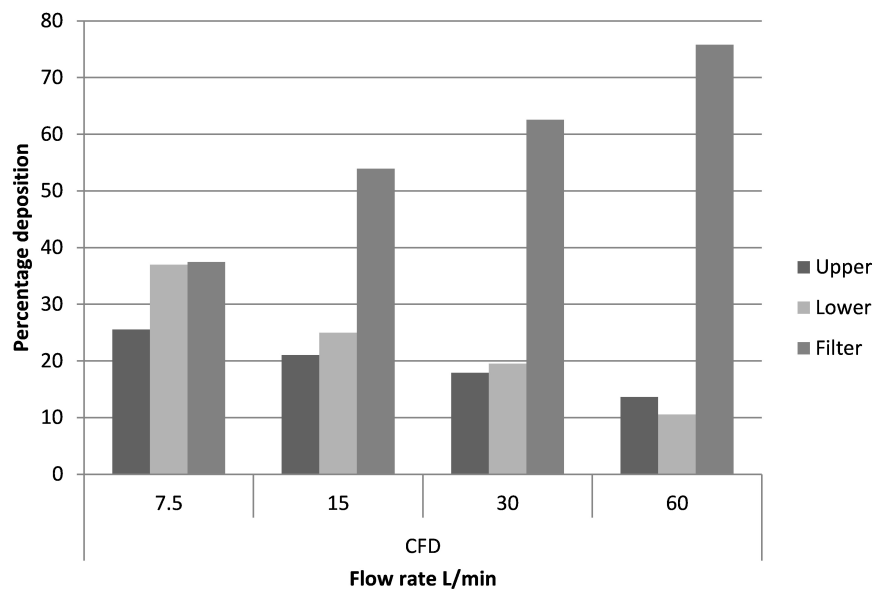

Figure 5.13: Mean flow tracking deposition results (CFD)

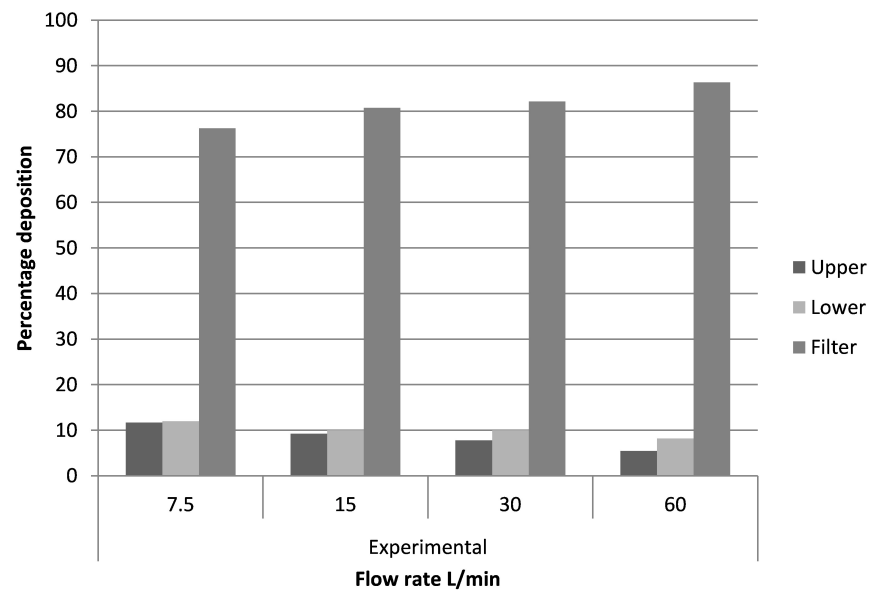

Figure 5.14: Experimental results for deposition 
While deposition was lower within the spacer itself, the deposition on the lower half of the spacer decreased significantly, which suggests that the fluctuation in the actuation flow is appropriate to use as experimental results show that deposition on the lower half of the spacer is higher, shown in the following figure:

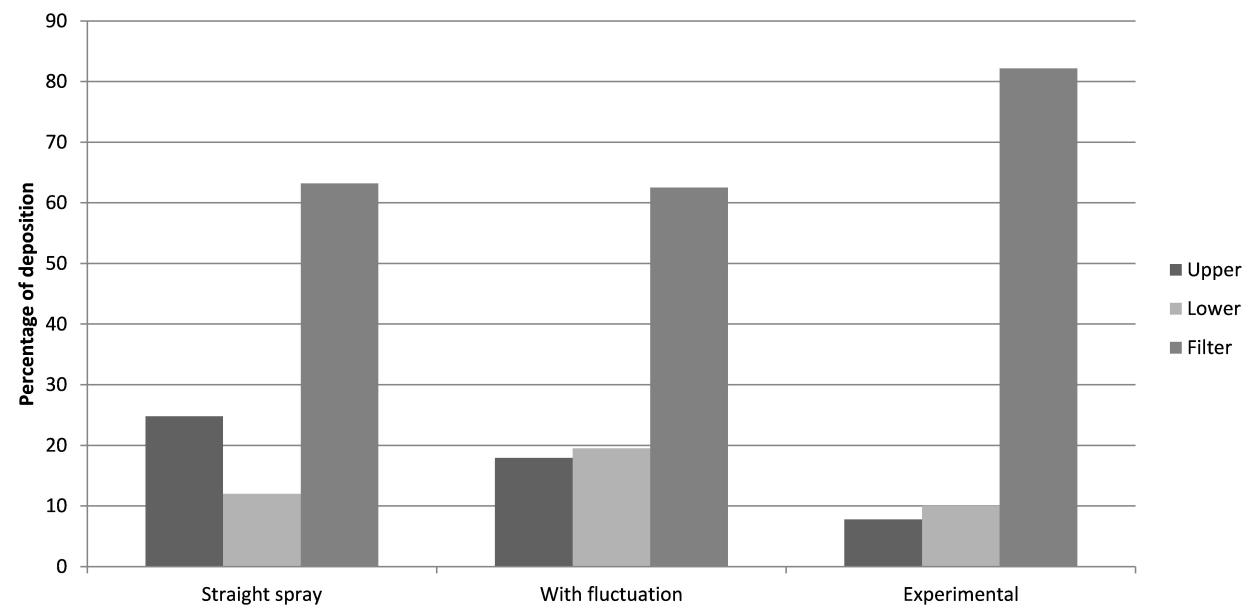

Figure 5.15: Comparison of spray angles

\subsubsection{Particle size distribution}

A diameter distribution was implemented for flow with an inhalation flow rate of 30 $\mathrm{L} / \mathrm{min}$ in order to compare the results to uniform diameter medication particles. Using the parameters defined in Chapter 2, it was found that with a diameter distribution of particles the number of particles that reaches the patient slightly increases. This produces closer results to the experimental results for a flow rate of $30 \mathrm{~L} / \mathrm{min}$, as shown: Research shows that medication attaching to the walls of a spacer can be beneficial as it reduces the amount of large particles inhaled, which have a diameter to great to be respirable. As shown in Figure 5.17, this method of modelling did not reflect this, and therefore additional methods must be implemented to reflect the 


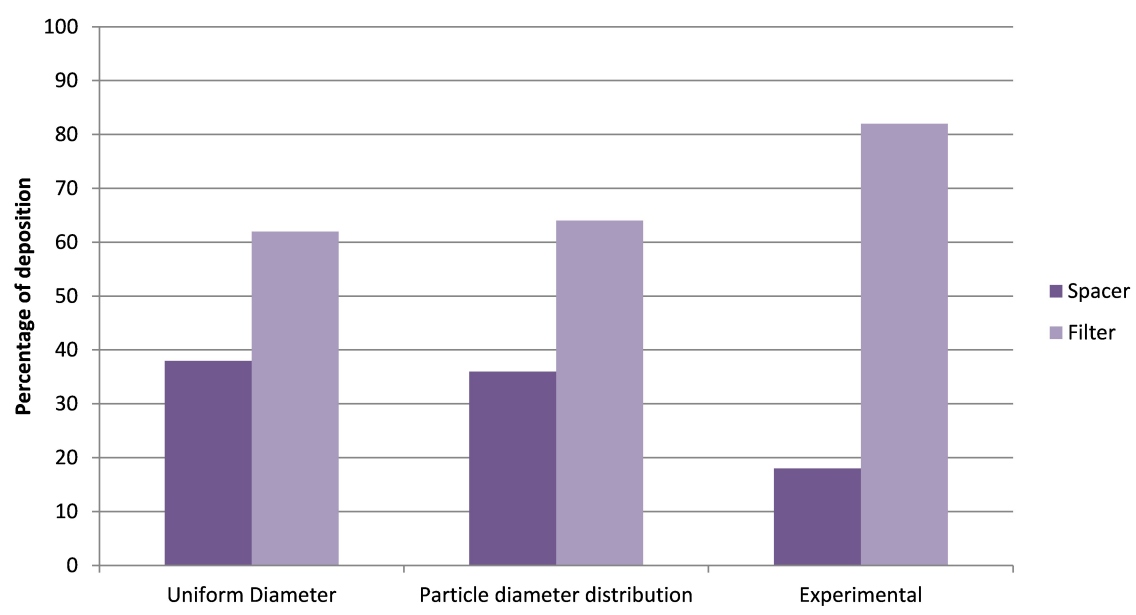

Figure 5.16: Comparison of uniform diameter and diameter distribution with experimental results

behaviour of different sized particles inside a spacer.
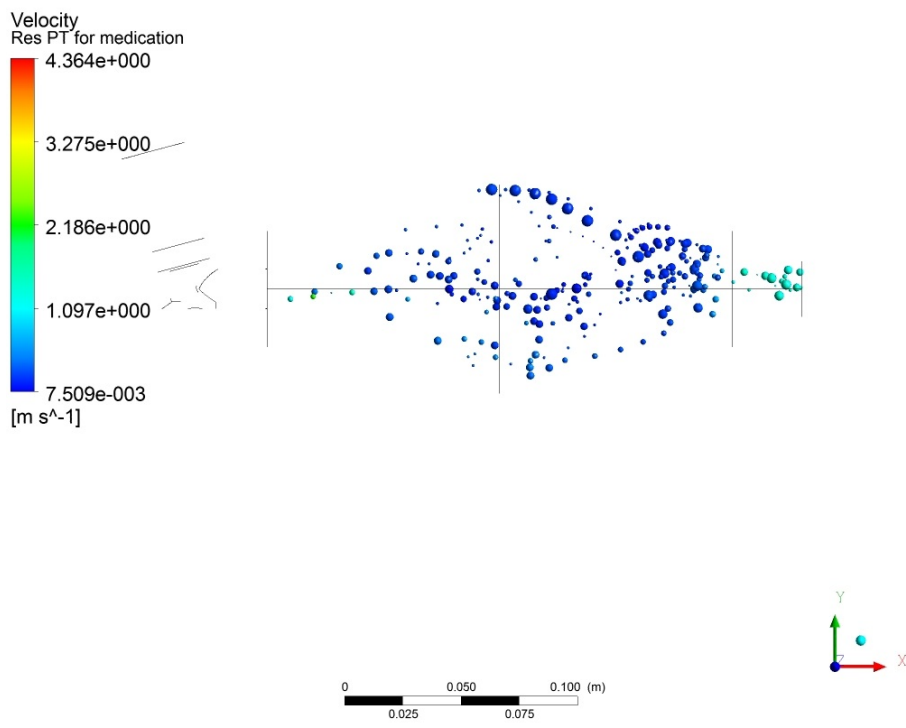

Figure 5.17: Particle diameter distribution 


\section{Chapter 6}

\section{Discussions, Conclusions and Future Work}

This thesis has developed and implemented previous work performed in Alhegagi and Matida [2], Fiset [4], Matida and Alhegagi [3] and Crosland [32] in order to assess the deposition of the asthma medication, salbutamol sulphate, in a large volume spacer. The $750 \mathrm{ml}$ capacity Volumatic ${ }^{\mathrm{TM}}$ spacer has been assessed experimentally at different flow rates to determine the impact of inhalation flow rates on regional deposition of medication within the spacer, and to quantify how much medication reaches a patient when using this spacer.

A geometry to represent the spacer has been created using ICEM CFD software, and, using ANSYS CFX, the performed experiments have been simulated to replicate the results in order to determine the reason for the deposition. In this thesis transient results have been acquired, encorporating the downwards spray angle from the pMDI reported in Crosland [32]. A Rosin Rammler size distribution has also been implemented for the different diameters of particles that occur in a spray. 


\subsection{Discussions and conclusions}

The results of this study confirmed the findings of Alhegagi and Matida [2] and Matida and Alhegagi [3], that when using a spacer with an actuator for the delivery of asthma medication, a higher percentage of the deposition will occur on the lower half of the spacer. It has also been found that there is a trend in the horizontal region of deposition within the spacer for varying inhalation flow rates, with a higher percentage of medication depositing on the half of the spacer at higher inhalation flow rates.

From the results it can also be seen that, when using a spacer such as the Volumatic TM spacer, a higher inhalation flow rate is beneficial for increasing the amount of medication that will reach the patient. This finding was complemented by the results of the numerical simulations, whereby a higher inspiratory flow rate resulted in a higher percentage of medication reaching the patient when using mean flow tracking.

When using turbulent tracking in ANSYS CFX it was found that significantly less medication would reach the patient than the experimental results. As mean flow tracking produced results closer to the physical experiments it can be deduced that the deposition on the walls of a large volume spacer is mainly due to inertial impaction.

\subsection{Future Work}

There are a number of ways that this research could be developed further, in both physical experimentation and in the CFD simulations. The following are recommendations for improving the experimental results of this thesis:

1. The experiments performed use a vacuum pump in order to simulate a constant inspiratory flow. As patient inhalation is broken up into long, deep breaths 
where the flow rate is similar to a partial sine wave, the method of breath simulation could be improved. A breathing machine could be used to simulate this, or a valve which could be accurately and easily changed from one flow rate to another in a short period of time, could be used and the flow rate could be manually changed during simulation.

2. More spacers could be considered experimentally using the same method to assess whether the regions of deposition are volume or shape dependent.

3. Different medication could also be tested in the same way, and with different propellants, to assess the effect that this may have.

4. The spacer itself could be further divided in order to enhance the precision of where medication deposition is occurring in the spacer.

In order to enhance the results of the CFD simulations the following ideas could be implemented:

1. The domain could be initialised, as though the patient was already breathing in when the actuation occurred.

2. More models could be used to simulate the distribution of particle diameters to assess the impact that this would have on results, and if a more accurate simulation of the released dose could be achieved.

3. Modifications could be made to the mesh in an aim to eliminate any simplifications of the model.

4. Large eddy simulation (LES) could be used to further capture the behaviour of the flow and to more accurately simulate the eddies. 
If the research were to be developed to involve different spacers, these could also be simulated in order to compare the results and assess the different behaviour of pharmaceutical aerosols, particularly those for asthma, in available spacers. 


\section{Bibliography}

[1] Statistics Canada. Leading Causes of deaths in Canada, 2009.

[2] G Alhegagi and E Matida. Non-symmetrical pmdi deposition measurement in add-on spacers. American Association for Aerosol Research, Oregon Convention Center, Portland, Oregon, USA http://aaarabstracts. com/2010/, 2010.

[3] E Matida and G Alhegagi. Numerical simulation of pmdi aerosol deposition in add-on spacers. American Association for Aerosol Research, Oregon Convention Center, Portland, Oregon, USA http://aaarabstracts. com/2010/, 2010.

[4] SL Fiset. Summer project on the characterisation of the flow of salbutamol sulfate from a pMDI into a volumatic spacer device. Carleton University (Canada), 2012.

[5] MB Dolovich and R Dhand. Aerosol drug delivery: developments in device design and clinical use. The Lancet, 377(9770):1032-1045, 2011.

[6] PW Barry and C O'Callaghan. Inhalational drug delivery from seven different spacer devices. Thorax, 51(8):835-840, 1996.

[7] PW Barry and C O'Callaghan. The optimum size and shape of spacer devices for inhalational therapy. Journal of Aerosol Medicine, 8(3):303-305, 1995.

[8] PW Barry and C O'Callaghan. A comparative analysis of the particle size output of beclomethasone diproprionate, salmeterol xinafoate and fluticasone propionate metered dose inhalers used with the babyhaler, volumatic and aerochamber spacer devices. British journal of clinical pharmacology, 47(4):357-360, 1999.

[9] SI Fox. Fundamentals of human physiology. McGraw-Hill, 2009.

[10] IP Herman. Physics of the human body. Springerverlag Berlin Heidelberg, 2007. 
[11] M Kutz, RS Adrezin, RE Barr, C Batich, RV Bellamkonda, AJ Brammer, TS Buchanan, AM Cook, JM Currie, and et al. Dolan, AM. Standard handbook of biomedical engineering and design. McGraw-Hill, 2003.

[12] H Bisgaard, C O'Callaghan, and GC Smaldone. Drug delivery to the lung, volume 162. Informa Healthcare, 2001.

[13] RA Peura, Y Mendelson, S Kun, and P Pedersen. Biotechnology for biomedical engineers. Engineering in Medicine and Biology Magazine, IEEE, 14(2):199-200, 1995.

[14] JB Grotberg. Respiratory mechanics and gas exchange. 2004.

[15] JB West. Respiratory physiology, the essentials 7th ed. 2005.

[16] AB Lumb. Nunn's Applied Respiratory Physiology. 2005. Oxford: ButterworthHeinemann.

[17] Z Zhang, C Kleinstreuer, and CS Kim. Micro-particle transport and deposition in a human oral airway model. Journal of Aerosol Science, 33(12):1635-1652, 2002.

[18] AJ Hickey. Pharmaceutical inhalation aerosol technology. CRC Press, 2003.

[19] JP Mitchell and MW Nagel. Particle size analysis of aerosols from medicinal inhalers. Kona, 22:32-65, 2004.

[20] WH Finlay. The mechanics of inhaled pharmaceutical aerosols: an introduction. Access Online via Elsevier, 2001.

[21] RB Cole and AD Mackay. Essentials of Respiratory Disease. Churchill Livingstone, 1990. 
[22] The Asthma Society of Canada. Asthma treatment, November 2013. URL http://www . asthma.ca/.

[23] NHS.co.uk. Corticosteroids - NHS choices, 2013. URL http://www.nhs.uk/.

[24] The Lung Association TM. Lung diseases - asthma : The canadian lung association, November 2013. URL http://www.lung.ca/diseases-maladies/ asthma-asthme_e.php.

[25] Novopharm Limited. Novo-salbutamol hfa [product monograph], jan 2011.

[26] D Hartley and D Middlemiss. Absolute configuration of the optical isomers of salbutamol. Journal of medicinal chemistry, 14(9):895-896, 1971.

[27] Daniel N Lapedes. Mcgraw-hill dictionary of scientific and technical terms. 1978.

[28] HDC Smyth. The influence of formulation variables on the performance of alternative propellant-driven metered dose inhalers. Advanced drug delivery reviews, $55(7): 807-828,2003$.

[29] JB Fink, R Dhand, J Grychowski, PJ Fahey, and MJ Tobin. Reconciling in vitro and in vivo measurements of aerosol delivery from a metered-dose inhaler during mechanical ventilation and defining efficiency-enhancing factors. American journal of respiratory and critical care medicine, 159(1):63-68, 1999.

[30] SP Newman. Spacer devices for metered dose inhalers. Clinical pharmacokinetics, 43(6):349-360, 2004.

[31] MR Feddah, KF Brown, EM Gipps, and et al. Davies, NM. In-vitro characterisation of metered dose inhaler versus dry powder inhaler glucocorticoid products: influence of inspiratory flow rates. J Pharm Pharm Sci, 3(3):318-24, 2000. 
[32] BM Crosland. An Experimental Characterisation of the Velocities of the Spray Issued from a Pressurised Metered-dose Inhaler. Carleton University (Canada), 2008.

[33] AMMR Cripps, M Riebe, M Schulze, and R Woodhouse. Pharmaceutical transition to non-cfc pressurized metered dose inhalers. Respiratory medicine, 94: S3-S9, 2000.

[34] DL ROSS and BJ GABRIO. Advances in metered dose inhaler technology with the development of a chlorofluorocarbon-free drug delivery system. Journal of aerosol medicine, 12(3):151-160, 1999.

[35] BJ Lipworth and DJ Clark. Early lung absorption profile of non-cfc salbutamol via small and large volume plastic spacer devices. British journal of clinical pharmacology, 46(1):45-48, 1998.

[36] SP Newman. Principles of metered-dose inhaler design. Respiratory care, 50(9): 1177-1190, 2005.

[37] SP Newman, G Pitcairn, K Steed, A Harrison, and J Nagel. Deposition of fenoterol from pressurized metered dose inhalers containing hydrofluoroalkanes. Journal of allergy and clinical immunology, 104(6):s253-s257, 1999.

[38] OS Usmani, MF Biddiscombe, and PJ Barnes. Regional lung deposition and bronchodilator response as a function of $\beta 2$-agonist particle size. American journal of respiratory and critical care medicine, 172(12):1497-1504, 2005.

[39] SP Newman, AR Clark, N Talaee, and SW Clarke. Pressurised aerosol deposition in the human lung with and without an" open" spacer device. Thorax, 44(9): 706-710, 1989. 
[40] C Kleinstreuer, H Shi, and Z Zhang. Computational analyses of a pressurized metered dose inhaler and a new drug-aerosol targeting methodology. Journal of Aerosol Medicine, 20(3):294-309, 2007.

[41] CA Dunbar. Atomization mechanisms of the pressurized metered dose inhaler. Particulate science and technology, 15(3-4):253-271, 1997.

[42] Watkins AP Dunbar, CA and JF Miller. An experimental investigation of the spray issued from a pMDI using laser diagnostic techniques. Journal of aerosol medicine, 10(4):351-368, 1997.

[43] AR Clark. Metered atomisation for respiratory drug delivery. 1991.

[44] P Rosin and E Rammler. The laws governing the fineness of powdered coal. $J$. Inst. Fuel, 7(31):29-36, 1933.

[45] RF Oliveira, SFCF Teixeira, JCF Teixeira, LF Silva, and H Antunes. pMDI sprays: Theory, experiment and numerical simulation. 2012.

[46] RF Oliveira, SFCF Teixeira, LF Silva, JCF Teixeira, and H Antunes. Development of new spacer device geometry: a CFD study (Part i). Computer Methods in Biomechanics and Biomedical Engineering, 15(8):825-833, 2012.

[47] R Rebelo, R Oliveira, LF Silva, SFCF Teixeira, JCF Teixeira, and H Antunes.

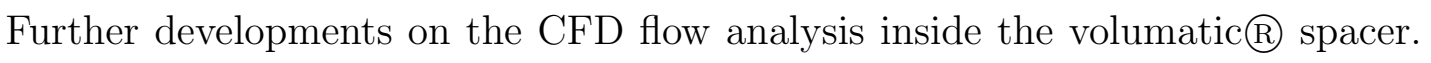
pages $443-451,2010$.

[48] HSM Ali, P York, N Blagden, M Khoubnasabjafari, WE Acree Jr, and A Jouyban. Solubility of salbutamol and salbutamol sulphate in ethanol+ water mixtures at 25 c. Journal of Molecular Liquids, 173:62-65, 2012. 
[49] IHI Habib, MEM Hassouna, and GA Zaki. Simultaneous spectrophotometric determination of salbutamol and bromhexine in tablets. Il Farmaco, 60(3):249$254,2005$.

[50] FR Menter. Two-equation eddy-viscosity turbulence models for engineering applications. AIAA journal, 32(8):1598-1605, 1994.

[51] FR Menter, M Kuntz, and R Langtry. Ten years of industrial experience with the SST turbulence model. Turbulence, heat and mass transfer, 4:625-632, 2003.

[52] O Gryczka, S Heinrich, NG Deen, M van Sint Annaland, JAM Kuipers, M Jacob, and L Mörl. Characterization and CFD-modeling of the hydrodynamics of a prismatic spouted bed apparatus. Chemical Engineering Science, 64(14):3352$3375,2009$.

[53] EA Matida, WH Finlay, M Breuer, and CF Lange. Improving prediction of aerosol deposition in an idealized mouth using large-eddy simulation. Journal of aerosol medicine, 19(3):290-300, 2006.

[54] LS Fan and C Zhu. Principles of gas-solid flows. Cambridge University Press, 2005 .

[55] C Kleinstreuer. Modern Fluid Mechanics: Basic Theory and Selected Applications in Macro-and Micro-fluidics, volume 87. Springer, 2010.

[56] I Kim, S Elghobashi, and WA Sirignano. On the equation for spherical-particle motion: effect of reynolds and acceleration numbers. Journal of Fluid Mechanics, 367(1):221-253, 1998.

[57] MR Maxey and JJ Riley. Equation of motion for a small rigid sphere in a nonuniform flow. Physics of fluids, 26:883, 1983. 
[58] R Mei. Velocity fidelity of flow tracer particles. Experiments in Fluids, 22(1): $1-13,1996$.

[59] T Hayase, JAC Humphrey, and R Greif. A consistently formulated quick scheme for fast and stable convergence using finite-volume iterative calculation procedures. Journal of Computational Physics, 98(1):108-118, 1992.

[60] P Longest and Samir Vinchurkar. Effects of mesh style and grid convergence on particle deposition in bifurcating airway models with comparisons to experimental data. Medical engineering \& physics, 29(3):350-366, 2007.

[61] Joel H Ferziger and Milovan Peric. Further discussion of numerical errors in cfd. International Journal for Numerical Methods in Fluids, 23(12):1263-1274, 1996.

[62] ANSI/ASME standards. Part 1: Measurement Uncertainty, Instruments and Apparatus, 1985.

[63] S Tavoularis. Measurement in fluid mechanics. Cambridge University Press, New York, 2005. 


\section{Appendix A}

\section{Experimental results}

\section{A.1 Deposition results}

Results for the deposition in each section of the spacer for different flow rates, where;

$\mathrm{A}=$ Actuator

$\mathrm{NU}=$ Near upper (quadrant of spacer)

$\mathrm{NL}=$ Near lower (quadrant of spacer)

$\mathrm{DU}=$ Distal upper (quadrant of spacer)

$\mathrm{DL}=$ Distal lower (quadrant of spacer)

Table A.1: Deposition results for $7.5 \mathrm{~L} / \mathrm{min}$

\begin{tabular}{cccccccc} 
& \multicolumn{8}{c}{ Deposition $\mu \mathrm{g}$} \\
\cline { 2 - 8 } Trial & A & NU & NL & DU & DL & Filter & Total deposition \\
\hline \hline 1 & 95.90 & 98.23 & 93.91 & 173.84 & 114.99 & 1420.89 & 1901.86 \\
2 & 133.33 & 100.02 & 115.10 & 96.27 & 149.01 & 1439.05 & 1899.45 \\
3 & 97.65 & 87.63 & 78.14 & 106.55 & 129.88 & 1463.84 & 1866.04 \\
Average & 108.96 & 95.30 & 95.72 & 125.55 & 131.29 & 1411.26 & 1889.12
\end{tabular}


Table A.2: Deposition results for $15 \mathrm{~L} / \mathrm{min}$

\begin{tabular}{cccccccc} 
& \multicolumn{7}{c}{ Deposition $\mu \mathrm{g}$} \\
\cline { 2 - 8 } Trial & A & NU & NL & DU & DL & Filter & Total deposition \\
\hline \hline 1 & 124.79 & 35.92 & 43.74 & 120.53 & 111.68 & 1328.92 & 1640.78 \\
2 & 131.88 & 82.98 & 95.09 & 71.87 & 91.87 & 1337.38 & 1679.19 \\
3 & 90.15 & 95.06 & 69.32 & 62.59 & 94.63 & 1430.39 & 1752.00 \\
Ave & 115.60 & 71.32 & 69.38 & 85.00 & 99.39 & 1365.57 & 1690.66
\end{tabular}

Table A.3: Deposition results for $30 \mathrm{~L} / \mathrm{min}$

\begin{tabular}{cccccccc} 
& \multicolumn{8}{c}{ Deposition $\mu \mathrm{g}$} \\
\cline { 2 - 8 } Trial & A & NU & ND & DU & DL & Filter & Total deposition \\
\hline \hline 1 & 149.30 & 82.10 & 107.10 & 69.67 & 85.97 & 1418.50 & 1763.32 \\
2 & 151.61 & 79.97 & 87.40 & 61.72 & 108.74 & 1638.71 & 1976.54 \\
3 & 111.75 & 60.50 & 62.82 & 64.58 & 87.68 & 1361.70 & 1637.28 \\
Ave & 137.55 & 74.19 & 85.77 & 65.32 & 94.13 & 1472.97 & 1792.38
\end{tabular}

Table A.4: Deposition results for $45 \mathrm{~L} / \mathrm{min}$

\begin{tabular}{cccccccc} 
& \multicolumn{7}{c}{ Deposition $\mu \mathrm{g}$} \\
\cline { 2 - 8 } Trial & A & NU & ND & DU & DL & Filter & Total deposition \\
\hline \hline 1 & 116.81 & 27.55 & 70.81 & 19.72 & 75.06 & 1421.87 & 1731.82 \\
2 & 144.11 & 54.64 & 77.13 & 51.35 & 65.94 & 1388.15 & 1781.33 \\
3 & 122.12 & 63.79 & 75.34 & 54.36 & 81.30 & 1367.63 & 1764.53 \\
4 & 186.49 & 67.70 & 81.27 & 65.00 & 83.03 & 1328.17 & 1811.65 \\
Ave & 142.38 & 53.42 & 76.14 & 47.60 & 76.33 & 1376.46 & 1772.33
\end{tabular}


Table A.5: Deposition results for $60 \mathrm{~L} / \mathrm{min}$

\begin{tabular}{cccccccc} 
& \multicolumn{7}{c}{ Deposition $\mu \mathrm{g}$} \\
\cline { 2 - 8 } Trial & $\mathrm{A}$ & NU & NL & DU & DL & Filter & Total deposition \\
\hline \hline 1 & 174.73 & 48.75 & 107.19 & 33.32 & 83.82 & 1161.45 & 1609.25 \\
2 & 64.94 & 29.70 & 12.61 & 8.45 & 80.98 & 1389.36 & 1514.04 \\
3 & 209.97 & 79.59 & 90.95 & 31.45 & 107.78 & 1505.37 & 2025.07 \\
Ave & 149.87 & 52.68 & 70.25 & 24.40 & 90.86 & 1352.06 & 1716.12
\end{tabular}

\section{A.2 Calibration results}

Table A.6: Absorbance measurements for Beer Lambert calibration curve

\begin{tabular}{ccccccc} 
& \multicolumn{6}{c}{ Concentration, $\mu \mathrm{g} / \mathrm{m}$} \\
\cline { 2 - 7 } & 0.3500 & 0.7000 & 1.4000 & 2.8000 & 5.6000 & 11.2000 \\
\hline \hline Absorbance & 0.0081 & 0.0232 & 0.0340 & 0.0340 & 0.1267 & 0.2480 \\
at & 0.0114 & 0.0239 & 0.0347 & 0.0645 & 0.1283 & 0.2487 \\
$280 \mathrm{~nm}$ & 0.0083 & 0.0227 & 0.0373 & 0.0640 & 0.1291 & 0.2499
\end{tabular}




\section{Appendix B}

\section{Uncertainty in measurements}

Table B.1: Proximal half of spacer

\begin{tabular}{ccccc} 
Flow rate & Deposition Fraction & Bias Limit & Precision limit & Uncertainty \\
\hline \hline $7.5 \mathrm{~L} / \mathrm{min}$ & 0.0956 & 0.0200 & 0.0011 & 0.0385 \\
$15 \mathrm{~L} / \mathrm{min}$ & 0.0779 & 0.0204 & 0.0040 & 0.0668 \\
$30 \mathrm{~L} / \mathrm{min}$ & 0.0829 & 0.0203 & 0.0014 & 0.0429 \\
$45 \mathrm{~L} / \mathrm{min}$ & 0.0731 & 0.0204 & 0.0010 & 0.0372 \\
$60 \mathrm{~L} / \mathrm{min}$ & 0.0716 & 0.0205 & 0.0087 & 0.0954 \\
\hline
\end{tabular}

Table B.2: Distal half of spacer

Flow rate Deposition Fraction Bias Limit Precision limit Uncertainty

\begin{tabular}{llllc}
\hline \hline $7.5 \mathrm{~L} / \mathrm{min}$ & 0.1285 & 0.0194 & 0.0022 & 0.05098 \\
$15 \mathrm{~L} / \mathrm{min}$ & 0.1021 & 0.0199 & 0.0036 & 0.0632 \\
$30 \mathrm{~L} / \mathrm{min}$ & 0.0826 & 0.0203 & 0.0002 & 0.0237 \\
$45 \mathrm{~L} / \mathrm{min}$ & 0.0699 & 0.0205 & 0.0011 & 0.0385 \\
$60 \mathrm{~L} / \mathrm{min}$ & 0.0532 & 0.0208 & 0.0076 & 0.0897 \\
\hline
\end{tabular}


Table B.3: Upper half of spacer

\begin{tabular}{ccccc} 
Flow rate & Deposition Fraction & Bias Limit & Precision limit & Uncertainty \\
\hline \hline $7.5 \mathrm{~L} / \mathrm{min}$ & 0.1105 & 0.0197 & 0.0011 & 0.0383 \\
$15 \mathrm{~L} / \mathrm{min}$ & 0.0865 & 0.0129 & 0.0005 & 0.0264 \\
$30 \mathrm{~L} / \mathrm{min}$ & 0.0723 & 0.0137 & 0.0003 & 0.0215 \\
$45 \mathrm{~L} / \mathrm{min}$ & 0.0570 & 0.0144 & 0.0030 & 0.0562 \\
$60 \mathrm{~L} / \mathrm{min}$ & 0.0449 & 0.0151 & 0.0017 & 0.0438
\end{tabular}

Table B.4: Lower half of spacer

Flow rate Deposition Fraction Bias Limit Precision limit Uncertainty

\begin{tabular}{lcccc}
\hline \hline $7.5 \mathrm{~L} / \mathrm{min}$ & 0.1136 & 0.0183 & 0.0018 & 0.0467 \\
$15 \mathrm{~L} / \mathrm{min}$ & 0.934 & 0.0187 & 0.0006 & 0.0314 \\
$30 \mathrm{~L} / \mathrm{min}$ & 0.0932 & 0.0187 & 0.0005 & 0.0203 \\
$45 \mathrm{~L} / \mathrm{min}$ & 0.0860 & 0.0188 & 0.0001 & 0.0256 \\
$60 \mathrm{~L} / \min$ & 0.0799 & 0.0190 & 0.0197 & 0.1418 \\
\hline
\end{tabular}

